DESY 07-055

HU-EP-07/08

LMU-ASC 79/06

MKPH-T-07-05

\title{
Exploring the structure of the quenched QCD vacuum with overlap fermions
}

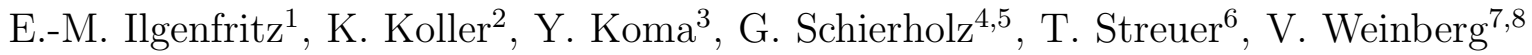 \\ 1 Institut für Physik, Humboldt Universität zu Berlin, 12489 Berlin, Germany \\ 2 Sektion Physik, Universität München, 80333 München, Germany \\ 3 Institut fuer Kernphysik, Johannes-Gutenberg Universität Mainz, 55099 Mainz, Germany \\ 4 Deutsches Elektronen-Synchrotron DESY, 22603 Hamburg, Germany \\ 5 John von Neumann-Institut für Computing NIC, 15738 Zeuthen, Germany \\ 6 Department of Physics and Astronomy, \\ University of Kentucky, Lexington, KY 40506-0055, USA \\ 7 Deutsches Elektronen-Synchrotron DESY, 15738 Zeuthen, Germany \\ 8 Institut für Theoretische Physik, Freie Universität Berlin, 14196 Berlin, Germany \\ - QCDSF Collaboration -
}

\begin{abstract}
Overlap fermions have an exact chiral symmetry on the lattice and are thus an appropriate tool for investigating the chiral and topological structure of the QCD vacuum. We study various chiral and topological aspects of quenched gauge field configurations. This includes the localization and chiral properties of the eigenmodes, the local structure of the ultraviolet filtered field strength tensor, as well as the structure of topological charge fluctuations. We conclude that the vacuum has a multifractal structure.
\end{abstract}




\section{INTRODUCTION}

Overlap fermions [1, 2] possess an exact chiral symmetry on the lattice [3] and realize the Atiyah-Singer index theorem at finite cut-off [4]. Furthermore, they lend themselves to a

local definition of the topological charge density [5]. Altogether, this makes overlap fermions a powerful tool for investigating the chiral and topological structure of the QCD vacuum. In this paper we shall address the vacuum of the quenched theory at zero temperature. Preliminary results have been published in [6, 7, 8, 9]. In a forthcoming paper we shall extend the investigation to dynamical QCD at zero and finite temperature. First results obtained for full QCD in the vicinity of the chiral phase transition have been reported in [10].

It is known for some time [11, 12] that the long distance properties of QCD are well described by the low-lying eigenmodes of the overlap operator. The question of low-mode dominance has been raised earlier in Ref. [13, 14]. In Fig. 1] we compare the full pion propagator with the truncated one spanned only by the 40 lowest eigenmodes.

We see that both propagators tend to each other at distances $\gtrsim 0.5 \mathrm{fm}$. A suitable truncation of the overlap operator thus acts as an infrared filter, which allows us to separate the truly nonperturbative degrees of freedom of the QCD vacuum from the ultraviolet noise. This is in accord with conventional wisdom, namely that chiral symmetry breaking is encoded in the low-lying modes of the Dirac operator and both, chiral symmetry breaking and confinement have the same dynamical origin. To make this more precise, the vacuum structure, seen changing with an increasing number of fermionic modes, is the general theme of this paper. Thus, we shall come back also to the early saturation of the pion propagator in more detail later in this paper.

Earlier lattice investigations of QCD vacuum structure as reviewed in Refs. [15, 16, 17] partly relied on gauge fixing and subsequent projection onto appropriate subgroups of color $S U(3)$. The topics under discussion there are mainly related to the confinement of heavy quarks. In contrast, with the gauge invariant overlap approach, we come closer to the confinement issue of light quarks. As a particular advantage, it establishes a direct link between topological excitations and light quark propagation, which was missing so far. After all, we believe that light fermions are a major element of the low-energy effective action of 


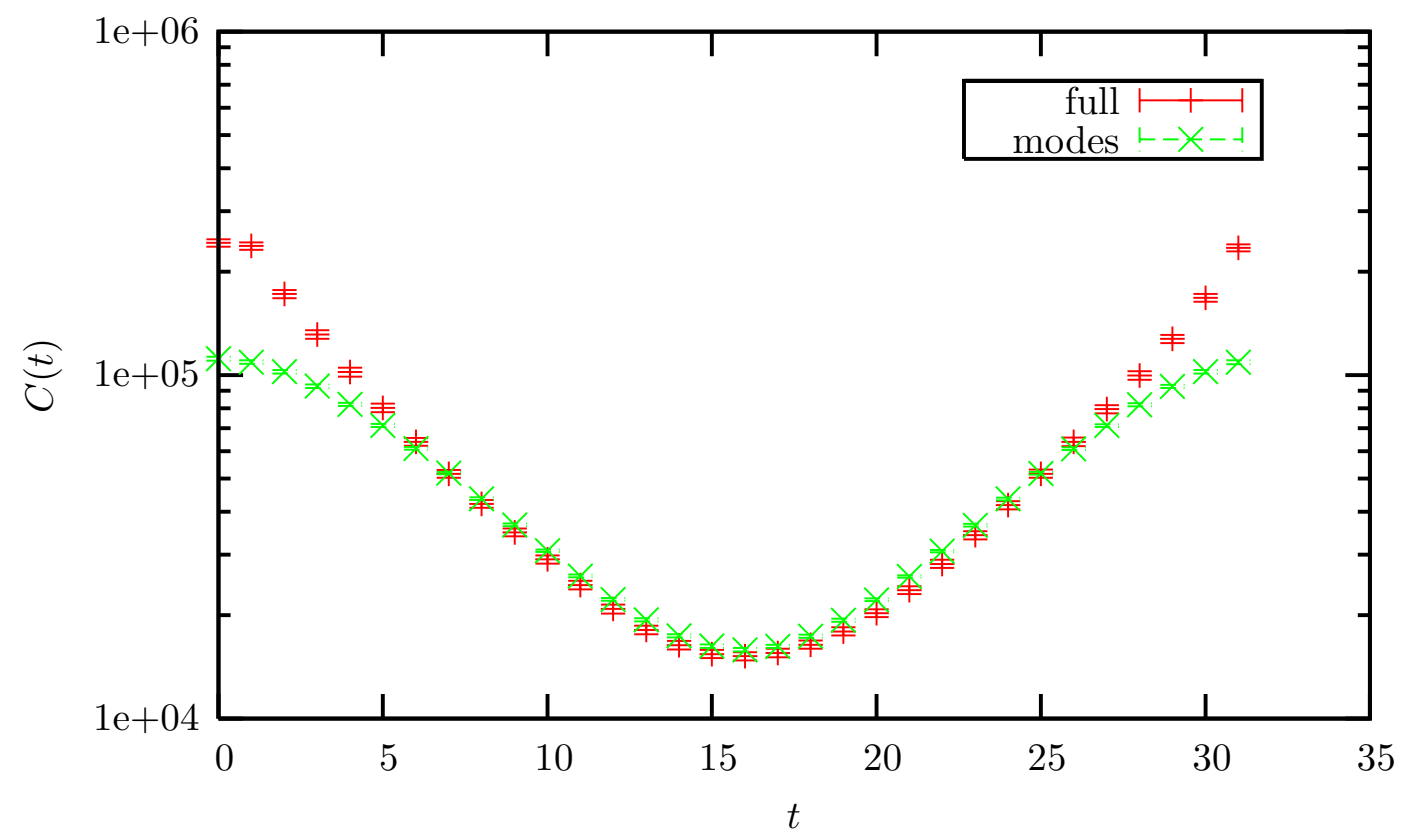

FIG. 1: The full pion propagator compared with the truncated pion propagator constructed as an all-to-all correlator summing over the 40 lowest modes only with a quark mass $\frac{a m}{2 \rho}=0.01$ (see Eq. (12)). This calculation uses 250 configurations on the $16^{3} \times 32$ lattice generated at $\beta=8.45$.

QCD [18].

In the instanton model (as a prototype of other semiclassically motivated models), with the almost-zero-mode band approximation, this link is realized in a very economic way. We summarize these models by saying that they all are based on classical, selfdual or antiselfdual solutions of the Euclidean equations of motion carrying zero modes of definite chirality. While the vacuum fields are formed in these models as superpositions of such extended solutions, in the almost-zero-mode band approximation the spectrum of the lowest quark eigenstates in the complex vacuum is analogously formed by linear combinations of the corresponding zero modes. Examples are the instanton liquid model (for a review see Ref. [19]) where this program has been carried through, or the caloron gas model [20] where this has still to be done. Both models are based on gauge fields piecewise coherent over $O(0.5 \mathrm{fm})$ but with decaying field strength correlations beyond that distance. In order to justify these models, a good deal of lattice vacuum studies has been devoted to instanton and caloron searches.

In the past, cooling [21, 22], underrelaxed cooling [23], restricted improved cooling [24] 
and other smoothing techniques including inverse blocking [25, 26, 27], renormalization group cycling [28], finally rationalized as smearing [29], have been used for this purpose. Since these methods contain a bias towards classical solutions, the results have been taken with certain reservations. In particular, the number density of lumps ("instantons and antiinstantons") was strongly influenced by systematical effects [30]. Only recently, when valence overlap fermions have become a viable option, a local definition of the topological charge density, $q(x) \propto \operatorname{Tr}\left(F_{\mu \nu} \tilde{F}_{\mu \nu}\right)$, in terms of the overlap Dirac operator (see Eq. (18)) became practicable (see e.g. Ref. [31]). Surprisingly, for Monte Carlo configurations this density shows a three-dimensional, laminar and highly singular structure which seems to rule out the instanton and similar pictures.

Easier accessible are mode-truncated, i.e. ultraviolet filtered versions of the topological charge density (see Eq. (19) ). Still with large computational effort, such evaluations of the topological charge density are now superseding cooling or smearing techniques. The latter techniques were necessary to be applied in the past to the gauge field before the topological charge density could be calculated according to a gluonic definition (see for example Ref. [32]). The number of fermionic modes (ultraviolet filtering) now replaces the number of smearing iterations as the control parameter of smoothness. The overlap definition of the topological charge density, if recast [31] into its spectral representation (19), can be evaluated with any desired degree of ultraviolet smearing represented by a cut-off $\lambda_{\text {cut }}$. In this paper the two aspects (the infrared and the ultraviolet one) of the topological structure will be described. Whereas the infrared structure is definitely associated with chiral symmetry breaking, the relation of the surprising divergent structure in the ultraviolet [31, 33, 34] to the phenomenology of confinement, as stressed by [35], is still hypothetic.

The all-scale topological charge density (18), containing fluctuations of all scales, from the lattice spacing $a$ to global structures percolating through the full lattice volume, is calculable [31] directly in terms of the overlap operator. This does not require to know its full spectrum and all eigenmodes. It is calculable, although computationally very demanding, as a local trace due to the form of the Neuberger overlap Dirac operator. In contrast to this, the ultraviolet filtered topological charge density can be quite easily calculated. Moreover, the eigenmodes can be used for many applications [6, 7, 8, 9]. In this paper, for instance, we will elucidate the low-mode dominance of the pion propagator in more detail. 
In the past, selfdual objects have been searched for on the lattice primarily by looking for coherent lumps of topological charge [21, 22, 23, 24, 25, 26, 27, 28, 29] which play a prominent role in models of vacuum structure. When the overlap-based topological charge became calculable, these models were heavily challenged [36, 37]. Early arguments [35] against the instanton dominance of the functional integral have been raised again. The striking new argument against the instanton structure was that the four-dimensional extendedness typical for semiclassical lumps cannot be reconciled with the newly discovered three-dimensional sign-coherent global structures [33] which are infinitely thin in the co-dimension (equal to one [34]). This is the picture that has emerged for the all-scale topological density. All what can be said in defense of instantons etc. is that it is unknown how the effect of quantum fluctuations modifies the topological charge density profile of a classical instanton or caloron which is described by overlap fermions in full agreement with the classical profile.

In this paper, concerned with fully quantum lattice configurations, we will further develop this picture. It is pointed out that the topological charge density, in regions of higher density, possesses lower-dimensional connected structures. In the full four-dimensional landscape we find all substructures, from zero-dimensional peaks over one-dimensional ridges, to twodimensional walls. They are all nested inside the three-dimensional sign coherent structures mentioned above. On the other hand, it will become clear in which sense a model based on a dilute gas of (anti-)selfdual domains can also be supported by an overlap-fermion based analysis. Evidently, for this an ultraviolet filtered version of the overlap-based field strength tensor [38, 39, 40] is necessary, supplemented by the mode-truncated topological charge density [31]. Both can be projected out by a certain small number of true low-lying overlap modes.

This latter point of view is qualitatively attractive because the low-lying fermionic modes are known to encode the most important phenomenological properties of the QCD vacuum relevant for the physics of light hadrons. The case for concentrating on the lowest part of the Dirac spectrum is illustrated by the fact already mentioned that a modest number of eigenmodes is sufficient to reproduce the propagator of the lightest (pseudoscalar) hadrons [11, 12, 13, 14] (see also Fig. 11). The lowest-mode dominance allows for a systematic improvement of the propagator by the so-called low-mode averaging method [41, 42, 43]. In this and a following paper this will be investigated more carefully. 
Recently, the localization properties [6, 44, 45, 46, 47, 48], and the effective dimension of the lower eigenmodes, and their local chiral properties [36, 37, 49, 50] have received strong interest. The results partly contradict each other. Concerning the localization the situation has been reviewed in Ref. [51]. There, de Forcrand points out the basic difference between $S U(2)$ fields simulated with Wilson action, when scale $a$ dislocations have too low action [52] to compensate for their entropy $\sim \log a^{-4}$, and $S U(3)$ gauge fields simulated with Lüscher-Weisz action with dislocations suppressed. The general motivation behind this high attention for issues like localization and (fractal) dimensionality was the hope [53, 54] that the lowest modes are bound to certain singularities of the gauge field that in turn would explain confinement. Here, "singular" is understood in a sense opposite to semiclassical lumps, just in the spirit of Witten's [35] criticism of instantons. This is discussed from a more recent point of view in Refs. [55, 56]. Uncorrelated instantons, indeed, are found unrelated to confinement. It should be mentioned in passing, however, that other semiclassical configurations with non-trivial asymptotic holonomy can be related to confinement, as was recently demonstrated in Ref. [20].

Another source of inspiration was the hypothetical analogy drawn between the finitetemperature chiral transition to the Anderson transition (metal insulator transition) [57, 58, 59] in condensed matter physics. As precursor of the chiral symmetry restoring transition, before the spectral gap opens, the spectrum in the gap region is expected to become critical, exposing both spectral criticality and multifractality of modes. We will come back to these aspects in a forthcoming publication dealing with finite temperature full QCD. In the present case of quenched QCD the whole low-lying spectrum seems to be critical and multifractal.

This paper is organized as follows. In Section II the basic tools and settings of the quenched simulation and for handling the eigenmodes of the overlap operator are explained. In Section III the properties of individual modes are discussed. This begins with the distribution of the topological charges $Q$ (given by the number and chirality of zero modes) and the spectral density of non-zero modes. From the latter, the chiral condensate is extracted by fitting our finite-volume data to quenched chiral perturbation theory [60]. Then the localization behavior, the dimensionality and the local chiral properties (chirality $X(x)$ ) of individual modes are discussed. In Section IV we describe our experience with the topological charge density defined [5] through the overlap Dirac operator and its ultraviolet 
filtered (mode-truncated) variant. The existence of a three-dimensional, singular and signcoherent global structure revealed by the all-scale topological charge density, first pointed out in Ref. [33], is confirmed and described in more detail. The negativity of the two-point function of the topological charge density [6, 61] is found to be realized for the all-scale topological charge density for high enough $\beta$, i.e. at lattice spacing $a \lesssim 0.1 \mathrm{fm}$. In the same Section [V] we also discuss the topological structure in terms of what we call $q$-clusters. The multiplicity, size, distance and the percolation behavior of $q$-clusters is compared for various levels of ultraviolet filtering $\left(\lambda_{\text {cut }}\right)$ and for the all-scale topological charge density. With an appropriate $\lambda_{\text {cut }}$ the $q$-cluster structure of the mode-truncated density $q_{\lambda_{\text {cut }}}(x)$ can be made agreeing with the $R$-cluster structure defined later in Section V. Finally, estimating the fractal dimension of $q$-clusters related to the all-scale topological charge density at various threshold values $q_{\text {cut }}$, we find that the three-dimensional structure of sign-coherent topological charge is supplemented by nested, lower-dimensional substructures at higher density $|q(x)|$. Obviously these features have no analog for the mode-truncated topological charge density. In Section $\mathrm{V}$ an ultraviolet filtering technique for the field strength tensor is introduced. It was inspired by a similar work by Gattringer [38]. The representation of the field strength tensor in terms of the overlap Dirac operator was later discussed by Liu et al. [39, 40]. An infrared field strength can be obtained that allows one to assign at each point in space-time the degree $R(x)$ of selfduality or antiselfduality. Connected clusters (called $R$-clusters) are found such that in the interior (anti-)selfduality is approximately satisfied. These clusters start to percolate and form a dilute network if slight deviations from (anti)selfduality are tolerated. Thus, locally perfectly selfdual and antiselfdual vacuum fields

are embedded in a vacuum that is globally neither selfdual nor antiselfdual. In Section VI we summarize our findings and discuss from this point of view the saturation of the pion propagator. We draw conclusions and point out routes for further research.

\section{BASIC TOOLS}

\section{A. Lattice ensembles}

Topological studies using the Wilson one-plaquette gauge field action suffer from dislocations [62] and should be treated with caution. For this paper the Lüscher-Weisz gauge 
field action [63] is used which is known to suppress dislocations and to greatly reduce the number of unphysical zero modes of the Wilson-Dirac operator. The Lüscher-Weisz gauge field action is given by

$$
\begin{aligned}
& S[U]=\beta \sum_{\text {plaquette }} \frac{1}{3} \operatorname{Re} \operatorname{Tr}\left(1-U_{\text {plaquette }}\right) \\
& +\beta_{R} \sum_{\text {rectangle }} \frac{1}{3} \operatorname{Re} \operatorname{Tr}\left(1-U_{\text {rectangle }}\right) \\
& +\beta_{P} \sum_{\text {parallelogram }} \frac{1}{3} \operatorname{Re} \operatorname{Tr}\left(1-U_{\text {parallelogram }}\right)
\end{aligned}
$$

in terms of the standard plaquette, the planar rectangle and the parallelogram loop terms. The latter are closed along the diagonally opposite parallel links on the surface of a 3D cube. The coefficients $\beta_{R}$ and $\beta_{P}$ are taken from tadpole improved perturbation theory [64, 65, 66, 67]

$$
\begin{aligned}
& \beta_{R}=-\frac{\beta}{20 u_{0}^{2}}(1+0.4805 \alpha), \\
& \beta_{P}=-\frac{\beta}{u_{0}^{2}} 0.03325 \alpha
\end{aligned}
$$

where

$$
u_{0}=\left(\frac{1}{3}\left\langle\operatorname{Re} \operatorname{Tr} U_{\text {plaquette }}\right\rangle\right)^{1 / 4} \quad, \quad \alpha=-\frac{\log \left(u_{0}^{4}\right)}{3.06839} .
$$

The couplings $\beta_{R}$ and $\beta_{P}$ have been selfconsistently determined through the calculation of the average plaquette in Ref. [68] for a set of $\beta$ values. We can write $\beta=c_{0} \frac{6}{g^{2}}, \beta_{R}=c_{1} \frac{6}{g^{2}}$ and $\beta_{P}=c_{2} \frac{6}{g^{2}}$ with $c_{0}+8 c_{1}+8 c_{2}=1$, which fixes the relation between $\beta$ and the bare coupling $g^{2}$.

To investigate the volume dependence of our data we have simulated at three different volumes at fixed coupling $\beta=8.45$. To explore the $a$ dependence of the results also a $12^{3} \times 24$ lattice at $\beta=8.10$ has been employed with approximately the same physical volume as the $16^{3} \times 32$ lattice at $\beta=8.45$. Finally, a large ensemble of rather coarse configurations on a $16^{3} \times 32$ lattice at $\beta=8.00$ became available in the QCDSF collaboration. A first physics analysis on this basis has been presented in Ref. [43]. The physical volume of this ensemble almost equals the biggest physical volume studied on $24^{3} \times 48$ at $\beta=8.45$.

In Table I the statistics of lattices used in our investigation is listed. The lattice spacing for $\beta=8.00$ and $\beta=8.45$ was determined in [43], where we used the pion decay constant of 


\begin{tabular}{|c|c|c|c|c|c|c|c|}
\hline$\beta$ & $a[\mathrm{fm}]$ & $L_{s}^{3} \times L_{t}$ & $a L_{s}[\mathrm{fm}]$ & $V\left[\mathrm{fm}^{4}\right]$ & $\chi_{\text {top }}$ & \# of config. & \# of modes \\
\hline \hline 8.45 & $0.105(2)$ & $12^{3} \times 24$ & 1.3 & 5 & {$[167(3) \mathrm{MeV}]^{4}$} & 437 & $O(50)$ \\
8.45 & $0.105(2)$ & $16^{3} \times 32$ & 1.7 & 16 & {$[169(3) \mathrm{MeV}]^{4}$} & 400 & $O(150)$ \\
8.45 & $0.105(2)$ & $24^{3} \times 48$ & 2.5 & 81 & {$[168(4) \mathrm{MeV}]^{4}$} & 250 & $O(150)$ \\
\hline 8.10 & $0.142(2)$ & $12^{3} \times 24$ & 1.7 & 15 & {$[171(1) \mathrm{MeV}]^{4}$} & 251 & $O(150)$ \\
\hline 8.00 & $0.157(3)$ & $16^{3} \times 32$ & 2.5 & 74 & {$[172(4) \mathrm{MeV}]^{4}$} & 2156 & $O(170)$ \\
\hline
\end{tabular}

TABLE I: Details of the quenched ensembles used in this study: couplings $\beta$, lattice spacings $a$ determined from the pion decay constant, lattice sizes $\left(L_{s}\right.$ and $\left.L_{t}\right)$, physical lattice sizes $\left(a L_{s}\right.$ and the volume $V$ ), topological susceptibilities $\chi_{\text {top }}$, the statistics of configurations and available overlap fermion modes.

$f_{\pi}=92.4 \mathrm{MeV}$ to set the scale. The value of the lattice spacing at $\beta=8.10$ is interpolated by fitting our measured pion decay constants at $\beta=8.00$ and $\beta=8.45$ to equation (6) of [68].

It is in this scale that the spatial linear extent $a L_{s}$ of the lattices, the $4 \mathrm{D}$ volumes $V$ and the topological susceptibilities $\chi_{\text {top }}$ are obtained. The topological susceptibilities will be discussed in Section

\section{B. Implementation of Neuberger overlap fermions}

Overlap fermions [1, 2] have an exact chiral symmetry on the lattice [3] and provide the cleanest known theoretical description of lattice fermions. Their implementation of chiral symmetry and the possibility to exactly define the index theorem on the lattice at finite lattice spacing allow one to investigate the relationship of topological properties of gauge fields and the dynamics of fermions. A further advantage of overlap fermions, in contrast to Wilson fermions, is that they are automatically $O(a)$ improved [69]. 
The massless Neuberger overlap operator is defined by

$$
D(0)=\frac{\rho}{a}\left(1+\frac{D_{W}}{\sqrt{D_{W}^{\dagger} D_{W}}}\right), \quad D_{W}=M-\frac{\rho}{a},
$$

where we use the Wilson-Dirac operator $D_{W}$ as input. $M$ is Wilson's hopping term with $r=1$. The negative mass parameter $\rho$ is chosen to be 1.4 , which represents a reasonable compromise between the physical requirement of good locality properties [43, 70] of the overlap operator and a performance requirement, demanding a small condition number of $H_{W}^{2}$, where $H_{W}=\gamma_{5} D_{W}$ is the Hermitean Wilson-Dirac operator. In Fig. 2 the effective range of $D(0)$, represented by the decay of

$$
F(r)=\left\langle\left\langle\left.\max _{x}|D(0 ; x, y)|\right|_{|x-y|=r}\right\rangle_{y}\right\rangle_{U}
$$

with respect to the Euclidean distance

$$
|x|=\left(\sum_{\mu=1}^{4} x_{\mu}^{2}\right)^{\frac{1}{2}}
$$

is shown, as obtained on a $16^{3} \times 32$ lattice at $\beta=8.45$ for $\rho=1.4$,

Asymptotically, $F(r) \propto \exp (-\mu r / a)$, where $\mu$ depends on $\rho$ (and a priori also on the roughness of the configurations, i.e. $\beta$ ). It turns out, however, that the fitted slope $\mu=$ $1.11(1)$ is practically independent of $\beta$. Thus, the overlap Dirac operator is not ultralocal. It has a range that shrinks together with the lattice spacing towards the continuum limit. This is sufficient for any Dirac operator $D$ to be called local.

To compute the sign function

$$
\operatorname{sgn}\left(D_{W}\right)=\frac{D_{W}}{\sqrt{D_{W}^{\dagger} D_{W}}} \equiv \gamma_{5} \operatorname{sgn}\left(H_{W}\right), \quad H_{W}=\gamma_{5} D_{W}
$$

we write

$$
\operatorname{sgn}\left(H_{W}\right)=\sum_{i=1}^{N} \operatorname{sgn}\left(\mu_{i}\right) \chi_{i} \chi_{i}^{\dagger}+P_{\perp}^{N} \operatorname{sgn}\left(H_{W}\right) .
$$

The first part is exactly handled in the subspace of eigenvectors $\chi_{i}$ of $H_{W}$, the rest acting in the orthogonal subspace is approximated by a minmax polynomial [71] satisfying

$$
\left|P_{\operatorname{minmax}}(x)-\frac{1}{\sqrt{x}}\right|<\epsilon \quad \text { for } \quad \mu_{N+1}^{2}<x<\mu_{\max }^{2}
$$




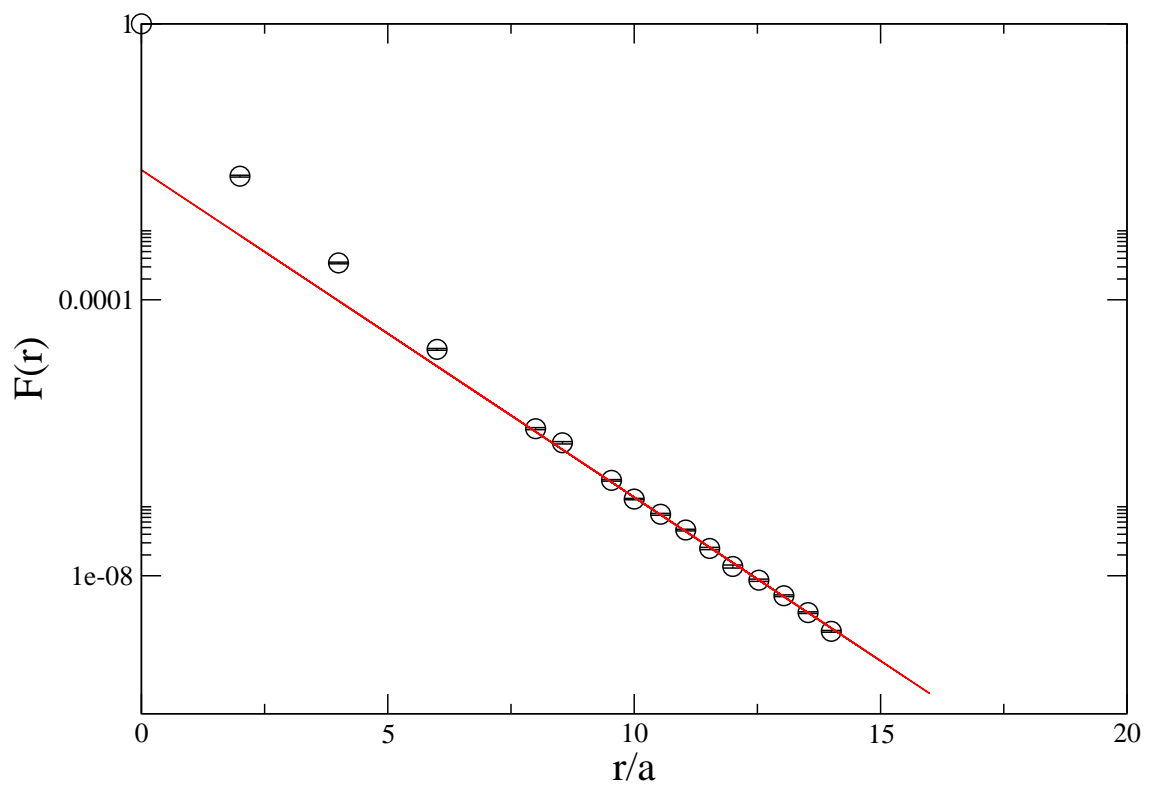

FIG. 2: The effective range function $F(r)$ as a function of $r / a$ on the $16^{3} \times 32$ lattice at $\beta=8.45$ for $\rho=1.4$, together with an exponential fit.

such that

$$
\operatorname{sgn}\left(H_{W}\right)=\sum_{i}^{N} \operatorname{sgn}\left(\mu_{i}\right) \chi_{i} \chi_{i}^{\dagger}+P_{\perp}^{N} H_{W} P_{\operatorname{minmax}}\left(H_{W}^{2}\right) .
$$

The lowest $N=O(50)$ eigenvalues $\mu_{i}$ of $H_{W}$ and the corresponding eigenvectors $\chi_{i}$ have to be computed. The degree of the polynomial $P_{\operatorname{minmax}}$ depends on $\epsilon$ and is proportional to the condition number $\kappa=\mu_{\max }^{2} / \mu_{N+1}^{2}$ of $H_{W}^{2}$ in the orthogonal subspace spanned by the modes $\left\{\chi_{i} \mid\left(1-P_{\perp}^{N}\right) \chi_{i}=0\right\}$. For configurations generated with the Lüscher-Weisz action, the condition number of $H_{W}$ is essentially smaller in comparison with the Wilson action and only slightly depends on $\rho$.

$D(0)$ as described above is a massless Dirac operator. The massive overlap Dirac operator is easily obtained from the massless one, as [69]

$$
D(m)=\left(1-\frac{a m}{2 \rho}\right) D(0)+m
$$

Later on, in our spectral analysis we use the improved massless Neuberger operator $[72$, 
73],

$$
D^{\mathrm{imp}}(0)=\left(1-\frac{a}{2 \rho} D(0)\right)^{-1} D(0) .
$$

The resulting eigenvalues $\lambda_{\text {imp }}$ of $D^{\mathrm{imp}}(0)$ are the stereographical projection of the eigenvalues $\lambda_{N}$ of $D(0)$ from the Ginsparg-Wilson circle to the imaginary axis. The non-zero eigenvalues appear in pairs $\lambda_{\text {imp }} \equiv \pm i \lambda$. In terms of these imaginary parts $\lambda$, the spectral density and the characteristic properties of the eigenmodes are discussed. Also any mode cut-off $\lambda_{\text {cut }}$ refers to this $\lambda$.

Finally, the improved massive overlap Dirac operator [43], which is used for example for the pion propagator in this paper, is

$$
D^{\mathrm{imp}}(m)=D^{\mathrm{imp}}(0)+m .
$$

\section{Eigenmodes of the Neuberger operator}

In principle, the emerging Neuberger overlap operator $D(0)$ could have $n_{-}$plus $n_{+}$exact zero modes, $D(0) \psi_{n}=0$, with $n_{-}\left(n_{+}\right)$being the number of modes with negative (positive) chirality, $\gamma_{5} \psi_{n}=-\psi_{n}\left(\gamma_{5} \psi_{n}=+\psi_{n}\right)$. The index of $D(0)$ agrees with the topological charge, $Q=n_{-}-n_{+}$. In practice, however, there are always only negative or positive chirality eigenmodes for a given lattice configuration. The non-zero eigenvalues $\lambda_{N}$ and their nonchiral eigenvectors with $D(0) \psi_{\lambda_{N}}=\lambda_{N} \psi_{\lambda_{N}}$ appear in complex conjugate pairs $\lambda_{N}$ and $\lambda_{N}^{*}$ and satisfy $\sum_{x}\left(\psi_{\lambda_{N}}^{\dagger}(x), \gamma_{5} \psi_{\lambda_{N}}(x)\right)=0$.

Locally, the eigenmodes $\psi_{\lambda}$ of the Neuberger operator can be characterized by the scalar density

$$
p_{\lambda}(x)=\left|\psi_{\lambda}(x)\right|^{2}=\sum_{\sigma, c} \psi_{\lambda}^{\sigma c *}(x) \psi_{\lambda}^{\sigma c}(x), \quad \sum_{x} p_{\lambda}(x)=1,
$$

where $\sigma$ and $c$ denote spinor and color indices.

Besides the scalar density, another important density needed is the pseudoscalar density

$$
p_{\lambda 5}(x)=\sum_{\sigma, \sigma^{\prime}, c} \psi_{\lambda}^{\sigma c *}(x) \gamma_{5}^{\sigma \sigma^{\prime}} \psi_{\lambda}^{\sigma^{\prime} c}(x), \quad \sum_{x} p_{\lambda 5}(x)= \pm 1 \quad \text { or } \quad 0
$$

for chiral or non-chiral modes, respectively. While the zero modes are globally chiral, the 
non-chiral non-zero modes may still have a local chirality,

$$
p_{\lambda \pm}(x)=\sum_{\sigma, c} \psi_{\lambda}^{\sigma * *}(x) P_{ \pm}^{\sigma \sigma^{\prime}} \psi_{\lambda}^{\sigma^{\prime} c}(x)
$$

with the projectors $P_{ \pm}=\left(1 \pm \gamma_{5}\right) / 2$ onto positive and negative chirality.

\section{Overlap definition of the all-scale and the mode-truncated topological density}

As for any $\gamma_{5}$-Hermitean Dirac operator satisfying the Ginsparg-Wilson relation, the topological charge density for the Neuberger operator $D(0)$ can be expressed as [4]:

$$
q(x)=-\operatorname{tr}\left[\gamma_{5}\left(1-\frac{a}{2} D(0 ; x, x)\right)\right], \quad Q=\sum_{x} q(x)
$$

where the trace tr is taken over color and spinor indices.

To compute the topological charge density, we use two different approaches [31, 33]. In the first approach, the trace of the overlap operator is directly evaluated according to Eq. (18). This is computationally very demanding and is therefore performed on only $53(5)$ configurations in the case of a $12^{3} \times 24$ lattice $\left(16^{3} \times 32\right.$ lattice, respectively) at $\beta=8.45$. The all-scale density $q(x)$ computed in this way includes charge fluctuations at all scales. For this density, interesting anisotropic, global structures have been detected and discussed by Horvath et al. [34]. In this paper we will give more details on the multifractal properties of $q(x)$.

The second technique involves the computation of the topological charge density based only on the low-lying modes of the overlap Dirac operator. This approach is a gauge invariant filtering that leaves the lattice configuration unchanged (as well as the effective lattice spacing as the physical scale). Using the spectral representation of the Dirac operator, the truncated eigenmode expansion of the topological charge density reads

$$
q_{\lambda_{\mathrm{cut}}}(x)=-\sum_{|\lambda|<\lambda_{\mathrm{cut}}}\left(1-\frac{\lambda}{2}\right) p_{\lambda 5}(x),
$$

with $p_{\lambda 5}(x)$ defined in Eq. (16). Truncating the expansion at $\lambda_{\text {cut }}$ acts like an ultraviolet filter by removing the short-distance fluctuations from $q(x)$. We will study in more detail how the properties of this density depend on the choice of $\lambda_{\text {cut }}$. With a suitable cut-off 
the clusters of this density will coincide with the $R$-clusters of (anti-)selfdual domains to be introduced in Section VC. Note that the total topological charge $Q=\sum_{x} q_{\lambda_{\text {cut }}}(x)$ is not affected by the choice of $\lambda_{\text {cut }}$, the level of truncation.

\section{E. Cluster analysis}

In this paper we will describe the properties of different densities in terms of their cluster properties. This is the appropriate place to describe the cluster algorithm in general terms. As an example we discuss this for the topological charge density $q(x)$.

1. The cluster analysis first requires tagging the lattice sites that will form the clusters. In the present case these are all sites with $|q(x)|>q_{\text {cut }}$.

2. Next these tagged sites are assigned to a set of link-connected clusters. Two sites $x$ and $y$ are said to be link-connected if they are immediate neighbors on the lattice connected by a link, $x=y \pm \hat{\mu}$. Two link-connected and tagged sites $x$ and $y$ then belong to the same cluster unless $q(x)$ and $q(y)$ have opposite sign. Obviously, the latter veto is ineffective in the case of a positive density like the scalar density of eigenmodes.

3. Cluster percolation is defined by the cluster correlation function which is given as the following ensemble average:

$$
f(r)=\frac{\sum_{x, y}\left\langle\sum_{c} \Theta_{c}(x) \Theta_{c}(y)\right\rangle \delta(r-|x-y|)}{\sum_{x, y} \delta(r-|x-y|)}
$$

where $\Theta_{c}(x)$ is the characteristic function of a cluster $c$, i.e. $\Theta_{c}(x)=1$ if $x \in c$ and $\Theta_{c}(x)=0$ otherwise. In the definition (20) $r$ is the Euclidean distance. If $r_{\max }$ is the largest distance possible on the periodic lattice, we call the value $f\left(r_{\max }\right)$ "connectivity". The onset of percolation is defined as the value of the cluster-defining quantity, i.e. of the cut-off $q_{\text {cut }}=q_{\text {perc }}$, such that the connectivity $f\left(r_{\max }\right) \neq 0$ for $q_{\text {cut }}<q_{\text {perc }}$.

4. An important quantity describing the system of clusters is the Euclidean distance between two clusters, for example the two biggest clusters. The distance $d\left(c, c^{\prime}\right)$ between 
two clusters labeled $c$ and $c^{\prime}$ can be defined as the maximum over sites $x \in c$ of the minimal Euclidean distance between $x$ and any site $y \in c^{\prime}$,

$$
d\left(c, c^{\prime}\right)=\max _{x \in c}\left(\min _{y \in c^{\prime}}(|x-y|)\right) .
$$

5. Other quantities describing the system of clusters in a given configuration are the fraction of occupied volume, the fraction of the largest cluster to the total occupied volume, the size and charge distributions of clusters and the total multiplicity of clusters.

We will use this terminology when we shall discuss qualitative features of certain densities.

\section{F. Random walkers}

For the characterization of a $q$-cluster with respect to its fractal dimension, we use the random walker method briefly described below. We consider a collection of independent random walkers starting from the maximum $q_{\max }$ of the topological charge density inside a cluster among a collection of clusters defined by a cut-off $q_{\text {cut }}$. Depending on the ratio $q_{\text {cut }} / q_{\text {max }}$, the cluster is more or less extended. The cluster determines where the random walkers are permitted to go. The random walk is defined by the condition that at each time step $\tau \rightarrow \tau+1$ the walker is required to jump with equal probability to one of the neighboring sites that also belong to the cluster. If $q_{\text {cut }} / q_{\max }$ is large, the cluster is small, such that the random walk soon arrives at a stationary regime. If $q_{\text {cut }} / q_{\max }$ is smaller, the cluster is extended in one or more directions, the number of which one want to explore. The fractal dimension $d^{*}$ which is open for the random walkers will then be reflected by the return probability to the starting point,

$$
P(\overrightarrow{0}, \tau)=(2 \pi \tau)^{-\frac{d^{*}}{2}}
$$

following a power-like decay with the number $\tau$ of time-steps. This can be defined for one cluster or as average over all clusters.

Also the distribution of all walkers in one cluster at a given time-step can be useful to characterize the shape of the cluster. This method is applicable to fractal clusters of any kind, for example also those of the scalar density of the individual fermionic modes. 


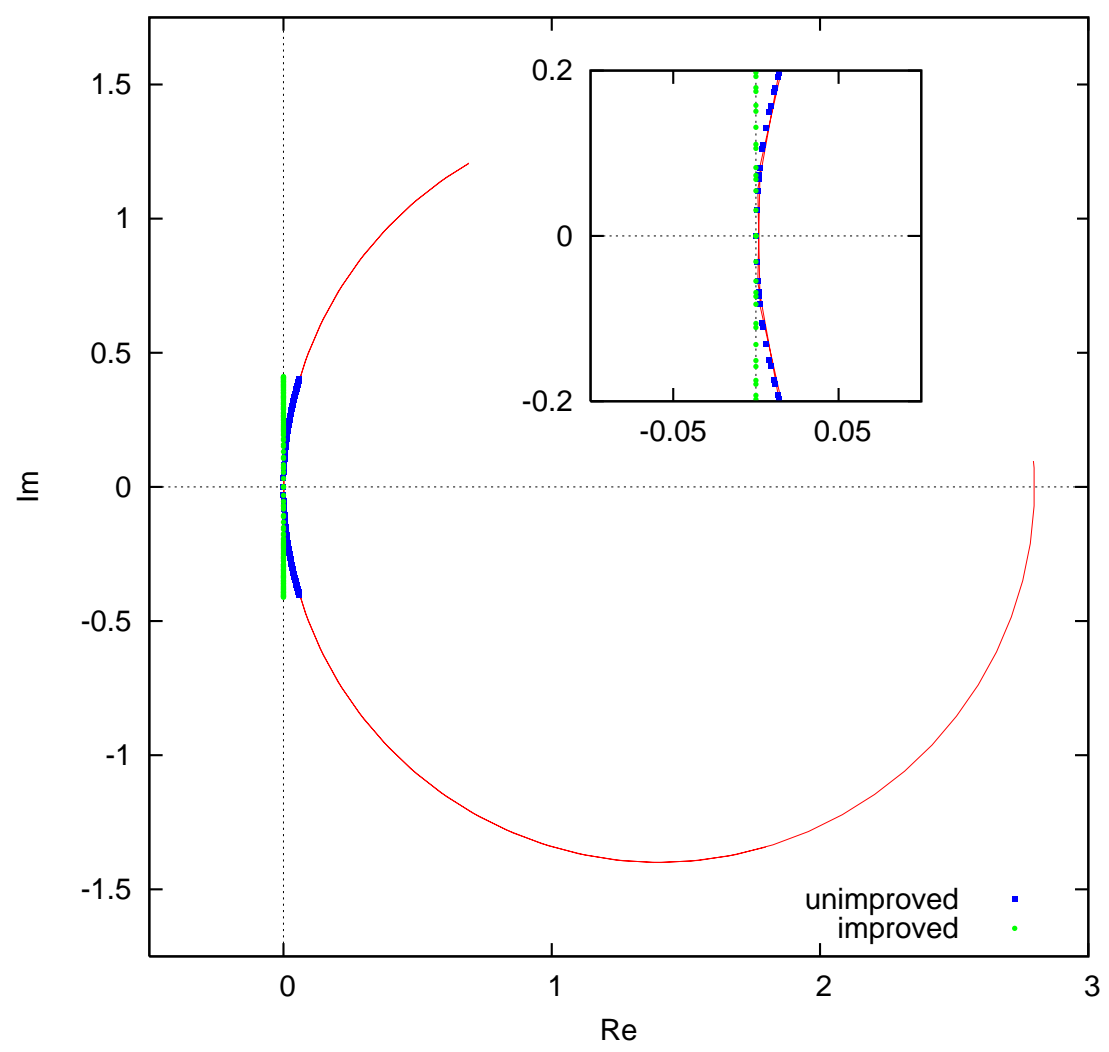

FIG. 3: The Ginsparg-Wilson circle and the analyzed part of the unimproved and improved spectrum for a $Q=3$ configuration on the $16^{3} \times 32$ lattice generated at $\beta=8.45$. The insert shows the lowest part of the spectrum magnified. The zero eigenvalue is threefold degenerate.

\section{SPECTRAL RESULTS BASED ON THE LOWEST O(150) EIGENMODES}

\section{A. The Ginsparg-Wilson circle and the topological charge from the index of $D(0)$}

Using the above construction of the Neuberger overlap operator, we perform its diagonalization by a variant of the implicitly restarted Arnoldi algorithm. Mostly an amount of $O(150)$ eigenvalues and eigenvectors per configuration has been computed and stored. The complex-valued eigenvalues $\lambda_{N}$ are located on the Ginsparg-Wilson circle of radius $\rho$ around the point $(\rho, 0)$ in the complex plane. This is shown in Fig. 3 .

In Fig. 4 (a) to (e) the distribution of topological charge $Q=n_{-}-n_{+}$over the various ensembles is shown as determined from the number and chirality of zero modes of the lattice configurations. As has been said, we have never found zero modes with different chirality 
simultaneously in the same configuration.

From the distribution of topological charges shown in Figs. 4 the topological susceptibilities

$$
\chi_{\mathrm{top}}=\frac{\left\langle Q^{2}\right\rangle}{V}
$$

have been evaluated. They are also given in Table I. It is remarkable that all physical lattice

sizes realized with $\beta=8.45$ are big enough to get the topological susceptibility equal within error bars. At the same time, the topological susceptibilities of the coarser lattices are only slightly larger, in accordance with the fact that other quantities are found to scale well with $\beta$ [43]. The topological susceptibilities at $\beta=8.1$ and $\beta=8.0$ are equal to each other within error bars although the physical volumes differ by a factor five. All results are smaller than the topological susceptibility of quenched $S U(3)$ gauge theory reported in Ref. [74] to be $(191 \pm 5 \mathrm{MeV})^{4}$. This value was given using $f_{K}=160(2) \mathrm{MeV}$ to set the scale.

\section{B. Spectral density of non-zero modes}

The spontaneous breaking of chiral symmetry by the dynamical creation of a nonvanishing chiral condensate $\langle\bar{\Psi} \Psi\rangle$ is related to the spectral density $\rho(\lambda)$ of non-zero modes of the Dirac operator near zero by the Banks-Casher relation [75] $\langle\bar{\Psi} \Psi\rangle=-\pi \rho(0)$.

The spectral density of the continuous modes at finite volume is formally given by

$$
\rho(\lambda, V)=\frac{1}{V}\left\langle\sum_{\bar{\lambda}} \delta(\lambda-\bar{\lambda})\right\rangle,
$$

where the sum extends only over positive (non-zero) values of $\bar{\lambda}=\operatorname{Im} \lambda_{\text {imp }}$ of the eigenvalues of the improved massless overlap operator $D^{\mathrm{imp}}(0)$. The average $\langle\ldots\rangle$ is taken over the ensemble of gauge field configurations.

In the finite volume and for small eigenvalues the spectral density can be computed from the chiral low-energy effective theory. For $\lambda<E_{T}, E_{T}$ being the Thouless energy $E_{T} \approx$ $f_{\pi}^{2} / \Sigma \sqrt{V}$, the low-energy effective partition function is dominated by the zero momentum modes, and the zero-momentum approximation of the chiral low-energy effective theory is equivalent to chiral random matrix theory.

In random matrix theory the spectral density is given as 


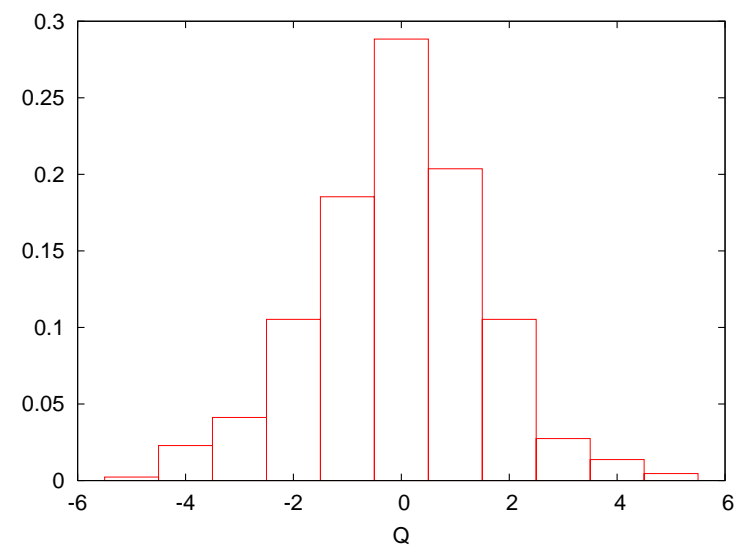

(a)

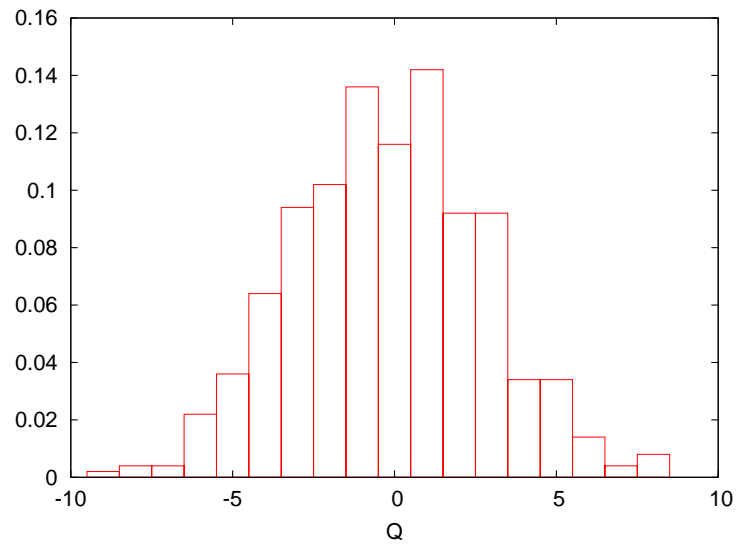

(b)

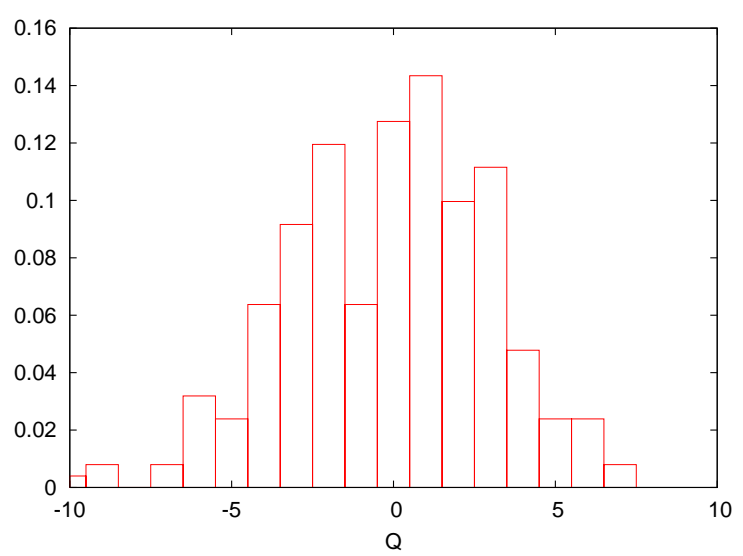

(d)

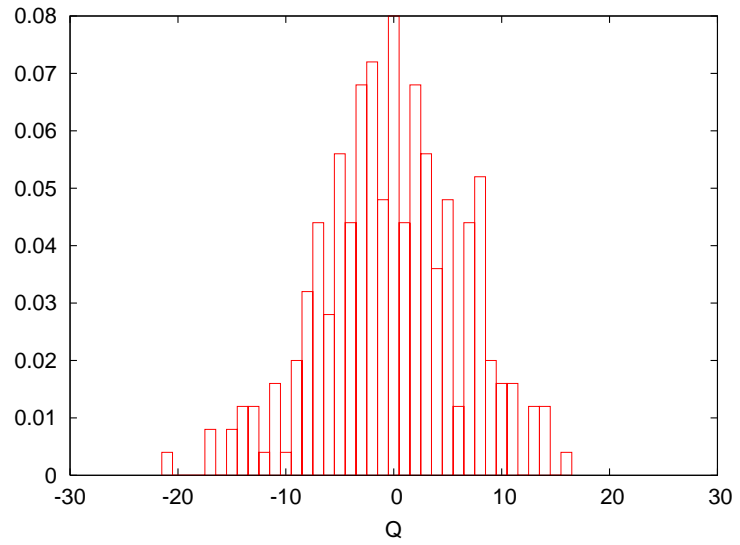

(c)

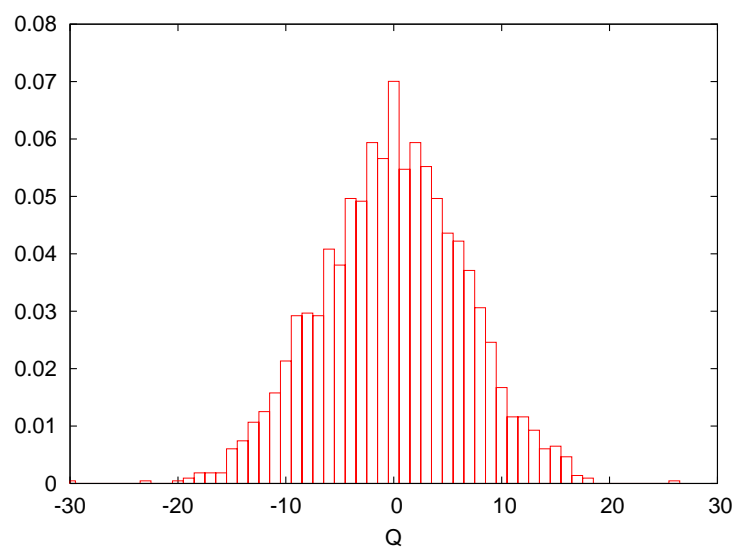

(e)

FIG. 4: The normalized distributions of topological charge $Q$, in subfigures ordered according to descending $\beta$, for (a) $12^{3} \times 24$ at $\beta=8.45$, (b) $16^{3} \times 32$ at $\beta=8.45$, (c) $24^{3} \times 48$ at $\beta=8.45$, (d) $12^{3} \times 24$ at $\beta=8.10$, (e) $16^{3} \times 32$ at $\beta=8.00$. Note that the physical volumes corresponding to (b) and (d) are roughly equal, and similarly for (c) and (e). 


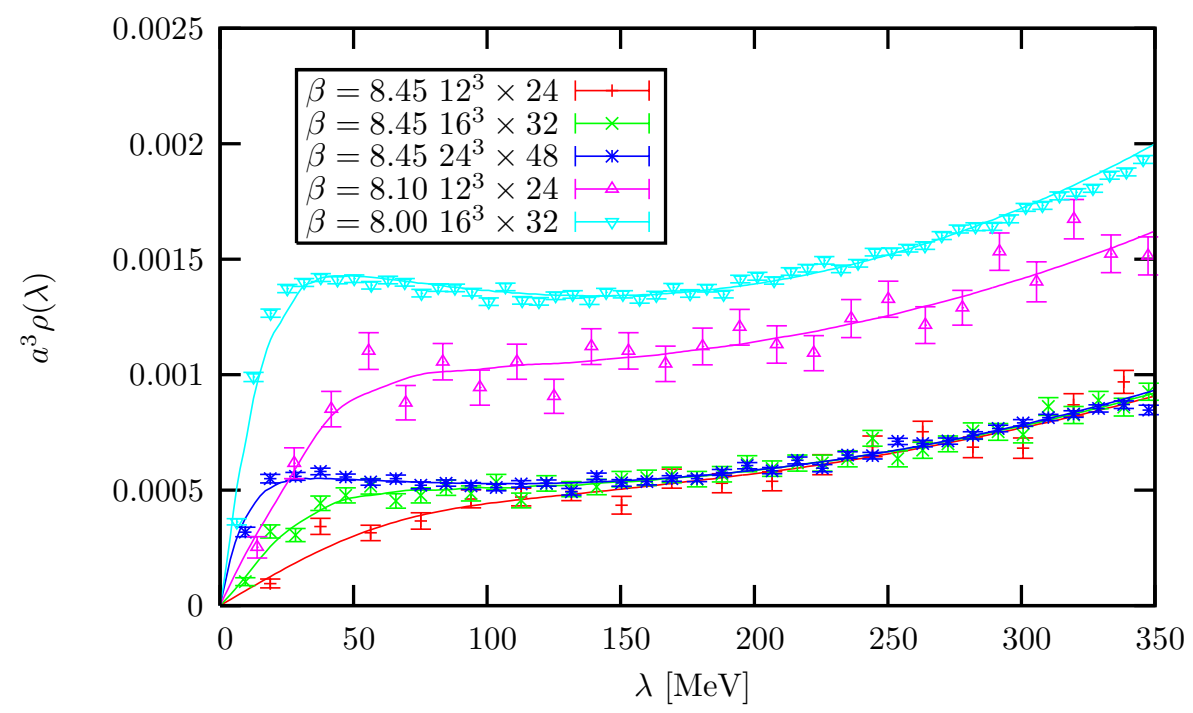

FIG. 5: The spectral densities $a^{3} \rho(\lambda, V)$ at $\beta=8.45$ for the three lattice sizes under study $\left(12^{3} \times 24,16^{3} \times 32\right.$ and $\left.24^{3} \times 48\right)$ and the spectral densities at $\beta=8.1$ and $\beta=8,0$ for lattice sizes $\left(12^{3} \times 24\right.$ and $16^{3} \times 32$, resp. $)$, together with fits using random matrix theory predictions.

$$
\rho(\lambda, V)=\Sigma_{\text {eff }} \sum_{Q} w(Q) \rho_{Q}\left(\Sigma_{\text {eff }} V \lambda\right),
$$

where $\Sigma_{\text {eff }}$ is an effective value of the chiral condensate, and

$$
\rho_{Q}(x)=\frac{x}{2}\left(J_{|Q|}^{2}(x)-J_{|Q|+1}(x) J_{|Q|-1}(x)\right)
$$

is the microscopic spectral density [76] in the sector of fixed topological charge $Q$, expressed in terms of Bessel functions $J_{n}(x)$.

We take the weights $w(Q)$ of the sectors of topological charge $Q$ (normalized to $\sum_{Q} w(Q)=1$ ) from our measured charge distributions presented in Fig. 4. To take into account the effects of higher orders in chiral perturbation theory we also add a term $a_{1} \lambda+a_{2} \lambda^{2}$ to the fitting formula (25) .

The spectral densities for the three different volumes available at the same $\beta=8.45$, and the coarser ensembles of $\beta=8.10\left(12^{3} \times 24\right)$ and $\beta=8.00\left(16^{3} \times 32\right)$, together with our fits are presented in Fig. 5.

In the region of small eigenvalues $(\lambda<100 \mathrm{MeV})$ one can see a strong volume dependence of the spectral density. This is in agreement with predictions from quenched chiral pertur- 
bation theory, where it has been shown [60] that the effective value of the chiral condensate $\Sigma_{\text {eff }}$ used in (25) diverges logarithmically as the volume is sent to infinity.

\section{Localization and fractal dimension of the eigenmodes}

In this paper, we concentrate on details of localization and fractal dimension of the overlap eigenmodes. Later on, in Section IV, similar aspects will be discussed for the topological density as derived from the overlap operator.

A useful measure to quantify the localization of eigenmodes [44, 47] is the inverse participation ratio $(\mathrm{IPR})$

$$
I(\lambda)=L_{s}^{3} L_{t} I_{2}(\lambda) \equiv L_{s}^{3} L_{t} \sum_{x} p_{\lambda}(x)^{2},
$$

with a scalar density $p_{\lambda}(x)$ that is normalized for all eigenfunctions to $\sum_{x} p_{\lambda}(x)=1$. While one would have $I=L_{s}^{3} L_{t}$ if the scalar density would have support only on one lattice point, the IPR decreases as the density becomes more delocalized, reaching $I=1$ when the scalar density is maximally spread over all lattice sites. For independent Gaussian distributed $\psi^{\sigma c}(x)$ at each site (subject to an overall normalization) the density is not maximally spread, but still delocalized. This case corresponds to $I=\pi / 2$.

The zero modes, that do not contribute to the chiral condensate, are always highly localized compared to the bulk of non-zero modes. But it turns out that the lowest non-zero modes may be localized as well. In Fig. 6, in a series of subsequent timeslices, the scalar densities of the lowest (a) and of the highest analyzed non-zero mode (b) are shown for one $Q=0$ configuration $^{1}$ of the $16^{3} \times 32$ ensemble generated at $\beta=8.45$. The lowest non-zero mode is clearly localized in a few timeslices. But the scalar density of the highest analyzed mode is also slightly inhomogeneous.

We study now more systematically the values of the IPR that occur in various parts of the spectrum. Also the zero modes are included in this discussion. In Fig. 7 histograms are shown with respect to the IPR for the complete set of all analyzed eigenmodes on the considered configurations for the five lattice ensembles, separately in bins of $\lambda$. One can see again that the zero and lower modes are remarkably more localized, whereas the bulk of the

\footnotetext{
${ }^{1}$ This configuration serves as an example for many other structural observations in this paper.
} 


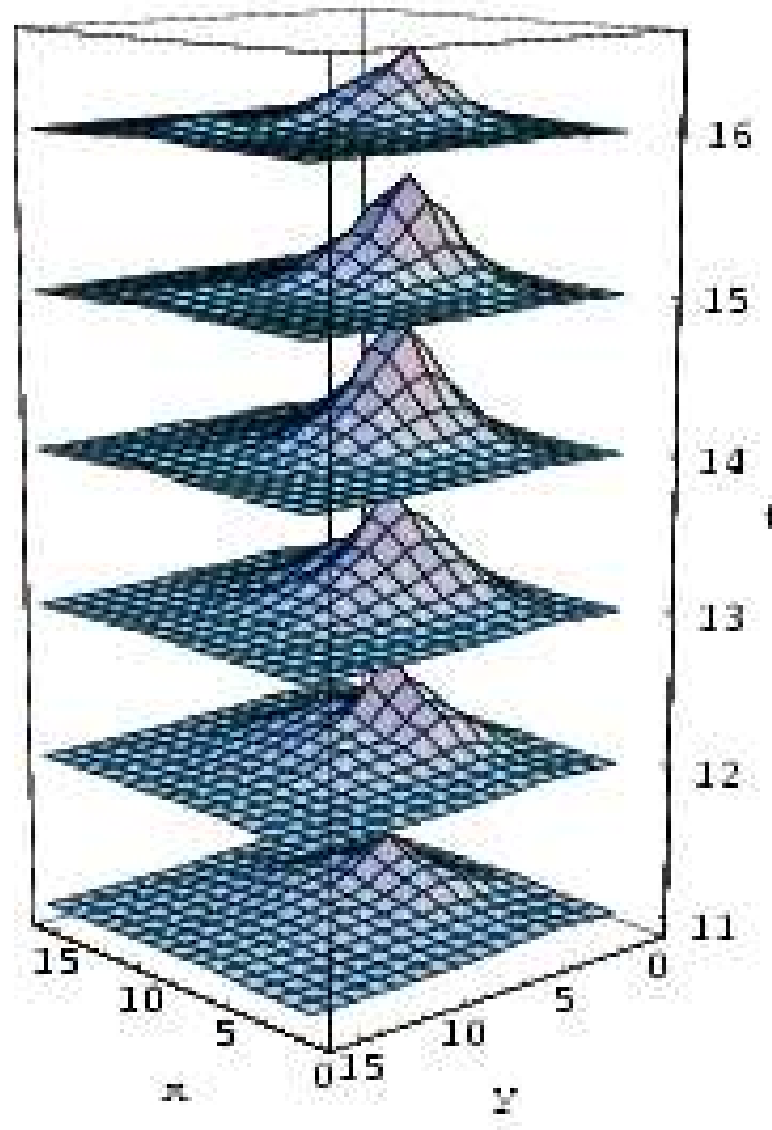

(a)

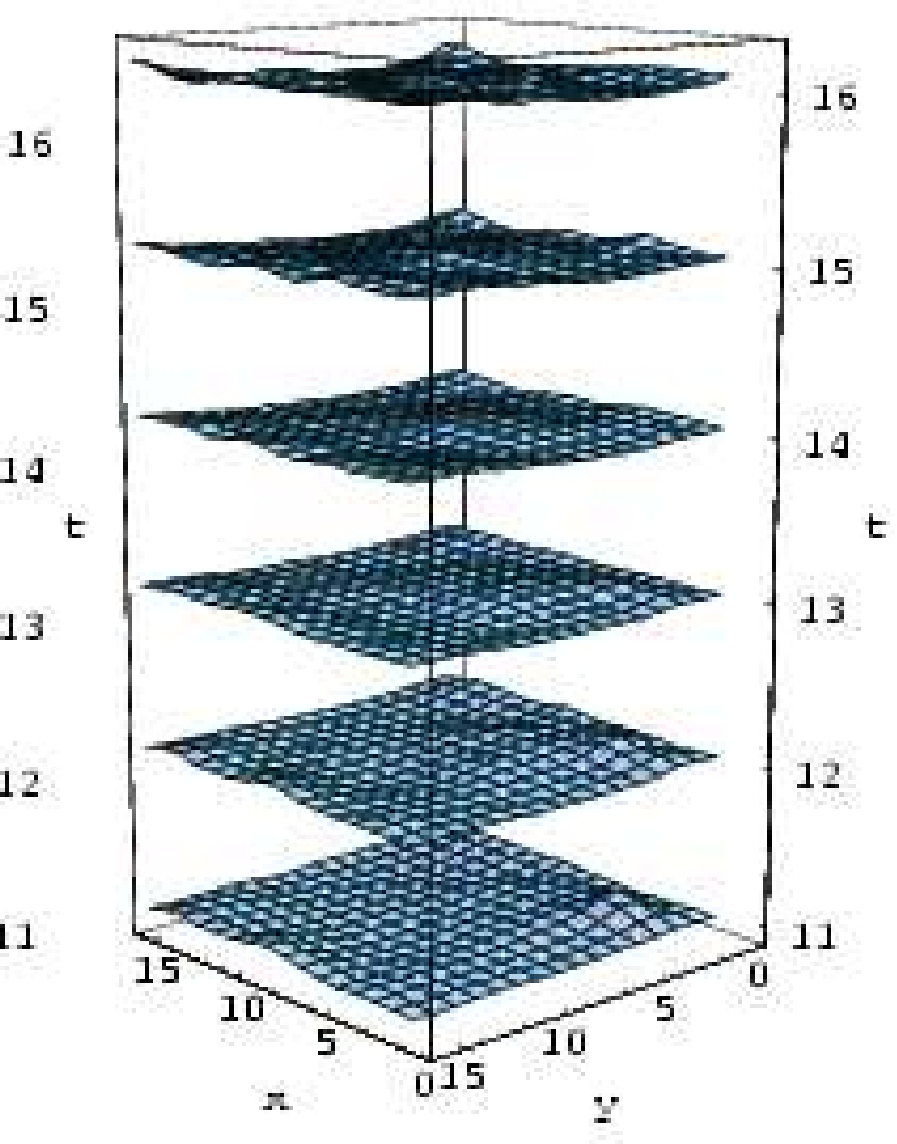

(b)

FIG. 6: The scalar density of two typical non-zero eigenmodes of the overlap operator, shown in two-dimensional profile for a series of subsequent timeslices of a $Q=0$ configuration on the $16^{3} \times 32$ lattice generated at $\beta=8.45$. (a) shows the first non-zero mode, (b) the highest (144th) analyzed mode.

higher modes is delocalized.

In Fig. 8 the IPR averaged over bins with a bin width $\Delta \lambda=50 \mathrm{MeV}$ and for the zero modes (which are considered separately) is plotted. The average IPR shows a dependence on $L_{s}$ and on the lattice spacing $a$ for zero modes and non-zero modes only in the range $\lambda<150 \mathrm{MeV}$ (for the first three bins). Beyond that interval the average IPR is practically independent of $L_{s}$ and $a$. There one finds $\langle I\rangle \leq 2.0$. It would be difficult to define an exact "mobility edge" through the localization. A proper definition would require to tell the 


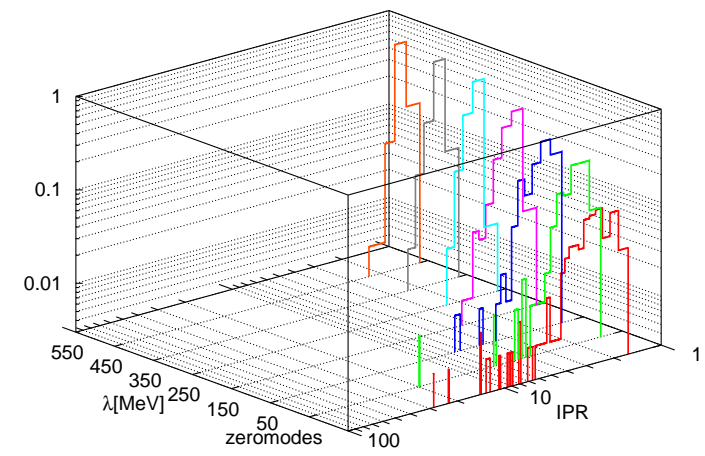

(a)

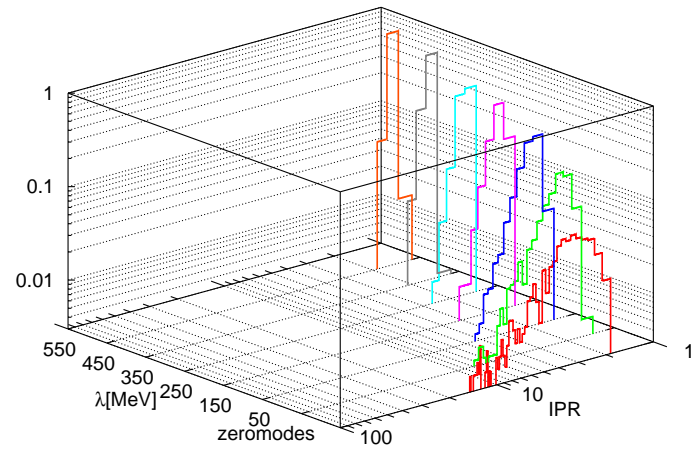

(b)

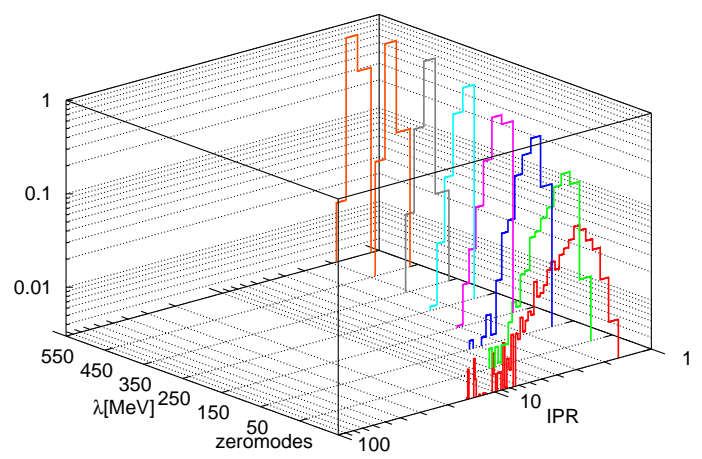

(d)

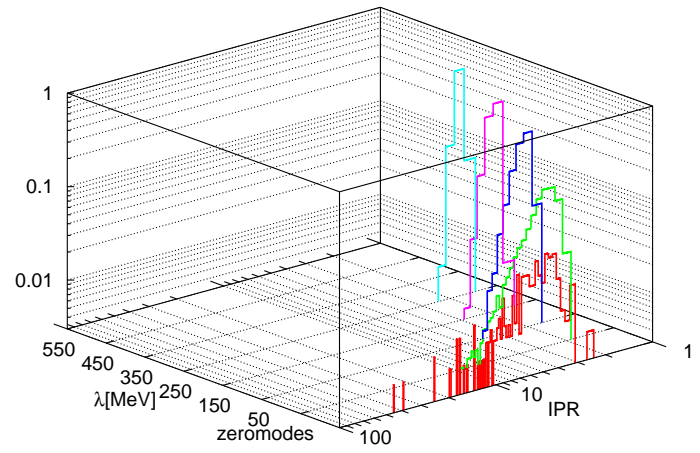

(c)

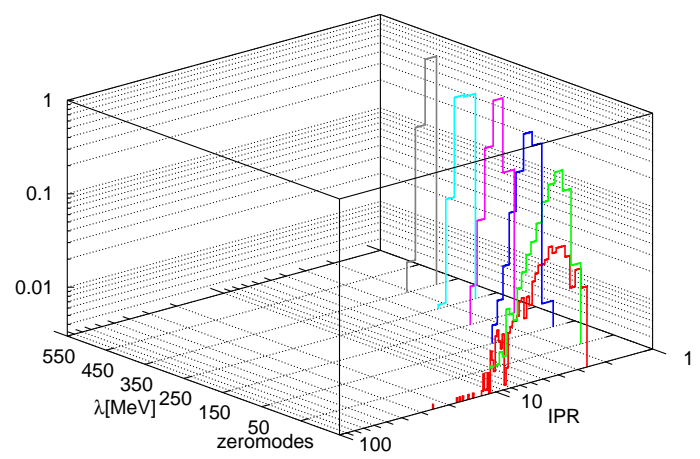

(e)

FIG. 7: Normalized histograms of IPRs as functions of $\lambda$, in subfigures ordered according to descending $\beta$ : (a) $12^{3} \times 24$ at $\beta=8.45$, (b) $16^{3} \times 32$ at $\beta=8.45$, (c) $24^{3} \times 48$ at $\beta=8.45$, (d) $12^{3} \times 24$ at $\beta=8.10$, and (e) $16^{3} \times 32$ at $\beta=8.00$, for zero modes and non-zero modes in $\lambda$ bins with width $100 \mathrm{MeV}$. 


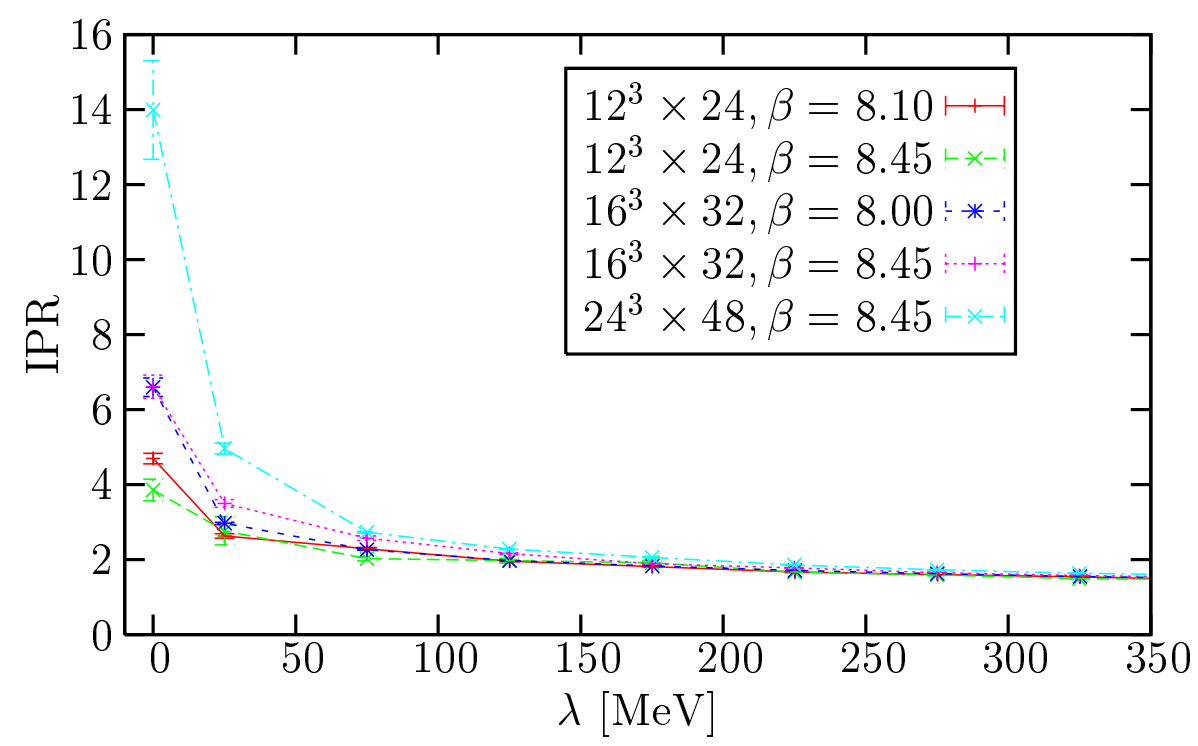

FIG. 8: The average IPR for zero modes and for non-zero modes in $\lambda$ bins of width $50 \mathrm{MeV}$ for the five ensembles.

minimal number of modes above which the pion starts propagating. ${ }^{2}$

The volume and $a$ dependence for the zero modes and the first three bins of non-zero modes allows some conclusions concerning the (fractal) dimension and the special localization properties of these modes. In view of a generalization of the IPR that will be made in a next step, we denote the effective dimension of an eigenmode based on the usual IPR as $d^{*}(2)$ (to remind us of the second moment of the scalar density $p(x)$ ). From the volume dependence of the average usual IPR, following $\langle I\rangle \propto\left(V / a^{4}\right)^{1-d^{*}(2) / 4}$ [44], one gets this dimension as an upper effective dimension. Similarly, the lattice spacing $a$ dependence can be described by $\langle I\rangle=b_{0}+b_{1} a^{d^{*}(2)-4}$. From the first bin in Fig. [ 8 it is clearly seen that the IPR grows with the physical volume $V$ at fixed $a$ and with $a^{-1}$ at fixed physical volume $V$. We fit the zero modes and the lowest non-zero modes in a couple of bins with a width $\Delta \lambda=50$ $\mathrm{MeV}$. The results of the fit are presented in the uppermost curve of Fig. 9, For the zero modes an effective dimension of $d^{*}(2)=2.2(1)$ and for the first interval of non-zero modes with $\lambda \leq 50 \mathrm{MeV}$ an effective dimension $d^{*}(2)=3.3(1)$ are found.

Gubarev et al. [46, 47] came to somewhat different conclusions guided by the $a$ dependence

\footnotetext{
${ }^{2}$ Although leading to a quantitatively wrong propagator, we will see that even the zero mode contribution alone would allow the pion to propagate.
} 
of the usual IPR $\langle I\rangle=V I_{2}$ in pure $S U(2)$ Yang-Mills theory, simulated with the Wilson action. Their fits for the $a$ dependence of the IPR, with $d^{*}(2)$ restricted to integers, gave the lowest $\chi^{2}$ per degree of freedom for the choice $d^{*}(2)=1$ for the zero modes and choosing $d^{*}(2)=0$ for the lowest non-zero modes. This discrepancy is most probably to blame to the Wilson action used to create the quenched configurations resulting in the proliferation of dislocations [52]. Our results concerning the usual IPR are similar to the findings of Aubin et al. [44].

While the usual IPR of the low modes strongly depends on $V$ and $a$, and hence the modes are localized with an effective dimension between $d^{*}(2)=2$ and 3 , the IPR of the bulk of higher modes is independent of $V$ and $a$. That means that these modes almost freely extend throughout $d^{*}(2) \lesssim d=4$ dimensions. We will see, studying the percolation behavior, that also the higher modes are not like simple plane waves, but experience a kind of soft localization. At this place it might be helpful to present in the Table II the average number of non-zero modes below some frequently used cut-off values $\lambda_{\text {cut }}{ }^{3}$.

\begin{tabular}{|c|c|c|c|c|}
\hline$\beta$ & lattice size & $a \lambda_{\text {cut }}$ & $\lambda_{\text {cut }}$ & average \# of NZM \\
\hline \hline 8.45 & $16^{3} \times 32$ & 0.1064 & $200 \mathrm{MeV}$ & $13.1(2)$ \\
& & 0.2128 & $400 \mathrm{MeV}$ & $36.2(2)$ \\
& & 0.3374 & $634 \mathrm{MeV}$ & $92.3(2)$ \\
\hline 8.10 & $12^{3} \times 24$ & 0.14 & $200 \mathrm{MeV}$ & $11.0(2)$ \\
& & 0.28 & $400 \mathrm{MeV}$ & $28.1(2)$ \\
& & 0.42 & $600 \mathrm{MeV}$ & $59.3(2)$ \\
& & 0.56 & $800 \mathrm{MeV}$ & $117.3(4)$ \\
\hline
\end{tabular}

TABLE II: The average number of non-zero modes (NZM) below various spectral cut-offs for two ensembles used in this study. The cut-offs are given both in $\mathrm{MeV}$ and in $1 / a$.

In the theory of the metal-insulator transition tools for a quantitative description of

\footnotetext{
${ }^{3}$ In the following the cut-off values $200,400,600$ and $800 \mathrm{MeV}$ on the $12^{3} \times 24$ lattice at $\beta=8.1$ should be considered as approximate values. Using the interpolated value for the lattice spacing at $\beta=8.1$, the exact values are 195, 389, 584 and $778 \mathrm{MeV}$, respectively.
} 
critical level statistics are given and the multifractal properties of the eigenfunctions are in the focus of interest. For this purpose, the notion of IPR has been generalized [77, 78] to

$$
I_{p}(\lambda)=\frac{\sum_{x, n}\left|\psi_{\lambda_{n}}(x)\right|^{2 p} \Theta_{\epsilon}\left(\lambda-\lambda_{n}\right)}{\sum_{n} \Theta_{\epsilon}\left(\lambda-\lambda_{n}\right)},
$$

with a free parameter $p$ and a window function $\Theta_{\epsilon}\left(\lambda-\lambda_{n}\right)=1 / \epsilon$ for $\left|\lambda-\lambda_{n}\right| \leq \epsilon / 2$ and vanishing elsewhere. Depending on whether the metal phase, the insulator phase or the critical region is met, this quantity would scale in a different way with the volume of the specimen (see Table III, results see Fig. 9)).

\begin{tabular}{|l|l|l|}
\hline$I_{p}(\lambda) \propto L^{-d(p-1)}$ & metallic & electrons propagate freely \\
\hline$I_{p}(\lambda) \propto L^{-d^{*}(p)(p-1)}$ & critical & $\begin{array}{l}\text { electrons propagate along } \\
\text { low-dimensional structures }\end{array}$ \\
\hline$I_{p}(\lambda)=\mathrm{const}$ & insulator & electrons do not propagate \\
\hline
\end{tabular}

TABLE III: Volume scaling of generalized IPR's according to the metal-insulator transition analogy.

For the "metallic" phase, $d$ is the embedding dimension of the system, in our case $d=$ 4. For the critical region the effective dimension becomes $d^{*}(p)<d$. In this sense, all eigenstates with $\lambda<200 \mathrm{MeV}$ definitely belong to a "critical region". From the standard IPR $I=L_{s}^{3} L_{t} I_{2}(p=2)$ we have concluded that the dimension of the zero modes is close to two. In the next bins of $50 \mathrm{MeV}$ each, the dimension quickly exceeds three, before it is finally approaching four.

For the "critical region" and up to $\lambda=400 \mathrm{MeV}$ we present now the results for the higher order $p$ fractal dimensions $d^{*}(p)$, which allows to recognize a varying type of localization at various levels (heights) of the scalar density $p(x)$. Notice the decrease of the multifractal dimension with increasing $p$, resulting from the fact that $I_{p}$ explores regions of higher scalar density. For $p=4$, for example, one sees generally a reduced dimension compared to $p=2$, interpolating between "filamentary" $\left(d^{*}(4) \approx 1\right.$ for zero modes $)$ and "surface-like" $\left(d^{*}(4) \approx 2\right.$ for the next two bins with $\lambda<100 \mathrm{MeV}$ ), becoming $d^{*}(4) \approx 3.5$ at higher $\lambda$. This indicates that the regions of higher scalar density are geometrically distinct from those regions where an eigenmode is present only with a tail of the scalar density. For $p=20$ - 30 the analyzed 


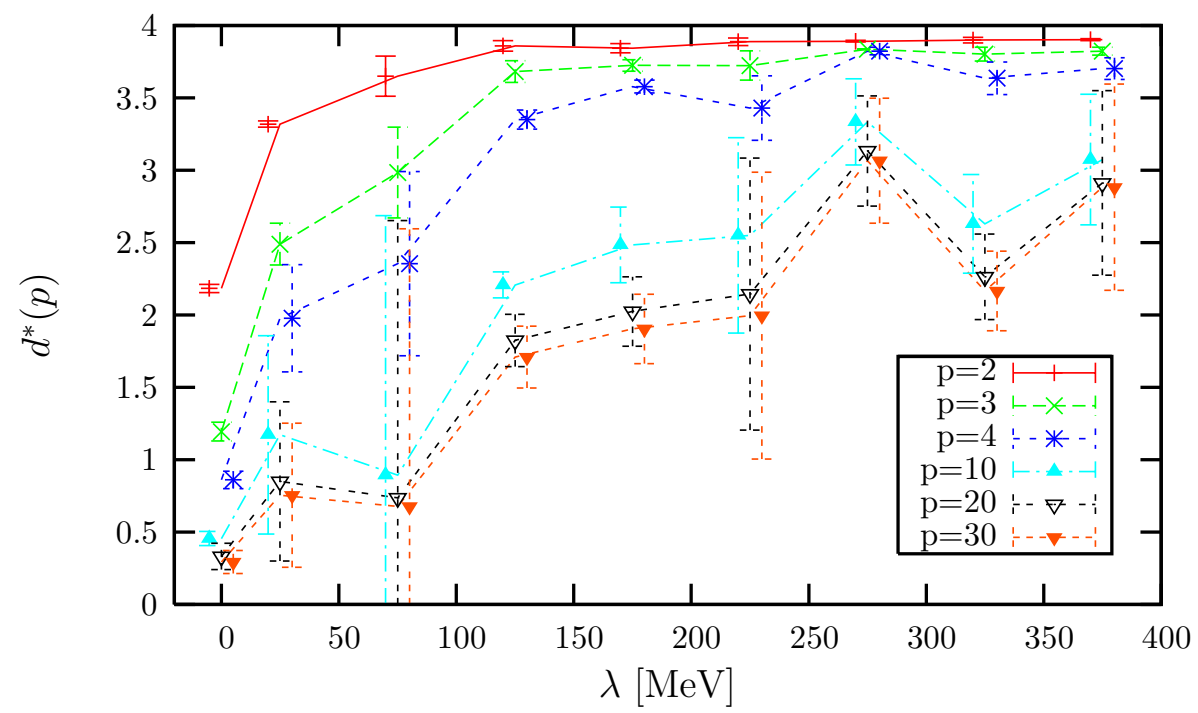

FIG. 9: The fractal dimension $d^{*}(p)$ obtained from fits of the volume dependence of the averages of the generalized $I_{p}$, presented for zero modes and for non-zero modes in $\lambda$ bins of width $50 \mathrm{MeV}$ for the three ensembles with different volume and common $\beta=8.45$.

fractal dimension does not grow anymore. The envelope tells that, with respect to the maxima of the scalar density, zero modes and non-zero modes up to $\lambda=100 \mathrm{MeV}$ are all characterized by a less than one-dimensional localization (i.e. isolated peaks), whereas in the following spectral region two-dimensional or three-dimensional localization prevails for the regions of highest scalar density (cf. Fig. 6). In contrast to this, the standard IPR based on $I_{2}$ alone cannot resolve these details and would describe the modes in the spectral region $\lambda>100 \mathrm{MeV}$ as four-dimensional.

It is tempting to conjecture a pinning-down of the low-dimensional low-lying modes to specific confining objects (vortices, monopoles, close-by meron pairs etc.) and a relation to the localization of topological charge (see Section IV in this context). It is important to recognize that the difference between the zero modes and the lowest (say, 10) non-zero modes is less pronounced than it seems in the result of binning. In marked contrast to this, the highest analyzed non-zero modes are really qualitatively different. This could already be concluded from Fig. 6.

We show in Fig. 10 (a) the result of a cluster analysis ${ }^{4}$ of 170 configurations on the $16^{3} \times 32$

\footnotetext{
4 The cluster analysis with respect to some observable was introduced in Section IE
} 


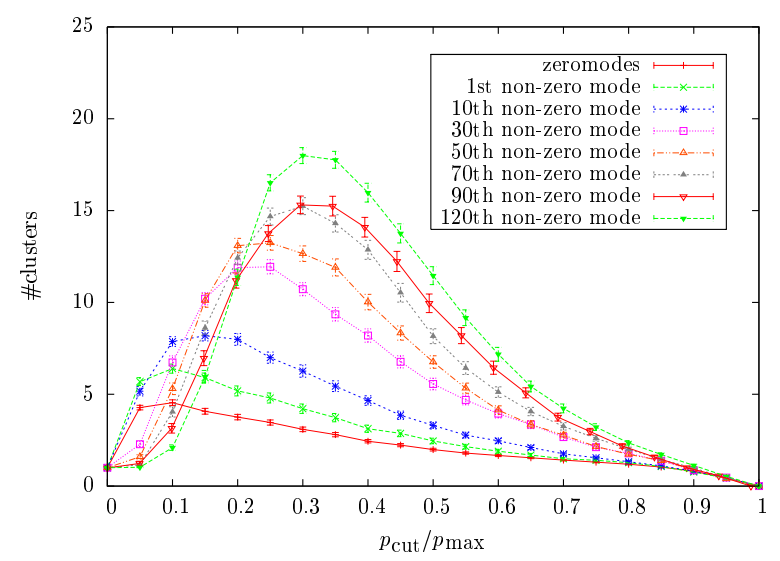

(a)

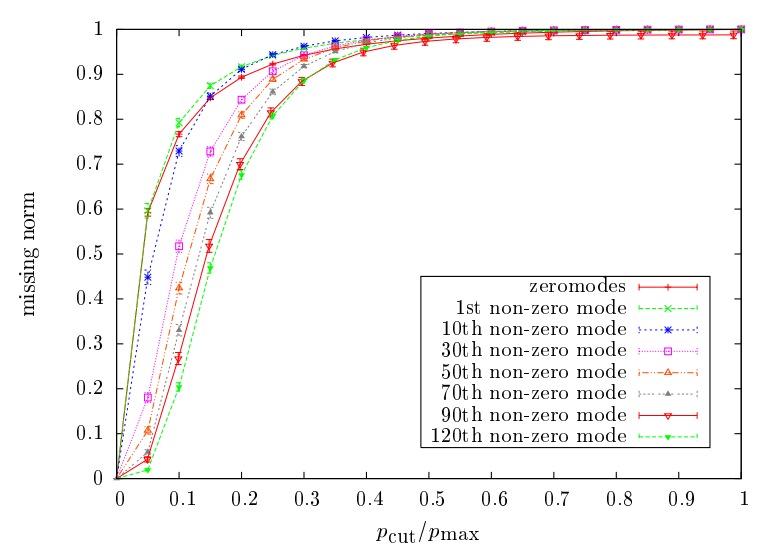

(c)

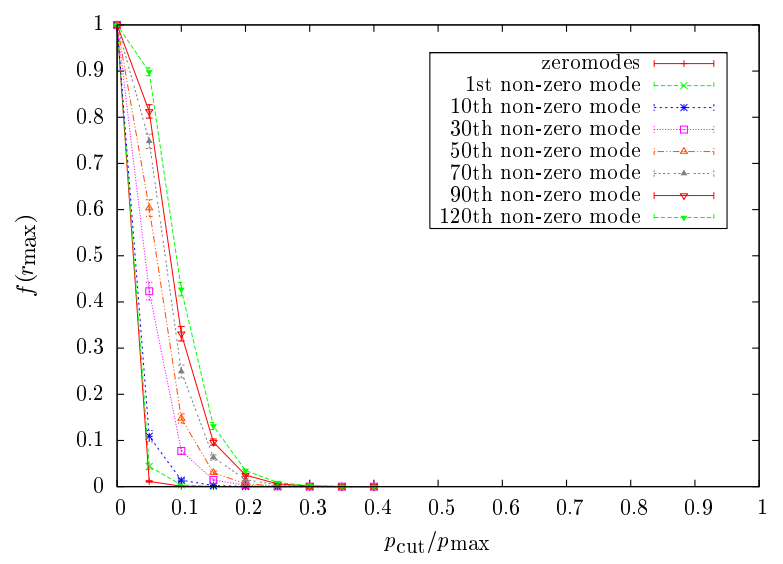

(b)

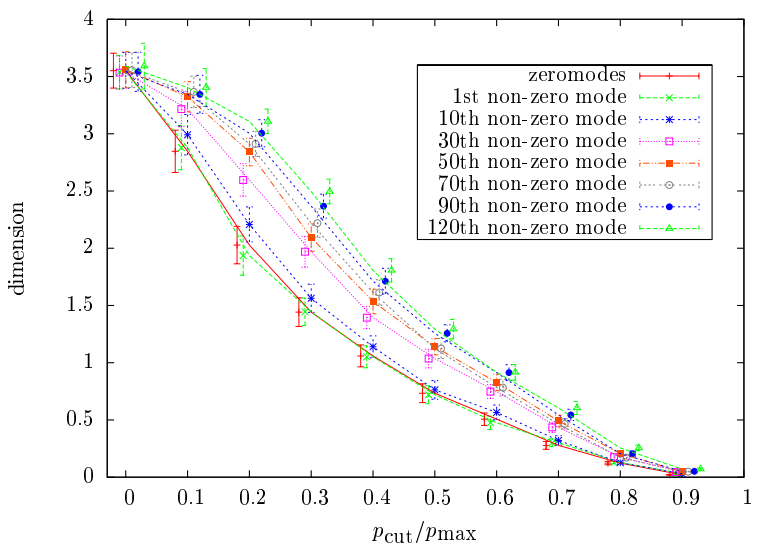

(d)

FIG. 10: Cluster analysis of some individual eigenmodes as listed in the legend box, averaged over 170 configurations on $16^{3} \times 32$ generated at $\beta=8.45$. The $p_{\text {cut }} / p_{\max }$ dependence is shown of (a) the total number of separate clusters at the height $p_{\text {cut }}$, (b) the connectivity as a signal for the percolation of the mode, (c) the missing norm of the mode and (d) the effective dimension of the mode at height $p_{\text {cut }}$ determined by the return probability (22) of random walkers.

lattice generated at $\beta=8.45$. The average is over the zero modes in this subensemble, the 1 st, the 10 th, the 30 th etc. up to the (arbitrarily chosen) 120th non-zero mode. We analyze the average cluster composition as function of the cut-off scalar density $p_{\text {cut }} / p_{\text {max }}$ (relative to the maximum of the scalar density of the respective $\left.\operatorname{mode} p_{\max }=\max _{x} p_{\lambda}(x)\right)$. In this type of cluster analysis one attempts to decompose the set of lattice points with $p_{\lambda}(x) \geq p_{\text {cut }}$ into one or more connected clusters, each enclosing secondary maxima of $p_{\lambda}(x)$. For the highest analyzed mode, beginning at $p_{\text {cut }}=0.9 p_{\max }$, rapidly further maxima become visible, and 
the number of separate clusters grows to $\approx 20$ at $p_{\text {cut }}=0.35 p_{\text {max }}$. In contrast to this, the typical zero mode only slowly develops up to $\approx 5$ maxima at $p_{\text {cut }}=0.1 p_{\max }$. From the zero mode to the highest analyzed mode there seem to exist several centers that "attract" the mode, but the mechanism might be different. The lowest modes might be pinned down to some lumps of topological charge (á la Diakonov and Petrov [79], whereas the localization of the highest (analyzed) modes might be the result of Anderson-like localization in the random gauge field background.

The lowest ten non-zero modes are very similar to the typical zero mode. The zero modes percolate, i.e. extend over the full lattice, only at a height below $p_{\text {cut }}=0.25 p_{\text {max }}$.

The highest analyzed non-zero mode percolates at $p_{\text {cut }}=0.4 p_{\max }$ (with the maximal scalar density being smaller, of course). This can be seen in Fig. 10 (b). The difference in the percolation behavior can be concluded also from the missing norm [80]

$$
R\left(p_{\text {cut }}\right)=1-\sum_{\left\{x \mid p(x) \geq p_{\text {cut }}\right\}} p(x)
$$

as function of $p_{\text {cut }} / p_{\max }$ that is shown in Fig. 10 (c). Here the similarity between the zero modes and the first 10 non-zero modes is similarly clear.

At each height level of $p_{\lambda}$ the effective shape of the leading cluster of the respective mode can be explored by the random walk method described in section IIF. In the present context one studies how the return probability of the walkers to the maximum of $p_{\lambda}(x)$ decreases with an increasing number $\tau$ of steps. A fit of the power law decrease (22) provides the effective dimensions $d^{*}$ shown in Fig. 10(d). We notice that the average dimension averaged over the 10 lowest modes continuously rises from $d^{*}=0$ to $d^{*}=3.5$. It becomes threedimensional at the same $p_{\text {cut }} / p_{\text {max }}$ when the missing norm begins the final steep drop from $80 \%$ to $0 \%$. As for the 120 th non-zero mode, the effective dimension $d^{*}$ rises more steeply and reaches $d^{*} \approx 3$ at higher $p_{\text {cut }} / p_{\max }$ when the missing norm is already dropped to 60 $\%$. For a survey of dimensionalities of overlap eigenmodes see Tables VII and VIII in the Appendix. 


\section{Chiral properties of the non-zero modes}

In this section we characterize the different parts of the low-lying spectrum of non-zero modes by the distribution of sites over the local chirality. Later on, the local chirality of the lowest modes is shown to be locally correlated to the topological density.

In a given lattice point $x$, one might be interested in the intensity ratio

$$
r_{\lambda}(x)=\frac{p_{\lambda+}(x)}{p_{\lambda-}(x)}
$$

of the two chiral projections for a given mode $\psi_{\lambda}$. For the chiral zero modes this ratio is $\infty$ or 0 everywhere. For the non-chiral, non-zero modes it is some function of $x$. It is useful to relate $r_{\lambda}(x)$ to $[50]$

$$
X_{\lambda}(x)=\frac{4}{\pi} \arctan \left(\sqrt{r_{\lambda}(x)}\right)-1 \in[-1,+1] .
$$

It will be seen that relatively low-lying modes have regions where the local chirality carries information about the (anti-)selfdual character of the background field, to be precise, where $0.5<|X(x)|<1$.

In Fig. 11 and Fig. 12 normalized histograms with respect to the local chirality $X(x)$ are shown for the $Q=0$ subsample (containing 37 configurations) of the $16^{3} \times 32$ lattices generated at $\beta=8.45$ with the lattice sites selected according to the scalar density $p(x)$. The left figures show the 10 different $X$-histograms for the lowest 20 individual non-zero modes (the pair with $\lambda$ and $-\lambda$ gives rise to the same histogram), whereas the right figures show the histograms where all non-zero modes belonging to a $\lambda$ bin (with a bin size $100 \mathrm{MeV}$ ) are contributing to 8 histograms covering the spectral range up to $800 \mathrm{MeV}$. The different rows show different cuts applied restricting the lattice sites $x$ to those with $p_{\lambda}(x) \geq p_{\text {cut }}$. The cuts are chosen such to represent $1 \%, 6.25 \%, 12.5 \%$ and $50 \%$ of all lattice sites.

For zero modes (not included here) the histograms would be $\delta$-functions at $X=-1$ or $X=1$ independent of any cut with respect to the scalar density. Although being non-chiral when integrated over space-time, the first non-zero modes locally may have a considerable degree of chirality (peaking in $X$ somewhere between 0.75 and 0.9 ) for the leading $1 \%$ or

$6 \%$ of the lattice sites with the highest scalar density. It turns out that with a higher, more restrictive cut the degree of local chirality can be enhanced. A similarly stronger effect can be seen towards the lower modes. That means that the 5 - 10 maxima of the scalar density 


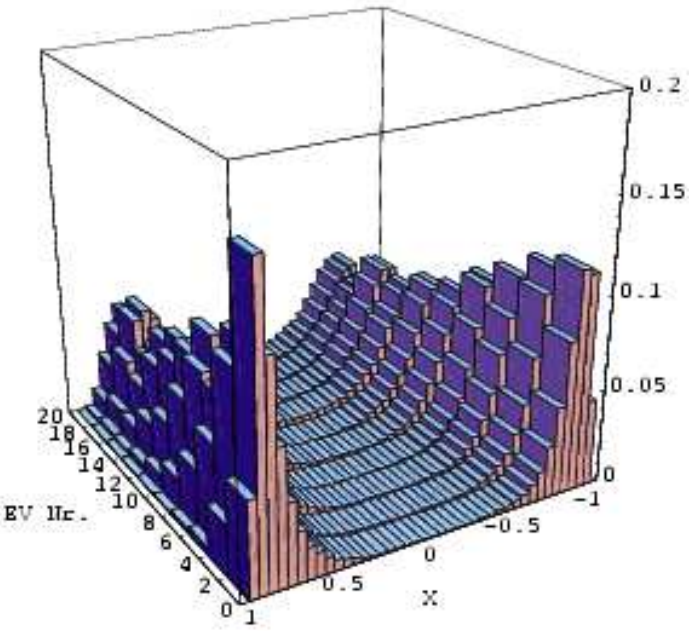

(a)

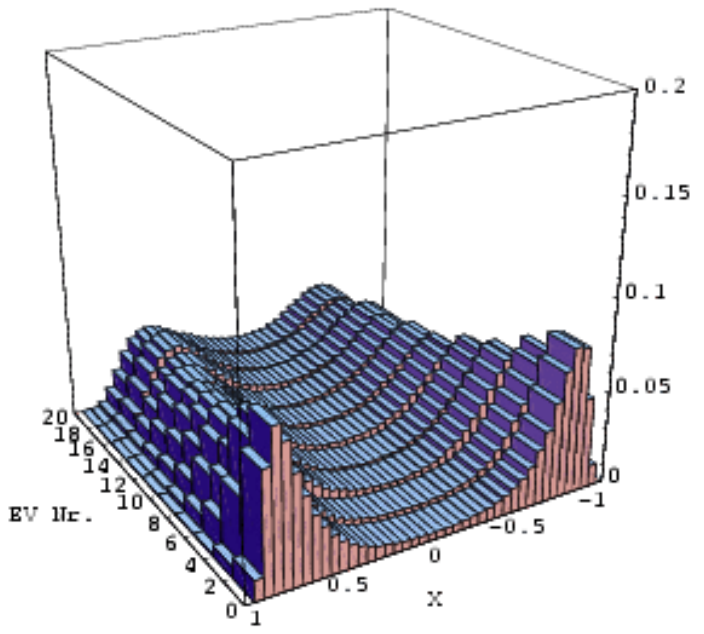

(c)

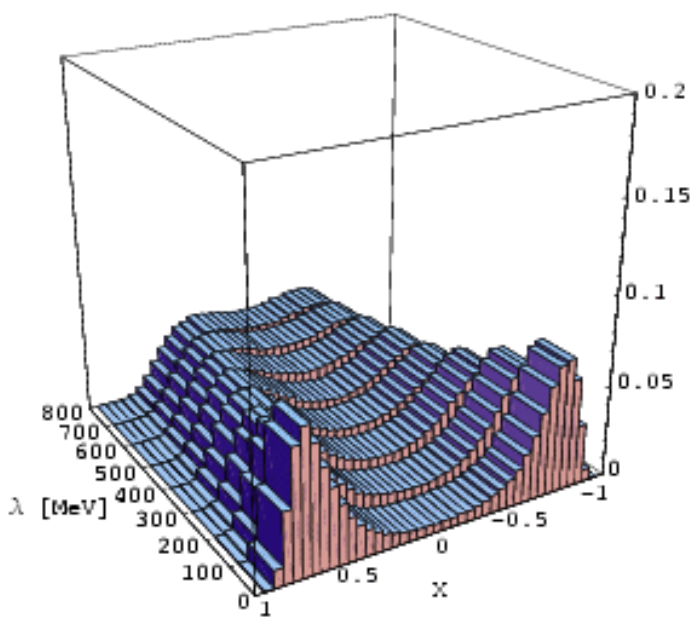

(b)

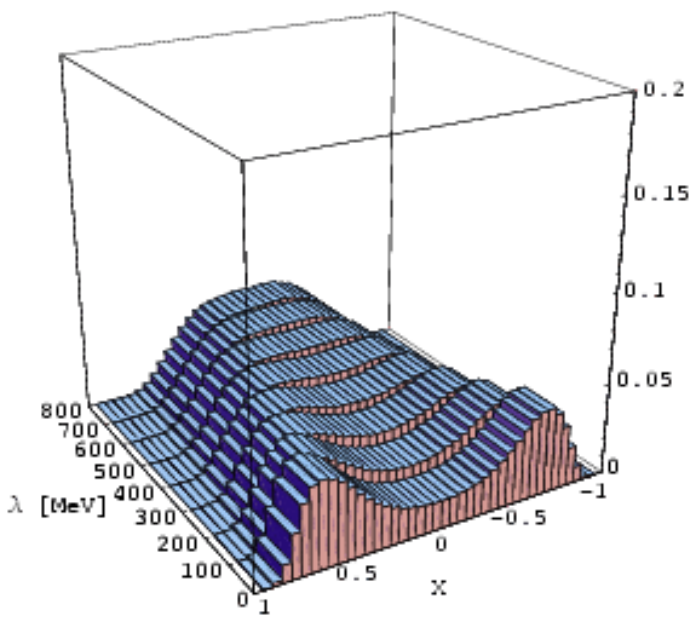

(d)

FIG. 11: Normalized histograms of the local chirality in the $Q=0$ subsample (consisting of 37 configurations) of the $16^{3} \times 32$ lattices generated at $\beta=8.45$. Left: for the lowest ten pairs (with positive and negative $\lambda$ ) averaged over the subsample. Right: for all modes from the subsample, averaged over $\lambda$ bins of width $100 \mathrm{MeV}$. Upper row: with a cut for $1 \%$ of the sites with biggest scalar density $p(x)$, lower row: with a cut for $6.25 \%$ of the sites with biggest $p(x)$. 


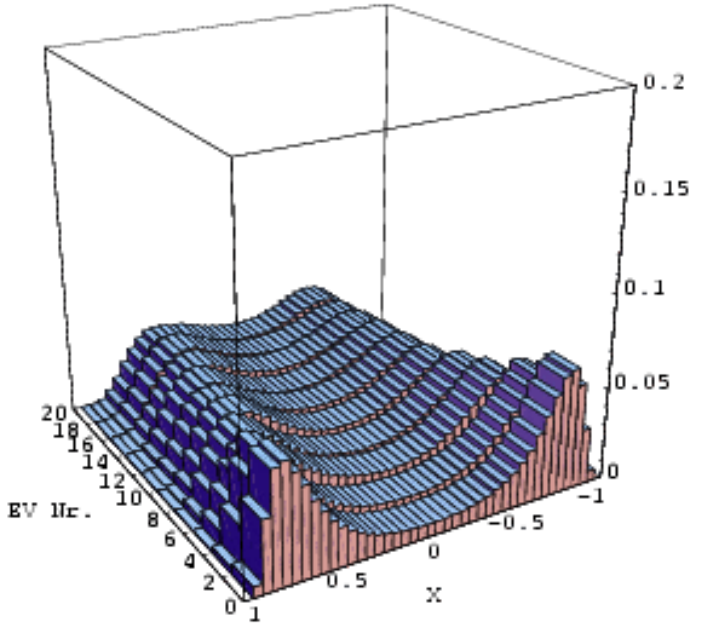

(a)

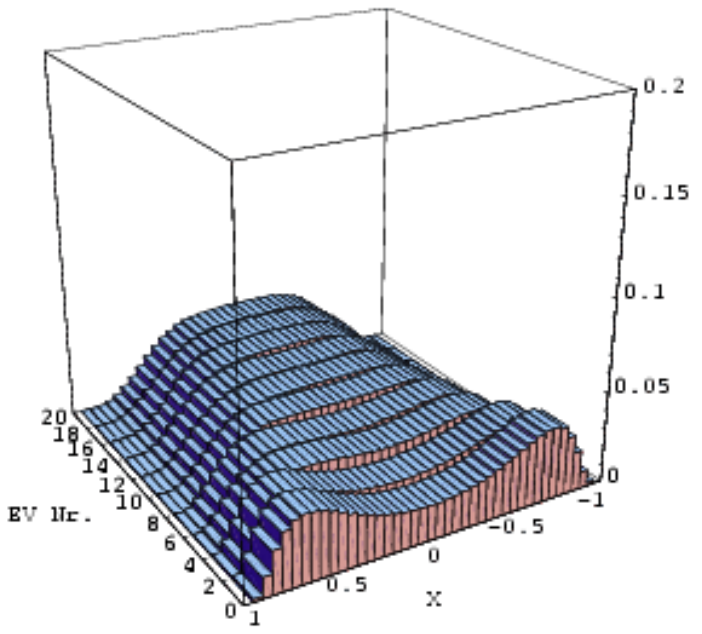

(c)

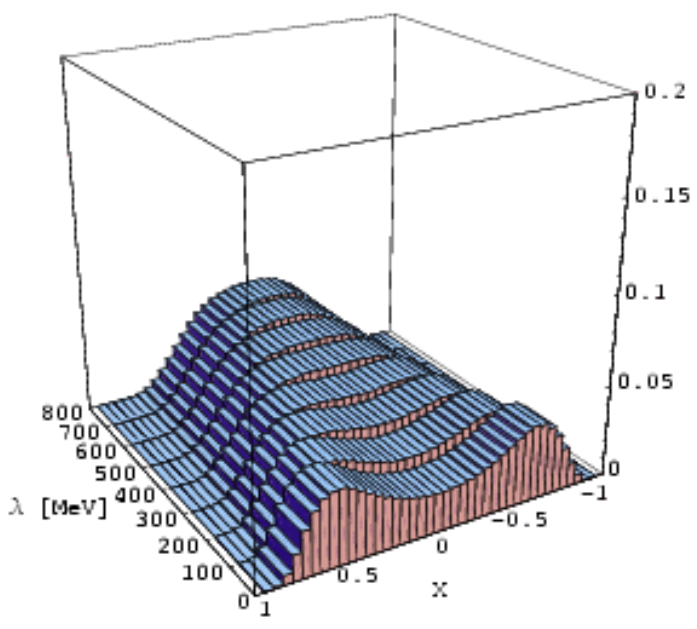

(b)

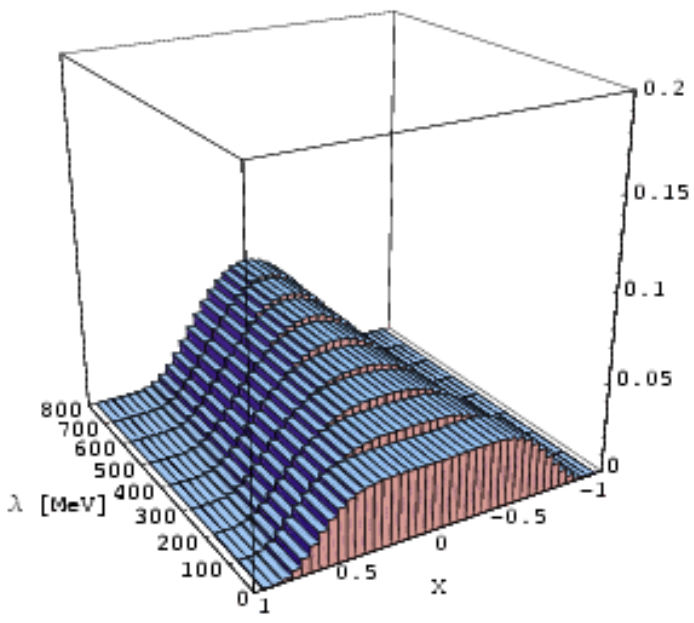

(d)

FIG. 12: The same as Fig. 11 but with less exclusive cuts with respect to the scalar density: Upper row: with a cut for 12.5 /lower row: with a cut for $50 \%$ of the sites with biggest $p(x)$. 
of the lowest non-zero modes are localized in space-time region where the local chirality is strongly correlated to the topological charge density of the gluonic background. The highest modes, as we have found, have $O(20)$ local maxima. In contrast to the lower modes, the histogram of local chirality for the higher ones is dominated by a broad bump of low chirality $X(x)$ distributed around zero for all cuts in $p(x)$. This means that the maxima (localization) of the highest modes are not the result of pinning down at spots of topological charge density, rather they are the result of waves scattering on a random gauge field background.

One can see that the eigenmodes with eigenvalues up to $200 \mathrm{MeV}$ show an enhancement of local chirality around $X= \pm(0.5 \ldots 0.75)$ as long as one focusses on the $6 \%$ of the lattice points with the strongest scalar density. If $50 \%$ of the lattice sites are included in the analysis, only the first two pairs of non-zero eigenmodes still show some enhancement of local chirality around $X= \pm 0.75$.

We will later (at the end of Section IVE) consider the correlation function between the topological charge in the gluonic background and the local chirality $X(x)$ of individual non-zero modes. The result establishes that they are interrelated over some distance in space-time.

\section{TOPOLOGICAL CHARGE DENSITY FROM THE OVERLAP OPERATOR}

\section{A. Two-point function of the all-scale and the mode-truncated density}

The topological charge density of the gluonic field seems to play a deciding role for the localization of the lowest modes. In this Section we are studying the properties of the overlap definition of the topological density according to Eqs. (18) and (19). The striking difference between the all-scale topological charge density and the mode-truncated one can be illuminated studying the point-to-point correlator of the topological density. Formally, the definition of the two-point function is the same for $q(x)$ and $q_{\lambda_{\text {cut }}}(x)$ :

$$
C_{q q}(r)=\frac{\sum_{x, y}\langle q(x) q(y)\rangle \delta(r-|x-y|)}{\sum_{x, y} \delta(r-|x-y|)} \leq 0 \quad \text { for } \quad r>0 .
$$

Reflection positivity, $i$. e. the pseudoscalar nature of $q(x)$ together with positivity of the metric in Hilbert space, demands that the topological charge density correlator is negative for 


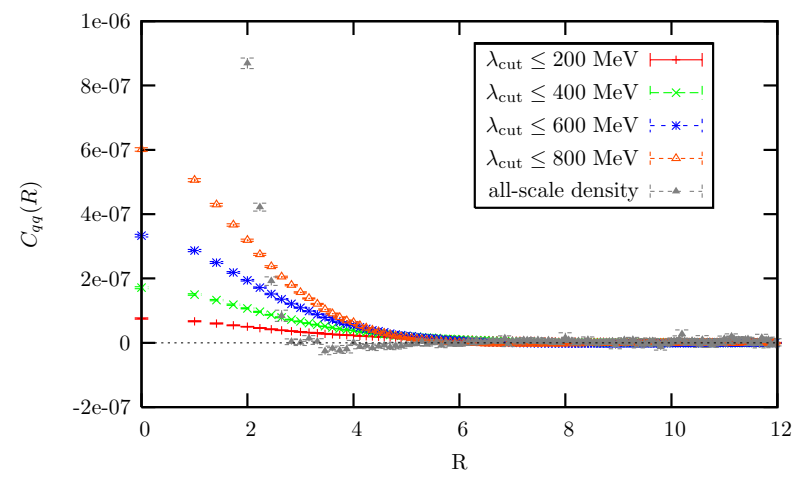

(a)

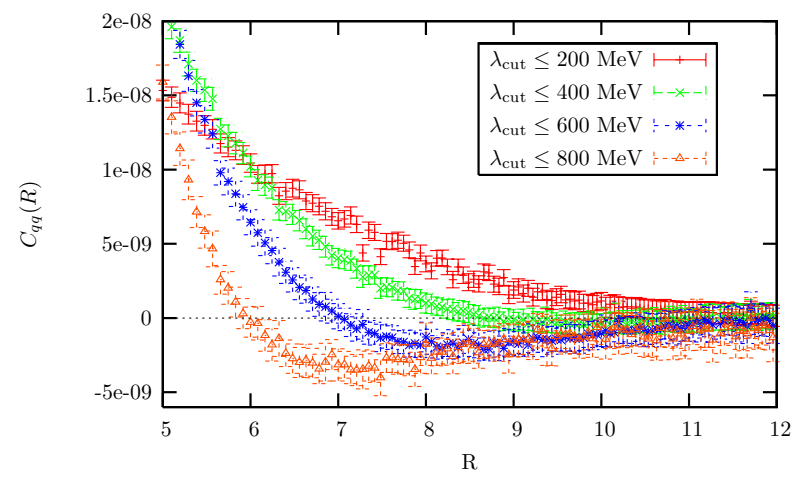

(b)

FIG. 13: The topological charge density correlator for the $12^{3} \times 24$ lattice configurations at $\beta=8.10$. (a) The correlator of the mode-truncated density $q_{\lambda_{\text {cut }}}(x)$ for various $\lambda_{\text {cut }}$ compared with the correlator of the all-scale density $q(x)$, measured only on 53 configurations. The right figure (b) magnifies the region in $r$ where the mode-truncated correlators become negative for sufficiently large $\lambda_{\text {cut }}$.

all distances, $C_{q q}(r) \leq 0$ for $\mathrm{r}>0$. Of course, the vacuum expectation value $\langle q(x) q(y)\rangle$ must be a function only of the distance $r=|x-y| \geq 0$, due to Euclidean rotational invariance.

While the topological charge correlator $C_{q q}(r)$ in the continuum should be negative for nonvanishing distance, the topological susceptibility, which is the integral over the correlator $\chi_{t}=\int d x\langle q(0) q(x)\rangle$, must be positive. To solve the apparent contradiction, formally divergent contact counterterms of the form $C_{q q}(x) \rightarrow C_{q q}(x)+c_{1} \delta(x)+c_{2} \Delta \delta(x)+c_{3} \Delta^{2} \delta(x)$ have to be introduced in the continuum theory [81].

Since overlap fermions are not ultralocal, the fermion action cannot be strictly reflection positive. Because the overlap operator $D(0)$ has a finite range in space-time (see Fig. 2) practically independent of $\beta$, we expect that the correlator $C_{q q}(r)$ will have a positive core with a fixed width in lattice units. The negative tail beyond the core radius corresponds to the above mentioned theoretical requirement. This has been discussed recently in Ref. [61].

For the correlator of the mode-truncated density $q_{\lambda_{\text {cut }}}(x)$ one would expect that this structure is smoothed out and that below some $\lambda_{\text {cut }}$ the corresponding two-point function will be positive at all distances. In Fig. 13 we show the correlator of the mode-truncated 


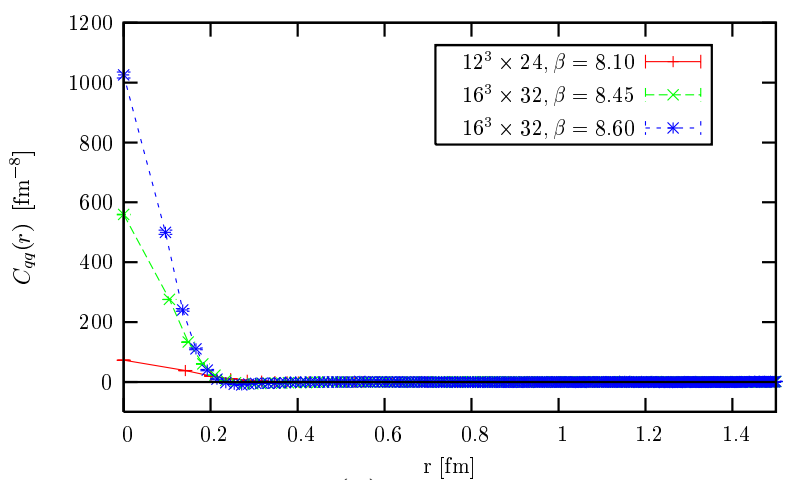

(a)

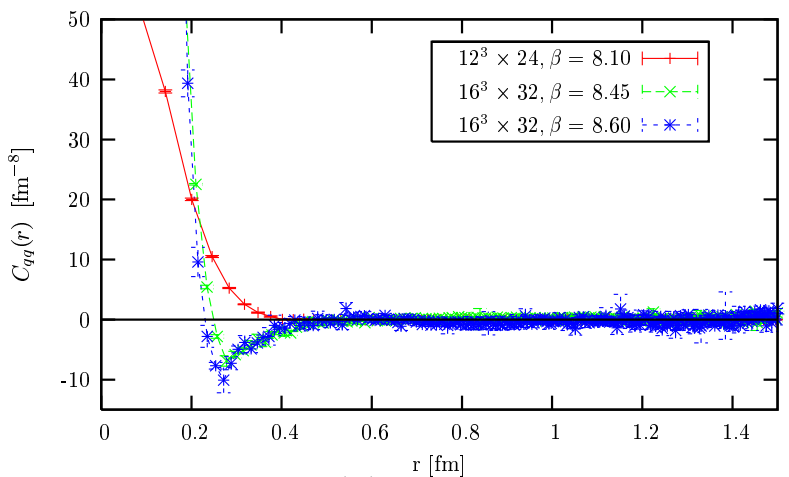

(b)

FIG. 14: Comparison of the topological charge density correlator based on the all-scale density $q(x)$ for the $12^{3} \times 24$ lattice at $\beta=8.10$ (53 configurations) with the same correlator for the $16^{3} \times 32$ lattice at $\beta=8.45$ (only 5 configurations) and $\beta=8.60$ (2 configurations). The right figure (b) magnifies the region in $r$ where the correlator becomes stronger negative when one gets closer to the continuum limit.

topological charge density for various $\lambda_{\text {cut }}$ for the $12^{3} \times 24$ lattice configurations generated at $\beta=8.10$. In Fig. 13 (a) they are compared with the correlation function of the all-scale topological density measured on 53 configurations. In Fig. 13 (b) we show the correlator only for different mode-truncated densities, however magnified, in the region of $r$ where they start to develop a negative tail for sufficiently high cut-off. This happens at $R \geq 6 a$ for $\lambda_{\text {cut }}=800 \mathrm{MeV}$, at $R \geq 7 a$ for $\lambda_{\text {cut }}=600 \mathrm{MeV}$, and at $R \geq 9 a$ for $\lambda_{\text {cut }}=400 \mathrm{MeV}$. As long as it were positive, this correlator would be interpreted in terms of the number density and the convoluted profile of effective topological charge clusters. It is interesting, that higher resolution in the present case (similarly to doing less smearing [82] or doing restricted cooling [24, 83]) results in effectively attractive correlations between opposite sign topological charge clusters not only in full QCD but also in quenched gauge fields as emphasized in Ref. [82]. Since the total topological charge does not depend on $\lambda_{\text {cut }}$, the growing negative tail must be compensated in the susceptibility $\sum_{x} C_{q q}(|x|)$ by a growing positive core with increasing $\lambda_{\text {cut }}$.

In order to demonstrate how the correlation function of the all-scale topological charge density varies with the lattice spacing we compare in Fig. 14 the correlator for $12^{3} \times 24$ lattices at $\beta=8.10$ (53 configurations) with the correlator measured on $16^{3} \times 32$ lattices at $\beta=8.45$ (only 5 configurations) [6] and $16^{3} \times 32$ lattices at $\beta=8.6$ (2 configurations). 
An extrapolation of the function $a(\beta)$ to the highest $\beta$ gives $a(\beta=8.6)=0.096(1)$. The volumes of the first two lattices are approximately equal. The required negativity of the correlator develops only for a sufficiently fine lattice. The much bigger number of clusters at higher values $|q(x)| \approx 0.25 q_{\max }$ (see Section IVC) seems to be necessary to achieve this. Thus, the cluster multiplicity of the all-scale topological density simply reflects the size of the lattice in lattice units.

\section{B. Two-dimensional profile seen with varying resolution}

As a vacuum expectation value, the point-to-point correlation function obviously has to be rotational invariant. Individual lattice configurations, however, are richer in structure and necessarily have a locally anisotropic structure. This results from lower-dimensional structures present in the configurations that locally break rotational invariance.

As an example, a snapshot with four levels of resolution is presented in Fig. 15 of the same two-dimensional section through the typical configuration, already considered in Figs. [6 and 24, of the ensemble of $16^{3} \times 32$ lattices at $\beta=8.45$. The respective resolution can be inferred from the corresponding $\lambda_{\text {cut }}$. The two-dimensional section is periodically doubled in the two directions such that the one-dimensional structure (apparently one "ridge" per elementary cell in this two-dimensional world) can be recognized. In particular, this ridge is clearly visible at $\lambda_{\text {cut }}=200$ and $400 \mathrm{MeV}$, being already less clearly visible at $\lambda_{\text {cut }}=634 \mathrm{MeV}$. At a resolution of $400 \mathrm{MeV}$ and higher, also single peaks (a 0-dimensional structure in this two-dimensional world) on top of the ridges become visible. Describing the landscape from the perspective of the peaks of density in Fig. 15 (c), the next-higher dimensional structures ("ridges") are features becoming discernible only at lower height of the density. In a cluster analysis, with $q_{\text {cut }}$ chosen too large, one might miss the ridge catching only the peaks. Varying $q_{\text {cut }}$ the dimension revealed by the cluster analysis will be very reminiscent of the multifractal dimensions of the individual fermionic modes. Compared to these visible features of the three different mode-truncated densities, the cut through the all-scale topological density is seemingly completely random. Nonetheless, structure can be discovered in the "full" density, too, simultaneously taking all four dimensions into account through the cluster algorithm, as we shall see next. 


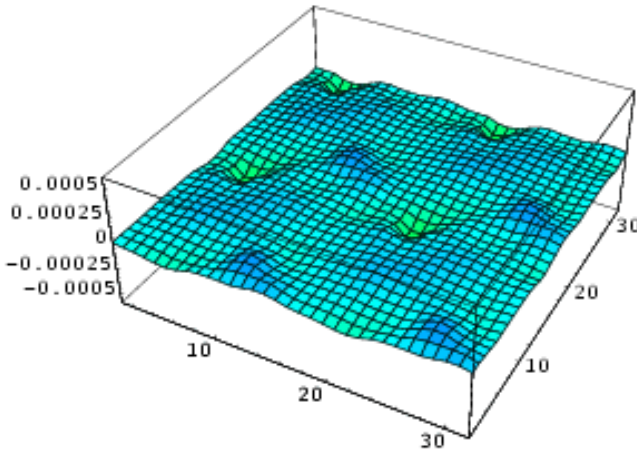

(a)

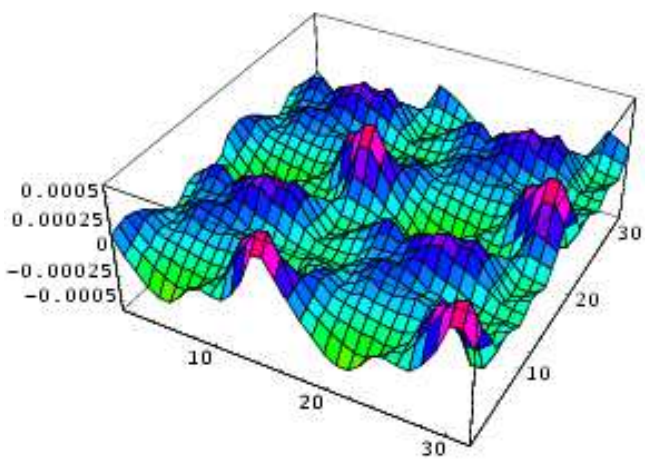

(c)

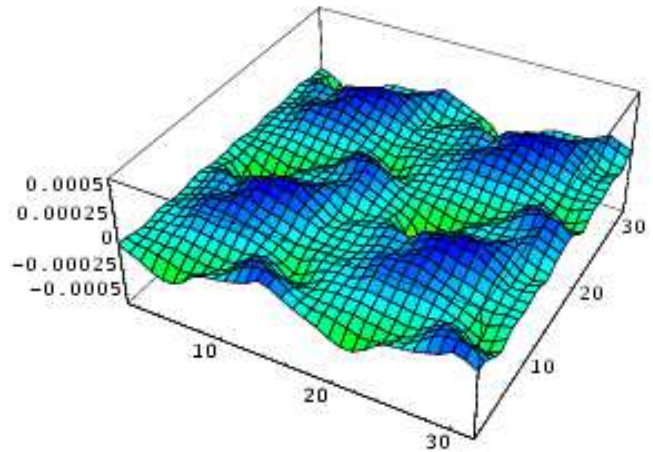

(b)

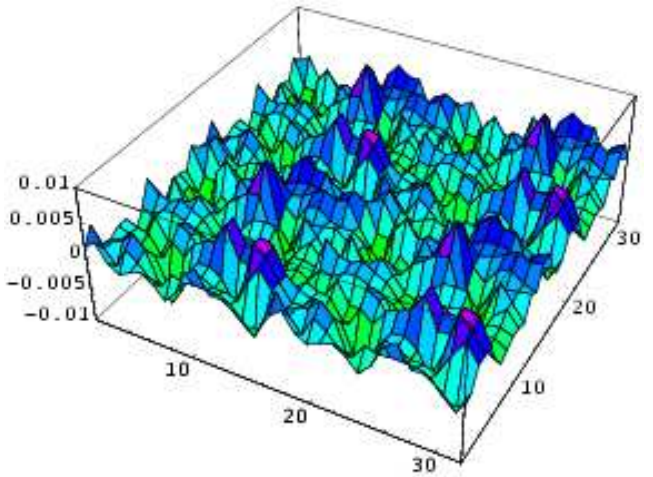

(d)

FIG. 15: Two-dimensional profile through the typical $Q=0$ configuration generated at $\beta=8.45$ on a $16^{3} \times 32$ lattice. The profile is periodically doubled in both directions for better visibility of extended structures. The cuts show, from (a) to (c), the mode-truncated density for descending levels of truncation $\lambda_{\text {cut }}=200 \mathrm{MeV}, \lambda_{\text {cut }}=400 \mathrm{MeV}$ and $\lambda_{\text {cut }}=634 \mathrm{MeV}$. The all-scale topological density is shown in $(d)$. Note the 20 -fold larger vertical scale in $(d)$.

\section{Cluster analysis of the all-scale topological charge density}

In order to make the structural analysis more quantitative, the cluster analysis as described in Section IIE is performed. Let us begin with the all-scale topological charge density. The structure is changing with the threshold value $q_{\text {cut }}$. As the reference value for $q_{\text {cut }}$ serves the maximum of the topological density in each configuration, $q_{\max }$. Thus, we take $0<q_{\text {cut }} / q_{\max }<1$ as the running parameter. We remark that $q_{\max }$ for the modetruncated density is weakly changing with $\lambda_{\text {cut }}$ (around 0.005 in lattice units). For the 


\begin{tabular}{|c|c|c|c|c|c|c|}
\hline$\lambda_{\text {cut }}$ & $200 \mathrm{MeV}$ & $400 \mathrm{MeV}$ & $600 \mathrm{MeV}$ & $634 \mathrm{MeV}$ & $800 \mathrm{MeV}$ & all-scales \\
\hline$\beta=8.10$ & $5.04(14) 10^{-3}$ & $6.45(14) 10^{-3}$ & $7.66(15) 10^{-3}$ & & $8.88(14) 10^{-3}$ & $2.82(07) 10^{-2}$ \\
$12^{3} \times 24$ & & & & & & \\
\hline$\beta=8.45$ & $3.51(17) 10^{-3}$ & $4.45(18) 10^{-3}$ & & $5.39(19) 10^{-3}$ & & $1.97(06) 10^{-2}$ \\
$16^{3} \times 32$ & & & & & & \\
\hline
\end{tabular}

TABLE IV: The ensemble averages of $q_{\max } a^{4}$ for the mode-truncated topological charge density (for various $\lambda_{\text {cut }}$ ) and for the all-scale density for two lattice ensembles.

all-scale topological density $q_{\max }$ is a few times larger (see Table IV).

In Fig. 16 the $q_{\text {cut }} / q_{\max }$ dependence of the cluster multiplicity, of the relative size of the largest cluster with respect to all clusters, of the distance between the two biggest clusters and of the connectivity are presented. This is shown for two ensembles of practically the same volume, $12^{3} \times 24$ at $\beta=8.10$ and $16^{3} \times 32$ at $\beta=8.45$, and, in order to study the $\beta$ dependence alone, for a few configurations of the same size $16^{3} \times 32$ in lattice units, but with a finer lattice spacing at $\beta=8.60$.

The most striking difference between the first two cases with almost equal physical volume is the roughly four times larger multiplicity of clusters that is reached on the finer lattice. In both lattices percolation (nonvanishing connectivity $f\left(r_{\max }\right)$ ) sets in close to where the multiplicity reaches its maximum. The percolation threshold $q_{\text {perc }}$ is practically independent of the lattice spacing. The percolation finally ends with $q_{\text {cut }} \rightarrow 0$ with only two, globally extended clusters of opposite sign of topological charge. With decreasing $q_{\text {cut }} / q_{\text {max }}$, but still above the percolation threshold at $q_{\text {cut }} / q_{\max }=0.2$, the number of clusters increases strongly with decreasing cut, in particular on the fine lattice. Since number and size of all clusters increase, the relative size of the biggest cluster decreases. This trend is reversed beyond the percolation threshold where the relative size of the largest cluster rapidly reaches $50 \%$, practically independent of the lattice spacing. The distance between the two biggest clusters shown in Fig. 16 (c) is given in lattice spacings. Before percolation the distance is therefore only seemingly bigger on the finer lattice. Taking the ratio $a(\beta=8.10) / a(\beta=8.45) \approx 1.4$ of the lattice spacings (see Table I) into account, the average distance between the two leading 


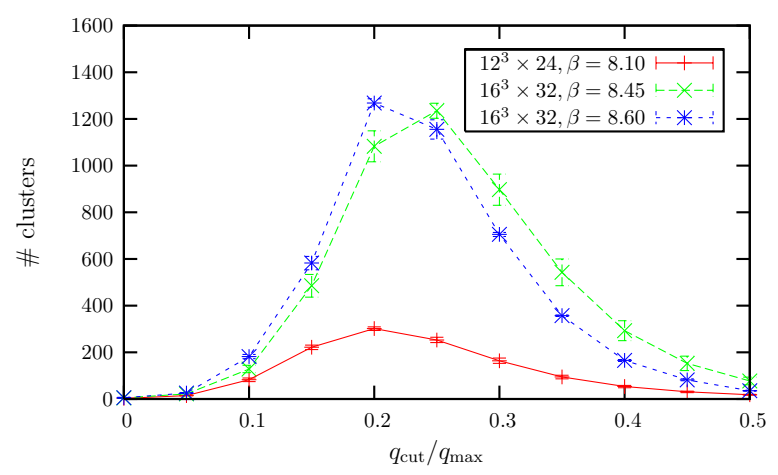

(a)

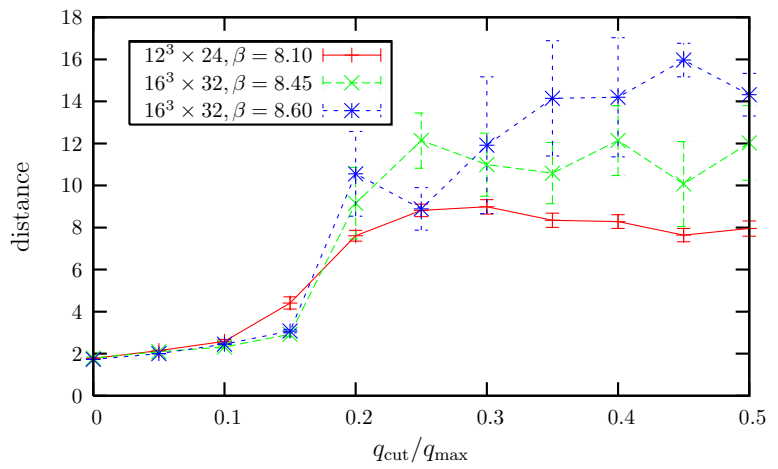

(c)

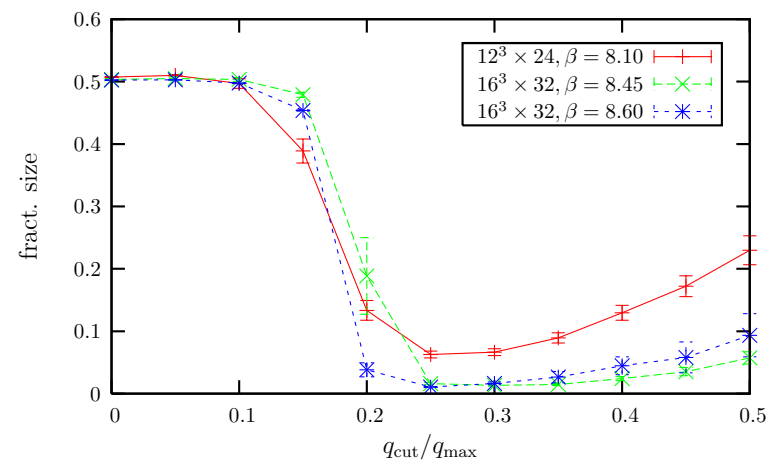

(b)

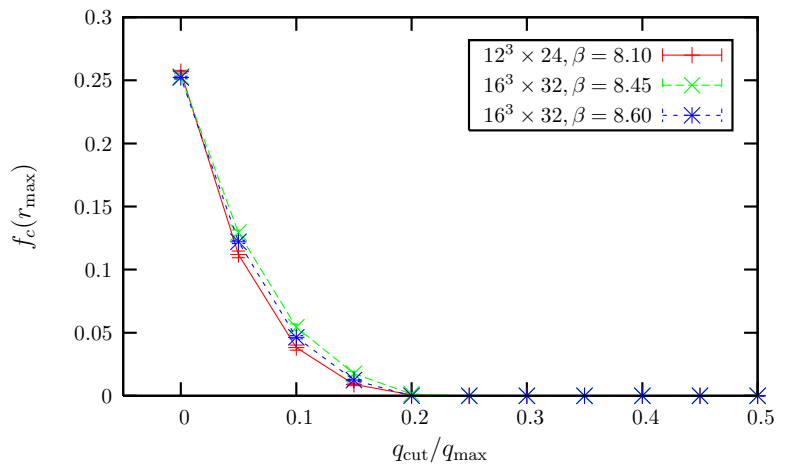

(d)

FIG. 16: Cluster analysis of the all-scale topological density: The $q_{\text {cut }}$ dependence is shown of (a) the total number of separate clusters, (b) the size of the largest cluster relative to all clusters, (c) the distance between the two largest clusters in lattice units and (d) the connectivity (see section IIC). The data is plotted both for the $12^{3} \times 24$ lattice configurations at $\beta=8.10$ (53 configurations, in red), for the $16^{3} \times 32$ lattice configurations at $\beta=8.45$ (only 5 configurations, in green) and for the $16^{3} \times 32$ lattice configurations at $\beta=8.60$ (only 2 configurations, in blue) being averaged over.

clusters is approximately the same and independent of $q_{\text {cut }} / q_{\max }$ all over the non-percolating regime. The finer the lattice, the faster the minimal distance between the two leading clusters is approaching the minimal distance of two lattice spacings as soon as $q_{\text {cut }}<q_{\text {perc }}$. In the final state the two clusters are close to each other everywhere, kept as separate clusters only because of the different sign of $q(x)$. In other words, the remaining two global clusters of opposite charge are everywhere thin of $O(a)$ and entangling each other. The finite distance in lattice spacings is independent of $\beta$ reflecting nothing else than the non-ultralocality of the overlap operator. 


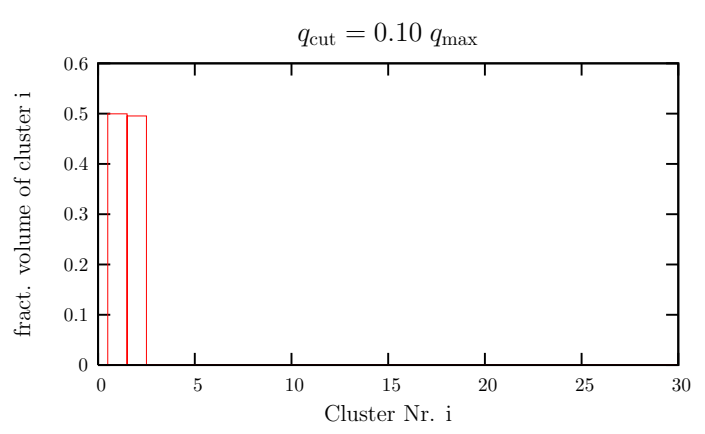

(a)

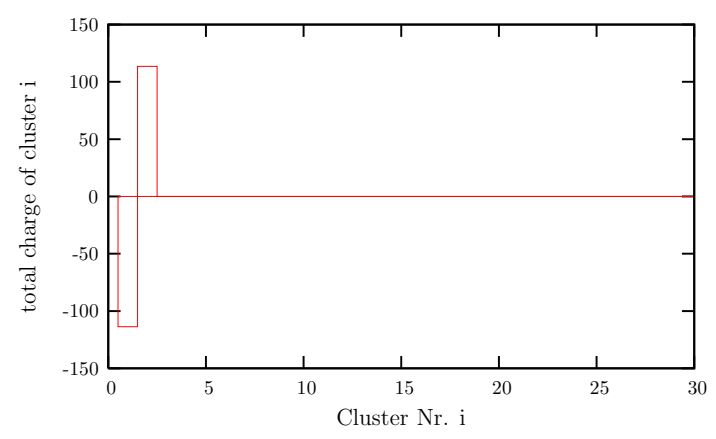

(c)

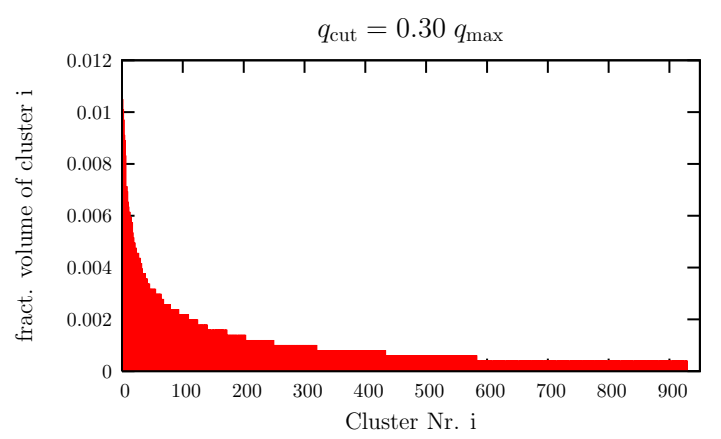

(b)

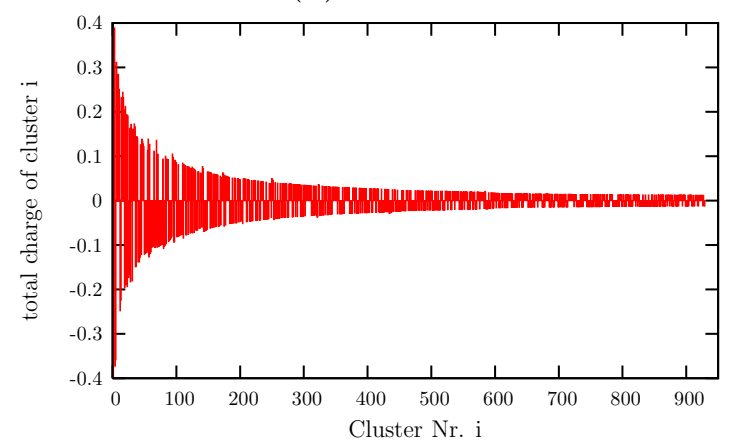

(d)

FIG. 17: Cluster analysis of the all-scale topological density: The fractional volume ((a)+(b)) and the total charge $((\mathrm{c})+(\mathrm{d}))$ of the corresponding clusters, ranked according to their occupied volume, is shown for two values of $q_{\text {cut }} / q_{\text {max }}$ : in the percolated regime (left) for $q_{\text {cut }} / q_{\max }=0.10$ and in the cluster-separated regime (right) for $q_{\mathrm{cut}} / q_{\mathrm{max}}=0.30$. The plot represents one single configuration of the $16^{3} \times 32$ lattice ensemble generated at $\beta=8.45$.

For the two lattices $16^{3} \times 32$ with different lattice spacing we find a surprising similarity in all four plots. Even the distance between the biggest clusters coincides within large errors due to the low statistics ( 5 and 2 configurations for $\beta=8.45$ and $\beta=8.60$, respectively). In particular, the cluster multiplicity seems to depend mostly on the lattice size in lattice units.

How the whole $q$-cluster composition with respect to charge and volume varies between the percolating regime $\left(q_{\text {cut }}=0.1 q_{\max }\right)$ and the non-percolating regime $\left(q_{\text {cut }}=0.3 q_{\max }\right)$, is illustrated in Fig. 17. As long as percolation has not set in, there is a broad spectrum of cluster volumes. The spectrum of cluster topological charges is surprisingly similar, up to the random sign. The ranking of clusters is according to the cluster volume, and the 


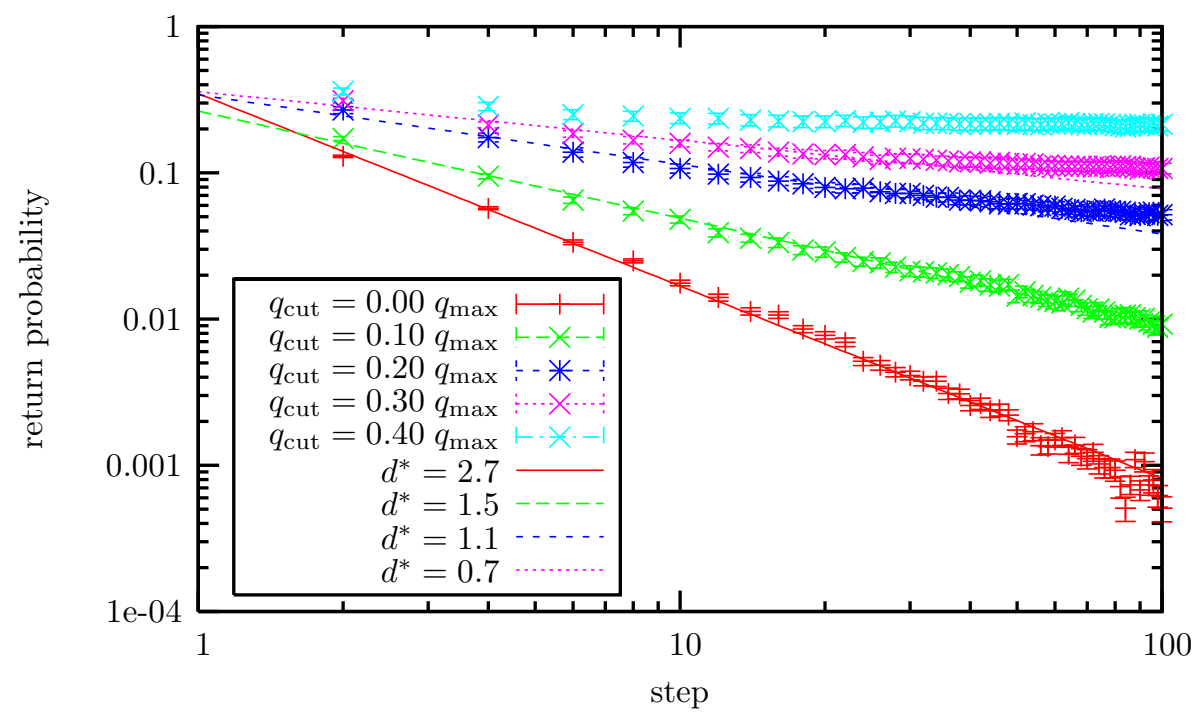

FIG. 18: The probability of return to the cluster center $x_{c}$ for random walks, restricted to topological clusters of the all-scale topological charge density, shown as a function of the step number. The clusters are distinguished by different cuts with respect to the density that they enclose. The cases $q_{\text {cut }} / q_{\max }=0.4,0.3,0.2,0.1$, and 0.0 are considered on the coarser lattice $12^{2} \times 24$ at $\beta=8.10$. The results of the power fits (see the curves) are labeled by the effective dimensions $d^{*}$.

modulus of the cluster charges almost follows this ranking. Thus, the average charge density in each cluster is approximately the same. When percolation is completed, there are only two, oppositely charged clusters left over with approximately equal volume. This peculiar situation has been first discovered and discussed by Horvath et al. [33].

\section{Dimensionality and multifractality of the all-scale topological charge}

In this subsection a more explicit description of the fractal dimension and the multifractal properties of this topological density will be given. These properties are difficult to visualize in three dimensions because of the close packing and the huge multiplicity (at the percolation threshold) of the corresponding $q$-clusters.

For our purpose we use the random walker method described in section IIF. In Fig. 18 we show in a double-logarithmic plot the decay of the return probability on the $12^{3} \times 24$ lattice. In an open $d^{*}$-dimensional space the return probability after $\tau$ steps would follow 
the power law $P(\overrightarrow{0}, \tau)=1 /(2 \pi \tau)^{d^{*} / 2}$. It turns out, that by extending the clusters $(i . e$. by chosing $q_{\text {cut }} / q_{\max }$ lower and lower) the power of decay becomes larger. The corresponding effective fractal dimensions are ranging from $d^{*}=2.7$ for low $q_{\text {cut }} / q_{\max } \approx 0$, over $d^{*}=1.5$ at $q_{\text {cut }} / q_{\max }=0.1$ and $d^{*}=1.1$ at $q_{\text {cut }} / q_{\max }=0.2$ where the $q$-cluster multiplicity reaches its maximum, to $d^{*}=0.7$ at $q_{\text {cut }} / q_{\max }=0.3$ where the clusters are really restricted to the immediate neighborhood of the maxima. For a survey of dimensionalities of clusters of the topological charge see Tables V and VI in the Appendix. Even if the values of the fractal dimensions $d^{*}$ provided by the random walker algorithm do not exactly coincide with the dimensions obtained from other methods, the tendency of the results of the random walk method indicates that the landscape of the all-scale topological charge is multifractal and becomes nearly three-dimensional only far below the percolation threshold. Percolation sets in at $q_{\text {cut }} \approx 0.25 q_{\text {max }}$, when the effective dimension is still low as $d^{*}=1-1.5$.

A second argument comes from a consideration of the cumulative cluster charge. One considers a collection of $q$-clusters primarily defined for a given cut-off $q_{\text {cut }} / q_{\max }$. One covers the set of clusters with a four-dimensional sphere of radius $R$ centered at the center $x_{c}$ of one particular cluster (i.e. the maximum of $|q(x)|$ inside the cluster $c$ ). One sums that part of the cluster charge that is located in the intersection of the cluster and the covering sphere. The relative cumulated cluster charge is the ratio between the cumulated charge to the full charge of the cluster and it depends on the radius $R$. The dependence of the relative cumulated cluster charge on the radius $R$ will start with some power and finally reach unity at saturation. The observed power behavior can give information about the fractal dimension $d^{*}<d$ of a $q$-cluster. The initial growth with $R$ may be parametrized as $R^{d^{*}}$, where $d^{*}$ describes the local dimension near the cluster center and depends on the cut-off $q_{\text {cut }} / q_{\text {max }}$. One can see in Fig. 19 that a linear fit works very well as long as $q_{\text {cut }} / q_{\max }$ is sufficiently high such that the clusters are isolated and do not percolate. The beginning percolation is characterized by an initial growth fitted like $Q_{\text {cumul }} \sim R^{3}$.

One might expect problems when $R$ is so large that the sphere covers many different threedimensional branches of a $q$-cluster which might appear disconnected inside the sphere but are actually globally connected (and therefore counted in the cumulative charge according to the cluster membership). This would happen at very low cut-off $q_{\text {cut }}$ when only the two largest clusters have survived. Only then the cumulated charge of each of the clusters would 


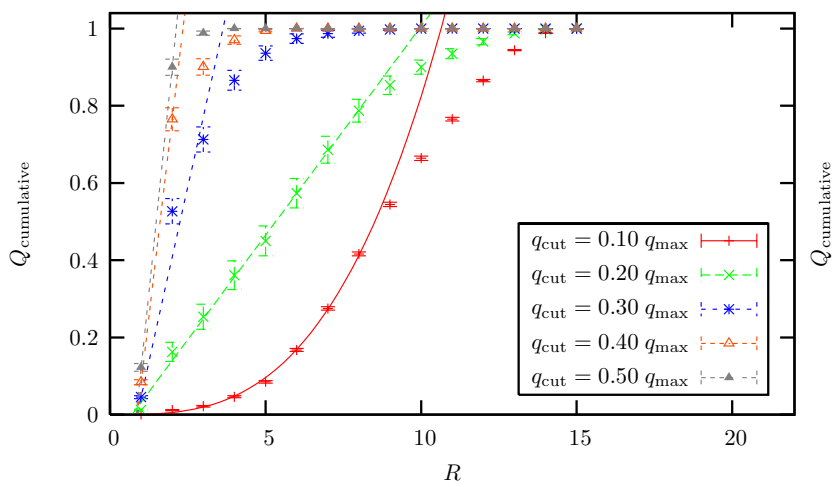

(a)

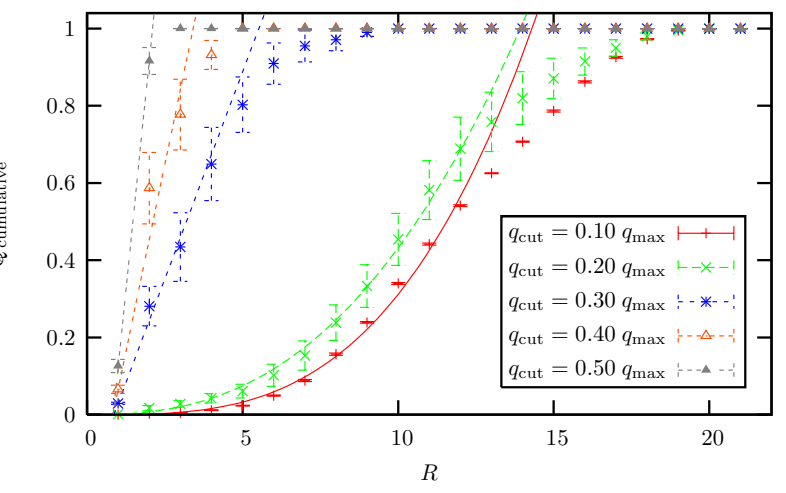

(b)

FIG. 19: The covering sphere method (see text): (a) for the coarse lattice $12^{3} \times 24$ at $\beta=8.10$, (b) for the fine lattice $16^{3} \times 32$ at $\beta=8.45$. The fitted power behavior of the accumulated charge jumps from one-dimensional to three-dimensional at some percolation threshold $q_{\text {cut }} / q_{\text {max }}$ (see text).

grow like $Q_{\text {cumulative }} \sim R^{4}$ although the local dimension is $d^{*}=3{ }^{5}$.

One can see in Fig. 19 that the transition from linear to cubic behavior happens for the coarser lattice (left panel) somewhere between $q_{\text {cut }} / q_{\max }=0.1$ and 0.2 . The actual power behavior for the lowest shown cut-off is like $Q_{\text {cumulative }} \sim R^{3.1}$. On the finer lattice, for the higher cut-off $q_{\text {cut }} / q_{\max }=0.2$ the situation is already close to the $d^{*}=3$ regime. The fitted power behavior is like $Q_{\text {cumulative }} \sim R^{2.5}$. For $q_{\text {cut }} / q_{\text {max }}=0.1$ it is like $R^{3.3}$.

For a survey of dimensionalities of the topological density (as well as of overlap eigenmodes) inferred from the covering sphere method see Table VI (and Table VIII) in the Appendix.

\section{E. Cluster analysis of the mode-truncated topological charge density}

Let us now discuss the effect of mode-truncation on the cluster structure of the topological charge density. We compare in Fig. 20 for the two ensembles, $12^{3} \times 24$ at $\beta=8.10$ (left) and $16^{3} \times 32$ at $\beta=8.45$ (right) with practically equal physical volume, the ensemble averages of the cluster multiplicity, of the fractional volume filled by all clusters (packing fraction)

\footnotetext{
5 This consideration was the motivation to propose the random walker method described in Section $\coprod$

The walkers are not allowed to penetrate to or return from another branch of the same cluster.
} 


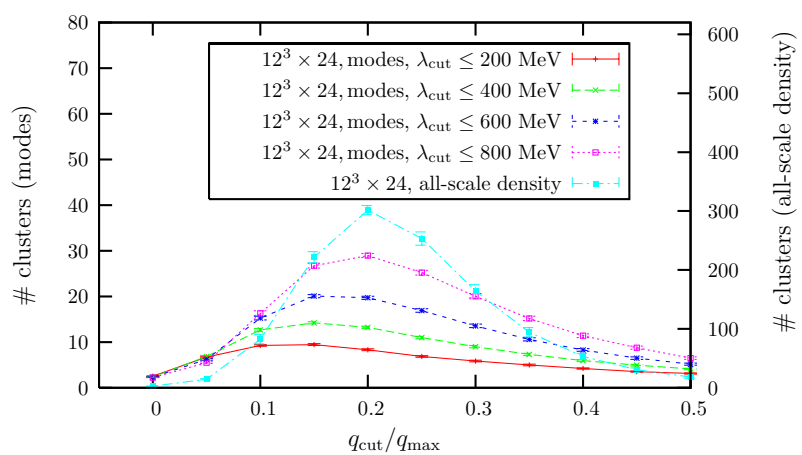

(a)

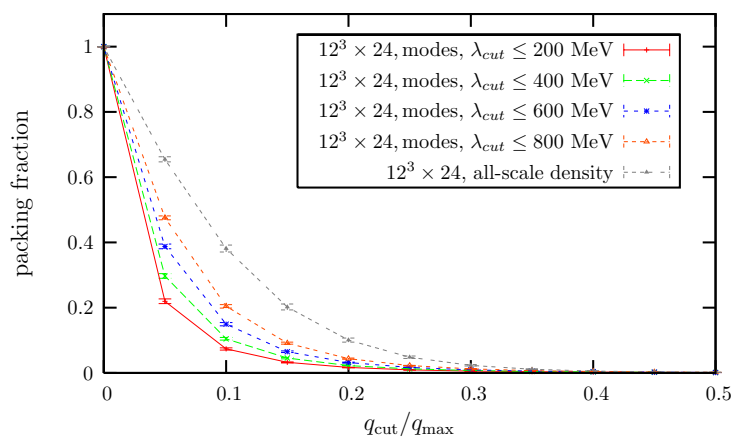

(c)

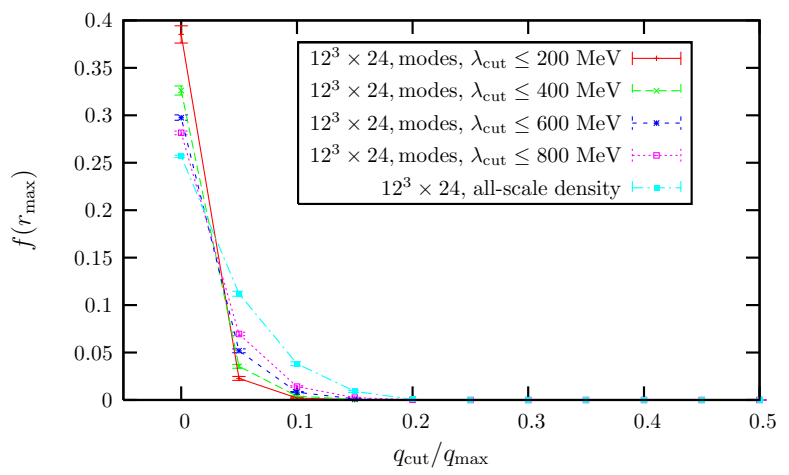

(e)

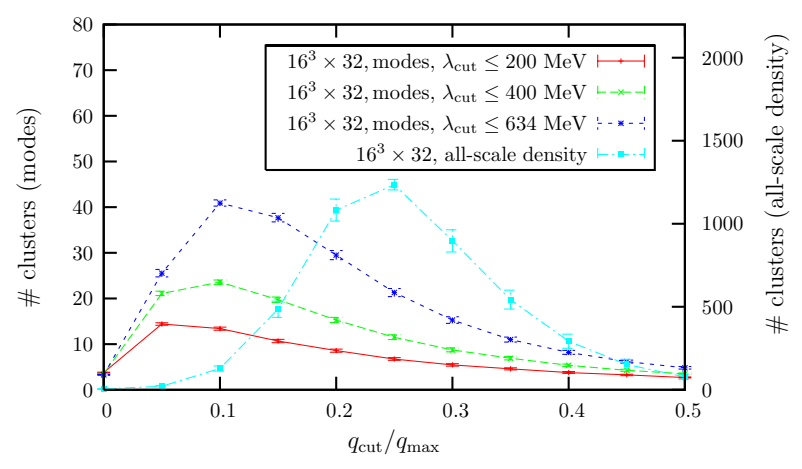

(b)

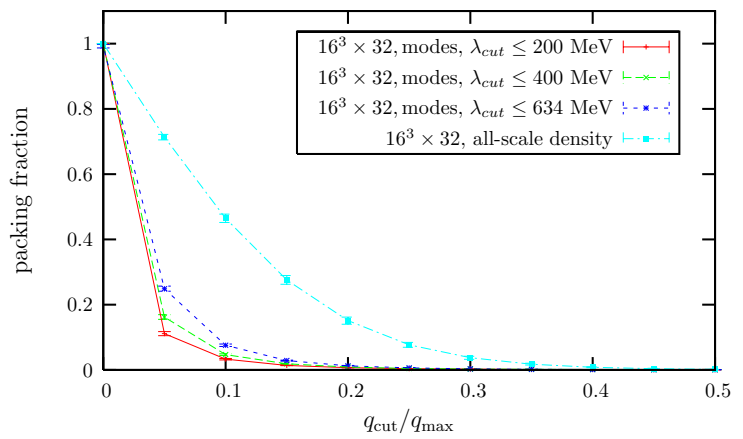

(d)

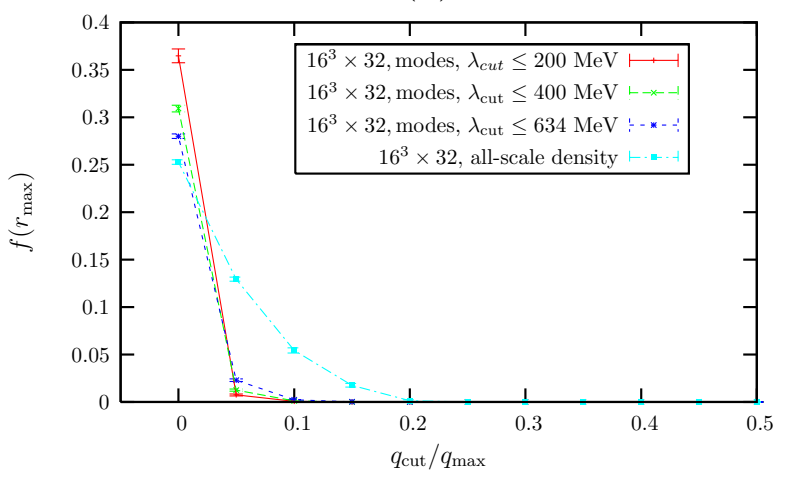

(f)

FIG. 20: Comparison of the cluster structure of the mode-truncated density with three resp. four cut-offs $\lambda_{\text {cut }}$ with the all-scale topological charge density. The effect of $\beta$ at the same physical volume can be seen by comparing the left side $\left(12^{3} \times 24\right.$ lattices at $\left.\beta=8.10\right)$ with the right side $\left(16^{3} \times 32\right.$ lattices at $\left.\beta=8.45\right)$. Top row: the $q_{\text {cut }}$ dependence of the total number of clusters $((\mathrm{a})$ and (b)). Note the different multiplicity scale on the right for clusters of the all-scale topological charge density. Middle row: the packing fraction of all clusters together ((c) and (d)). Bottom row: the connectivity ((e) and (f)). 


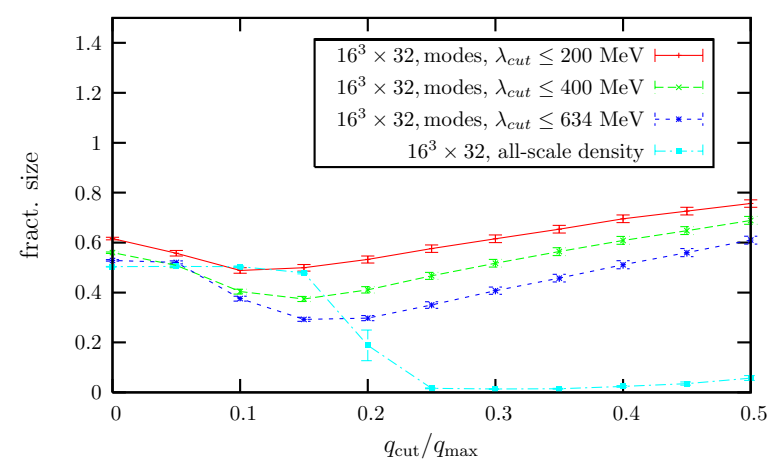

(a)

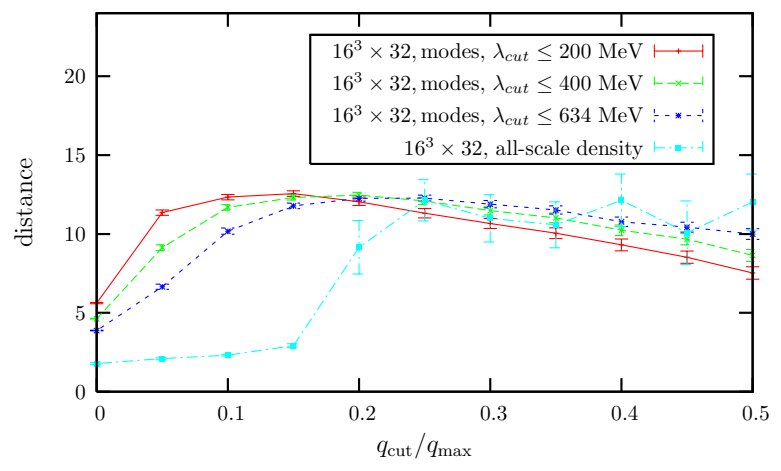

(b)

FIG. 21: Comparison of the cluster structure of the all-scale topological charge density with the mode-truncated density for three values of the cut-offs $\lambda_{\text {cut }}$ : (a) the fractional size of the largest cluster and (b) the distance between the two largest clusters in lattice units, both as function of $q_{\text {cut }} / q_{\text {max }}$. The data refers to the $16^{3} \times 32$ lattice at $\beta=8.45$ only.

and of the connectivity $f\left(r_{\max }\right)$. In each panel we show this for 3 or 4 cut-offs $\lambda_{\text {cut }}$ together with the same quantity defined for the all-scale density. Notice the different scale for the cluster multiplicity for the all-scale density on the right of the upper panels. For the coarser lattice presented on the left and the finer lattice presented on the right, the tendency is the same. The maximal number of clusters is much smaller for the mode-truncated density than for the all-scale density. It rises with the cut-off $\lambda_{\text {cut }}$. For a fixed cut-off $\lambda_{\text {cut }}$ in MeV, it is approximately 40 to $100 \%$ larger on the finer lattice than on the coarser lattice. Thus, the cluster multiplicity defined with a fixed ultraviolet cut-off $\lambda_{\text {cut }}$, is strongly discretization dependent. The maximum of cluster multiplicity is reached at ever smaller $q_{\text {cut }} / q_{\max }$ for the finer lattice. The packing fraction, i.e. the fraction of volume filled by the clusters, for the all-scale density rises faster with decreasing $q_{\text {cut }}$ on the finer lattice than on the coarser. On the other side, for the mode-truncated densities, the final rise of the packing fraction happens almost simultaneously for $q_{\text {cut }}<0.1 q_{\text {max }}$ for all cut-offs $\lambda_{\text {cut }}$ under consideration. This means that, as long as $q_{\text {cut }} / q_{\max }>0.1$, the clusters remain well separated. These differences are washed out on the coarser lattice. On the finer lattice the maximal cluster multiplicity (or the percolation threshold) for the all-scale density is found at much higher $q_{\text {cut }} / q_{\max }$ value than for the mode-truncated density including the highest investigated $\lambda_{\text {cut }}=634 \mathrm{MeV}$. On the coarser lattice this happens to the all-scale and the mode-truncated density at the same $q_{\text {cut }} / q_{\text {max }}$. 
For the finer lattice only, in Fig. 21 the relative size of the largest cluster and the distance between the two largest clusters is plotted in dependence on $q_{\text {cut }} / q_{\text {max }}$. Each panel shows this for various $\lambda_{\text {cut }}$ and for the all-scale density. The behavior for the different cut-offs $\lambda_{\text {cut }}$ of mode-truncated densities is similar. The main difference of the mode-truncated density compared to the non-truncated (all-scale) density is that the relative size of the largest cluster remains relatively constant before and across the percolation threshold although the number and size of all clusters increases with decreasing $q_{\text {cut }}$ before percolation sets in. The largest cluster is always much bigger than the other clusters, and this effect is the stronger the lower the cut-off $\lambda_{\text {cut }}$ is. It can be clearly seen that the distance $d(a, b)$ between the two largest clusters $a$ and $b$ of the mode-truncated density does not drop rapidly to 2 lattice spacings immediately after the onset of percolation. This is because these clusters remain well-separated even at $q_{\text {cut }} / q_{\max }<0.1$.

All these observations point towards the conclusion that the mode-truncated density allows to define $q$-clusters of topological charge that, although they also begin to percolate at some (relatively low) height (cut-off $q_{\text {cut }}$ ), remain well-separated even at this level of the density. One of the clusters is much larger than the remaining ones. These properties they have in common with the $R$-clusters that will be defined on the basis of the local (anti-)selfduality $R(x)$ in Section $\mathrm{VC}$. Thus, these clusters allow an interpretation close to the traditional "lumpy" instanton or caloron gas picture, however with the tendency to coalesce into larger objects of same-sign topological charge density ("instanton clumping"). At higher $\lambda_{\text {cut }}$ an attractive correlation between opposite charge clusters appears.

Given this cluster interpretation of the mode-truncated topological density, we return to the question whether the chirality $X_{n}(x)$ of the $n$-th non-zero mode is locally correlated with the topological charge density. To answer this question we consider the correlation function between the density $q_{\lambda_{\text {cut }}}(y)$ and the local chirality $X(y)$ over some distance $r, C_{q X}\left(r ; \lambda_{\text {cut }}, n\right)$ defined as

$$
C_{q X}\left(r ; \lambda_{\text {cut }}, n\right)=\frac{\sum_{x, y}\left\langle q_{\lambda_{\text {cut }}}(x) X_{n}(y)\right\rangle \delta(r-|x-y|)}{\sum_{x, y} \delta(r-|x-y|)} .
$$

The result is shown in Fig. 22 for $\lambda_{\text {cut }}=100 \mathrm{MeV}$. The local chirality that enters the correlator in the figure is evaluated for the $2 \mathrm{nd}$, the 6 th, the $10 \mathrm{th}$, the 30 th, the 50 th etc. up to the 120th non-zero mode. We keep in mind that this mode-restricted topological charge density is influenced (on average) by the lowest 9 or 10 pairs of non-zero modes. 


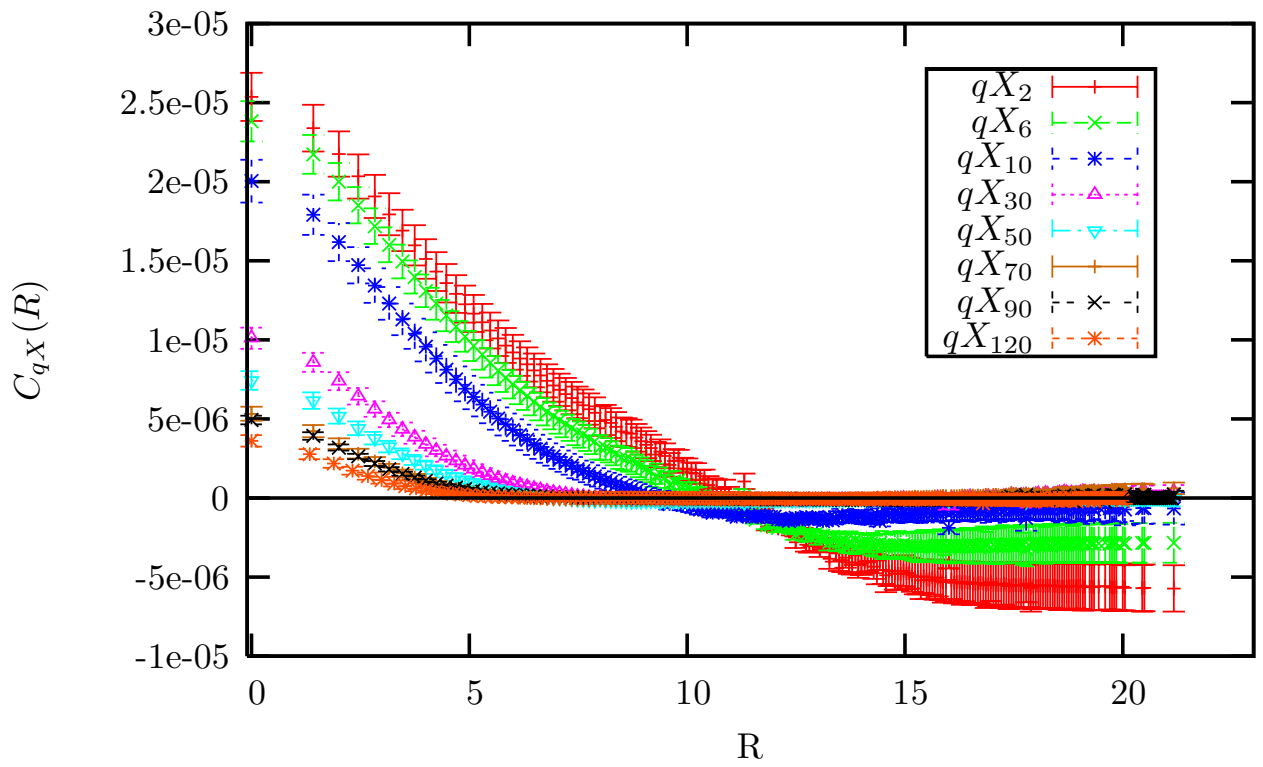

FIG. 22: The correlation function between the mode-truncated topological charge density for $\lambda_{\text {cut }}=100 \mathrm{MeV}$ and the local chirality of selected non-zero modes from the lowest one to the 120th mode. The correlation function is an average over the 37 configurations with $Q=0$ on the $16^{3} \times 32$ lattice generated at $\beta=8.45$.

We see that the positive correlation of the (collective) topological charge density on one hand with the local chirality of individual overlap modes up to the 10th mode on the other hand is strong and ranges over up to ten lattice spacings (similar to the distance where the correlation function $C_{q q}$ of $q_{\lambda_{\text {cut }}}$ changes the sign. At larger distance the local chirality gets anticorrelated to the mode-truncated topological charge density. Although weaker and over shorter distance, the 30th and higher modes are still positively correlated with the sign of the truncated topological charge density. For these higher modes the anticorrelation is absent at larger distance.

\section{THE INFRARED FIELD STRENGTH TENSOR}

\section{A. Basic idea}

We have considered in Section IV the topological charge density as represented by the overlap operator according to Eqs. (18) or (19). We have seen that the structure of the mode- 
truncated topological charge density strongly depends on the number of included lowest eigenmodes. Restricting to fewer modes is tantamount to an ultraviolet filtering applied to the topological density. Our point of view as explained in Section \1 is that the low-lying modes represent the physically relevant degrees of freedom for hadronic physics. Thus, filtering should be suitable to highlight the important structures. Here we briefly discuss another filtering method similar to what was proposed by Gattringer [38] and is suggested by the newly discussed representation of the field strength tensor through the overlap Dirac operator [39, 40]. The main purpose is to get an ultraviolet filtered, infrared field strength tensor. This would allow to assess the local degree of (anti-)selfduality. The local selfduality or antiselfduality of a gauge field is an important feature in all semiclassically motivated vacuum models, and the search for it in generic lattice configurations may play a similar (or superior) role compared to the search for lumps of topological charge, which was the main strategy of access before.

The starting point is the observation that in the continuum the square of the Dirac operator $D$, projected with the help of a combination of $\gamma_{\mu}$ matrices, represents the field strength tensor

$$
F_{\mu \nu}(x)=-\frac{1}{4} \operatorname{tr}_{\text {Dirac }}\left(\sigma_{\mu \nu} D^{2}(x)\right) .
$$

Here $\operatorname{tr}_{\text {Dirac }}$ is the trace taken over spinor indices. In Ref. [38] the chirally improved Dirac operator [84] was used to evaluate this in the simplified local form

$$
\begin{aligned}
F_{\mu \nu}^{a}(x) & \propto \sum_{\lambda} \lambda^{2} f_{\mu \nu}^{a}(x, x \mid \lambda), \\
f_{\mu \nu}^{a}(x, x \mid \lambda) & =-\frac{i}{2} \psi_{\lambda}^{\sigma c *}(x) \sigma_{\mu \nu}^{\sigma} \sigma^{\prime} T_{c c^{\prime}}^{a} \psi_{\lambda}^{\sigma^{\prime} c^{\prime}}(x)
\end{aligned}
$$

with the color generator $T^{a}$ in the fundamental representation. Zero modes do not contribute to this representation of the field strength. The ultraviolet filtering consists now in including only a certain number of low-lying modes in this spectral representation.

Here we explore the application of this formula to the eigenmodes of the overlap Dirac operator $D(0)$ and compare the revealed structure with that shown by the ultraviolet filtered topological density. Obviously, a normalization factor remains undetermined, in particular because only a subset of low-lying modes will be included in the filter.

In two recent papers [39, 40], a representation of field strength and action density has 


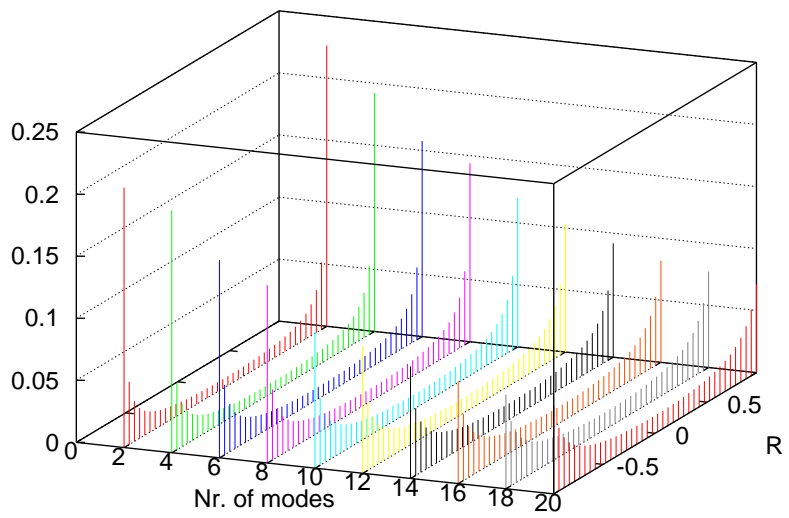

(a)

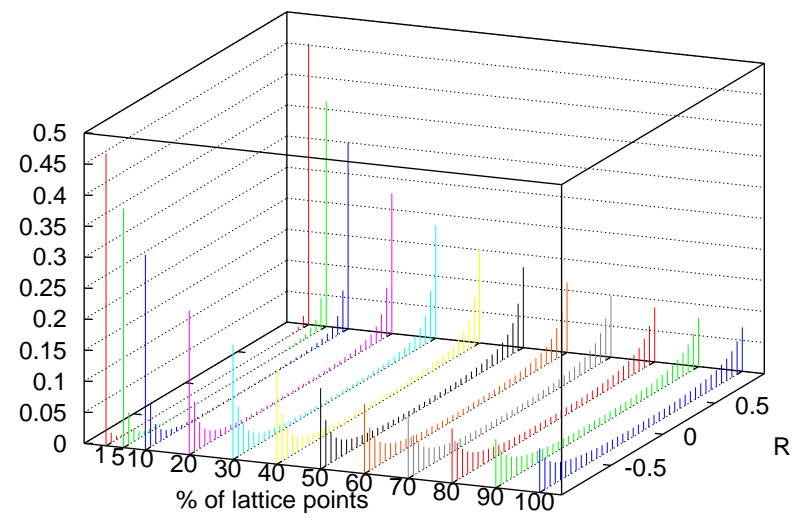

(b)

FIG. 23: Normalized histograms with respect to the local (anti-)selfduality of the field strength tensor in the $Q=0$ subsample consisting of 37 configurations generated on the $16^{3} \times 32$ lattice at $\beta=8.45$, (a) taken over $100 \%$ of the lattice sites, but depending on the number of non-zero modes (2 - 20) included in the filter, (b) applying various cuts specifying the infrared action density for the case of 20 eigenmodes included in the filter.

been discussed, in a way analogous to Eqs. (18) and (19), which give the representation of the topological charge density directly in terms of the overlap operator.

\section{B. Local (anti-)selfduality of the infrared field strength tensor}

We now study the contribution of individual non-zero modes to the filtered field strength tensor. The normalization of the infrared field strength $F_{\mu \nu}^{a}(x)$ does not come automatically. It could be obtained requiring that the infrared topological charge density

$$
\tilde{q}_{\mathrm{IR}}(x) \propto \operatorname{Tr}\left(F_{\mu \nu}(x) \tilde{F}_{\mu \nu}(x)\right)
$$

with the dual field strength $\tilde{F}_{\mu \nu}=\frac{1}{2} \epsilon_{\mu \nu \rho \sigma} F_{\rho \sigma}$ fits the ultraviolet filtered topological density $q(x)_{\lambda_{\text {cut }}}$ (for a suitable cut-off $\lambda_{\text {cut }}$ ). The latter can be directly derived from the overlap operator following Eq. (19). A less elaborate normalization for $Q \neq 0$ configurations could be that the total topological charge obtained from $\operatorname{Tr}\left(F_{\mu \nu}(x) \tilde{F}_{\mu \nu}(x)\right)$ should reproduce the 
index $Q$ of $D(0)$ for the given configuration.

One can define analogously the infrared action density

$$
\tilde{s}_{\mathrm{IR}}(x) \propto \operatorname{Tr}\left(F_{\mu \nu}(x) F_{\mu \nu}(x)\right)
$$

in order to compare it with $\tilde{q}_{\mathrm{IR}}(x)$. With both infrared densities at hand, one can ignore the quest for normalization. It is now possible to turn the interest to the local (anti-)selfduality based on a ratio similar to Eq. (30),

$$
r(x)=\frac{\tilde{s}_{\mathrm{IR}}(x)-\tilde{q}_{\mathrm{IR}}(x)}{\tilde{s}_{\mathrm{IR}}(x)+\tilde{q}_{\mathrm{IR}}(x)} .
$$

This is converted to $R(x)$,

$$
R(x)=\frac{4}{\pi} \arctan (\sqrt{r(x)})-1 \in[-1,+1],
$$

analogously to Eq. (31).

In Fig. 23 histograms are presented with respect to $R(x)$ applying various cuts specifying the local action density $\tilde{s}_{\mathrm{IR}}(x)$. Firstly, sharp peaks at $R= \pm 1$ are observed that become weaker with the inclusion of more low-lying pairs of non-zero modes. We notice that even with 10 pairs included these peaks are still visible. They become more pronounced again when one focusses on part of the lattice sites applying a cut with respect to the infrared action density $\tilde{s}_{\mathrm{IR}}(x)$.

\section{Cluster analysis of selfdual and antiselfdual domains}

Since the close neighborhood of $R= \pm 1$ of these histograms corresponds to lattice points where the infrared field strength tensor is nearly (anti-)selfdual, it is interesting to see whether they are completely disconnected or form connected regions in space-time. We construct isosurface plots of $|R(x)|=R_{\text {cut }}=1-\varepsilon$ demonstrated in Fig. 24 for a filter including the lowest 5 pairs of non-zero modes. The bubbles denote regions where the infrared field strength tensor is more (anti-)selfdual inside than outside. Red refers to antiselfdual regions, green to selfdual ones. Similar clusters have been seen when we have plotted clusters of the ultraviolet filtered topological charge density.

In order to get a more quantitative characterization of the vacuum, it is useful to perform a cluster analysis on the basis of the quantity $R(x)$. In Fig. 25 we show how the cluster 


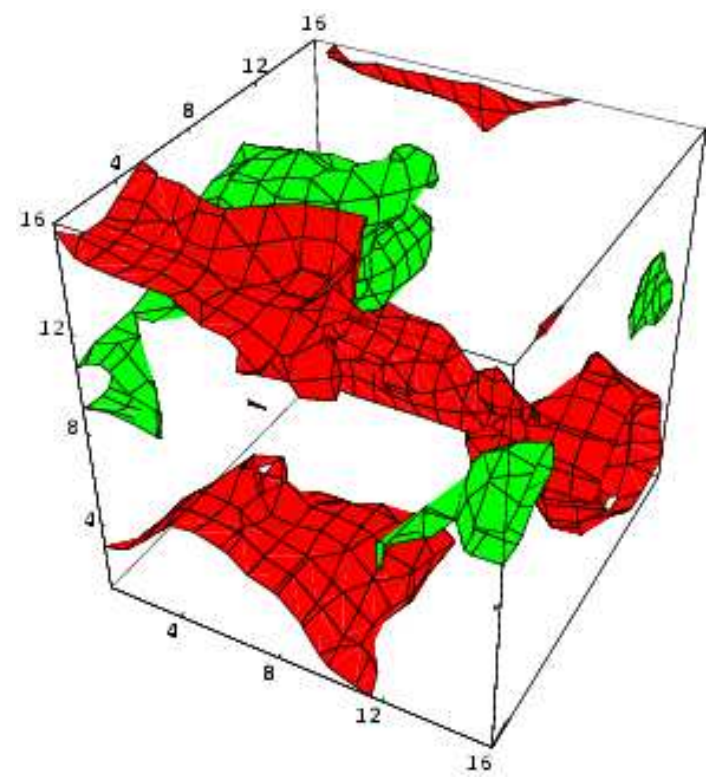

(a)

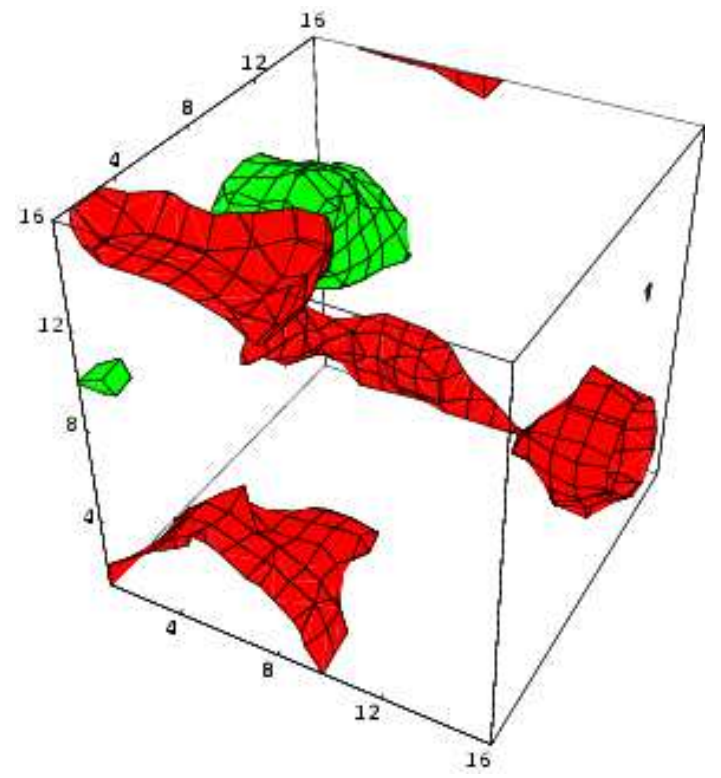

(c)

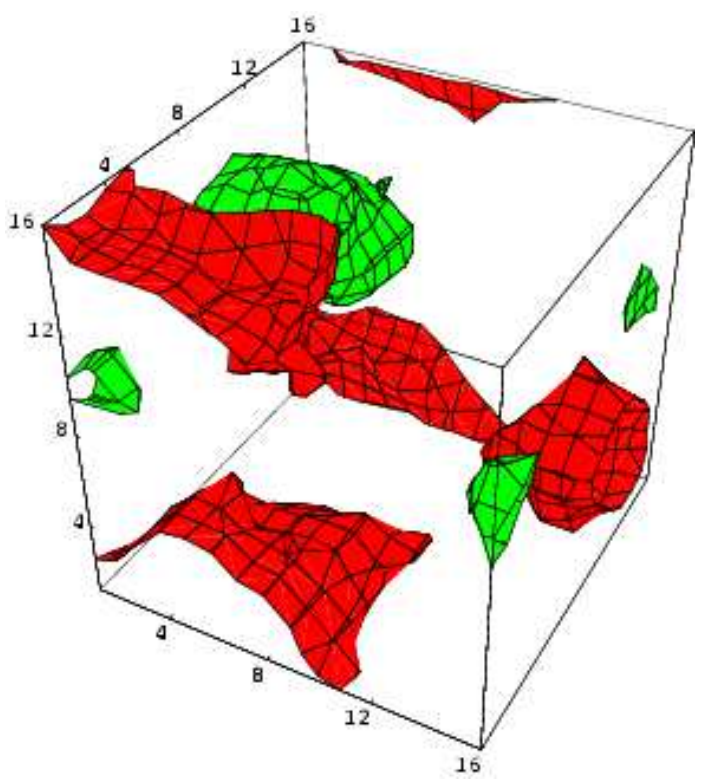

(b)

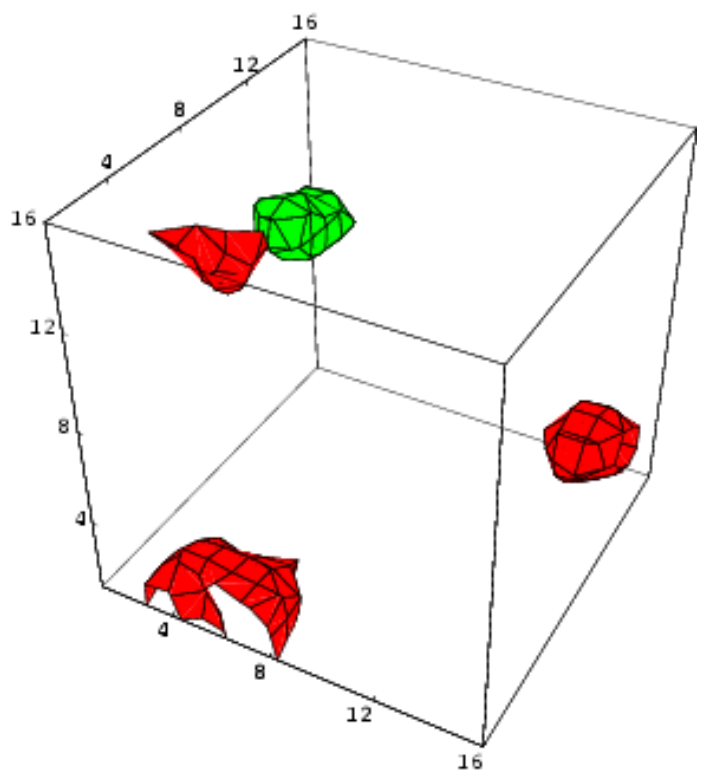

(d)

FIG. 24: Isosurface plots of $R(x)$ in the order of decreasing tolerance with respect to deviations from perfect (anti-)selfduality, (a) $R_{\text {cut }}=0.97$, (b) $R_{\text {cut }}=0.98$, (c) $R_{\text {cut }}=0.99$ and (d) $R_{\text {cut }}=0.999$. The figure shows the timeslice $t=6$ of the typical $Q=0$ configuration on the $16^{3} \times 32$ lattice generated at $\beta=8.45$. Red and green surfaces enclose regions where antiselfduality or selfduality is better fulfilled than in the rest of the volume. The filter is using the 5 lowest pairs of non-zero eigenmodes. 


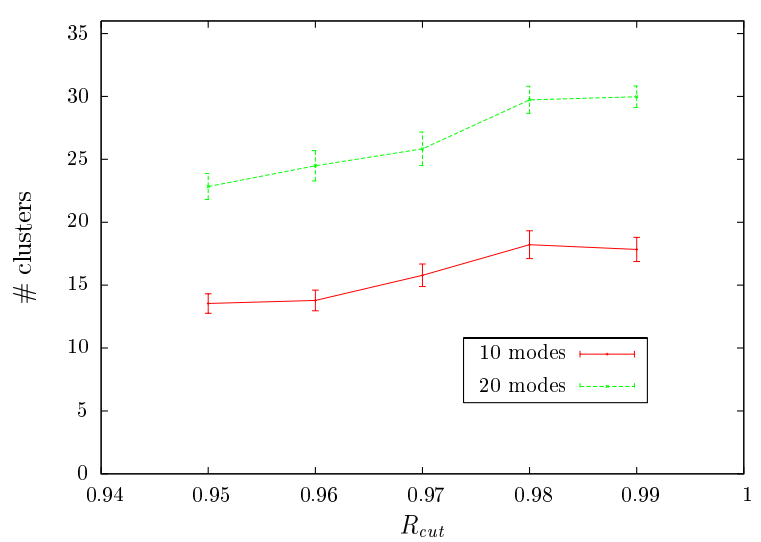

(a)

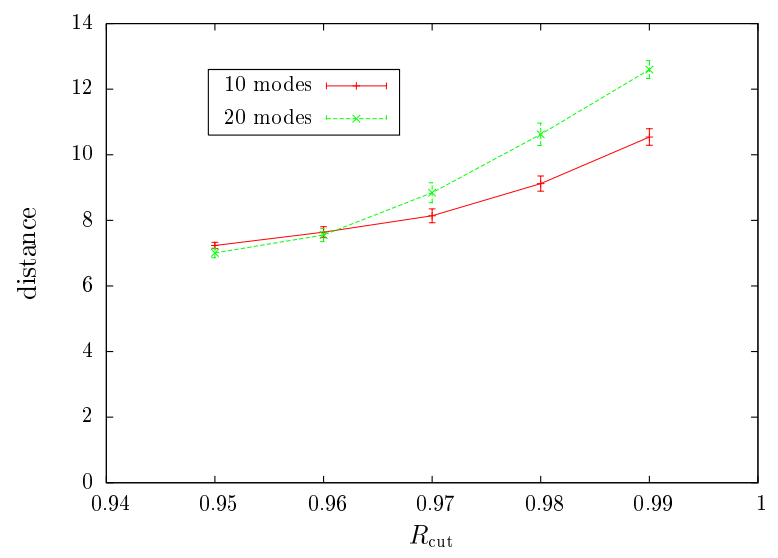

(c)

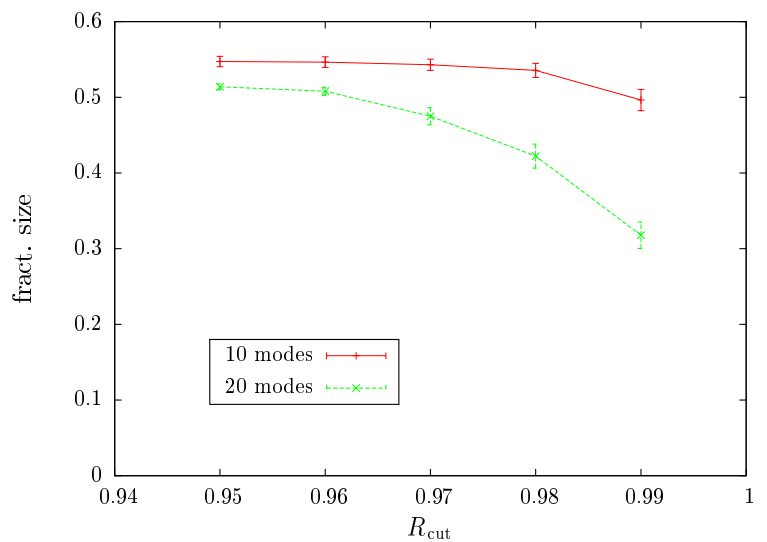

(b)

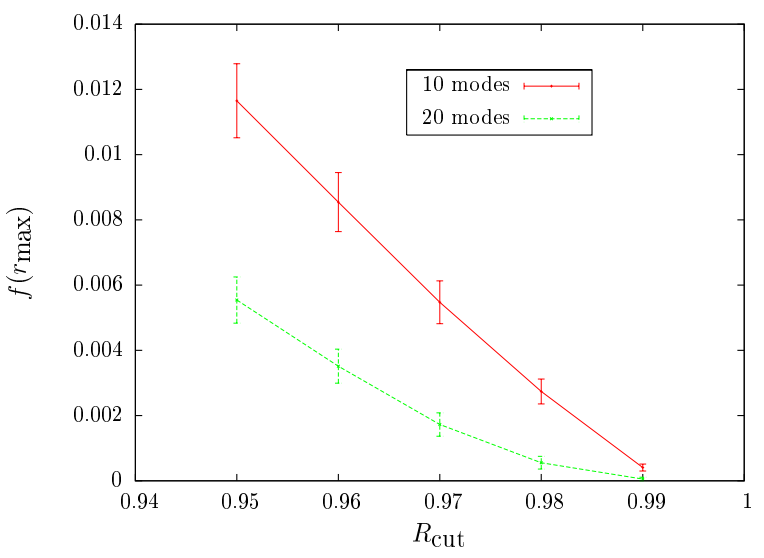

(d)

FIG. 25: $R$-cluster analysis using a running cut-off $R_{\text {cut }}$ on the basis of the filtered action density $s_{\text {IR }}$ and topological charge density $q_{\text {IR }}$ used to define $R(x)$. The $R_{\text {cut }}$ dependence is shown of (a) the total number of separate clusters, (b) the size of the largest cluster relative to all clusters, (c) the distance between the two largest clusters and (d) the connectivity (see text). The data is averaged over the $16^{3} \times 32$ lattice ensemble generated at $\beta=8.45$.

composition changes with varying $R_{\text {cut }}$. When percolation begins close to $R_{\text {cut }}=0.98$ the number of separate $R$-clusters starts to slightly decrease with decreasing $R_{\text {cut }}$. This means that allowing more tolerance with respect to violation of (anti-)selfduality inside the clusters leads to the growth and coalescence of already identified clusters, rather than to the detection of further, isolated clusters. One can see that the distance between the two largest clusters remains relatively large (decreasing only from 12 to 7 ), meaning that a lot of space-time remains unoccupied by the almost (anti-)selfdual $R$-clusters, even in the percolating regime signaled by the connectivity becoming $f\left(r_{\max }\right) \neq 0$. The unoccupied regions are regions 


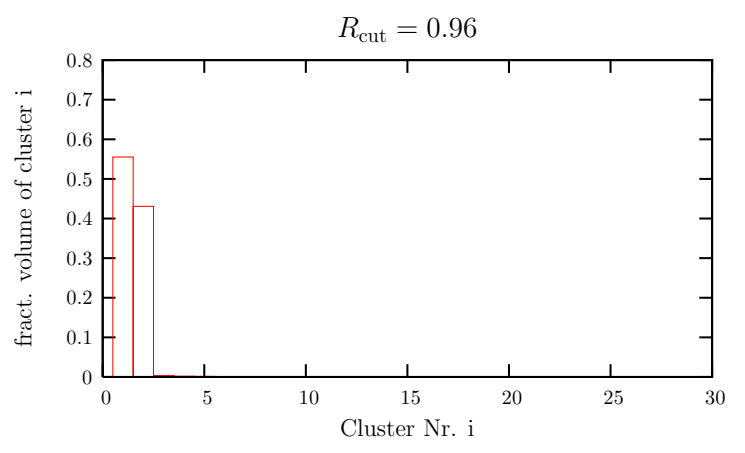

(a)

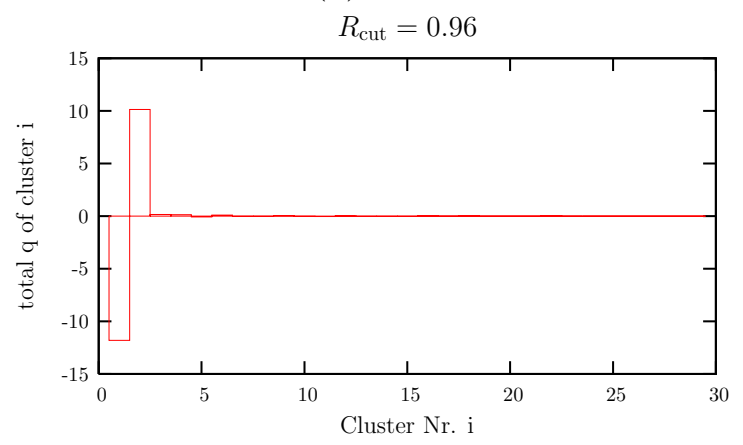

(c)

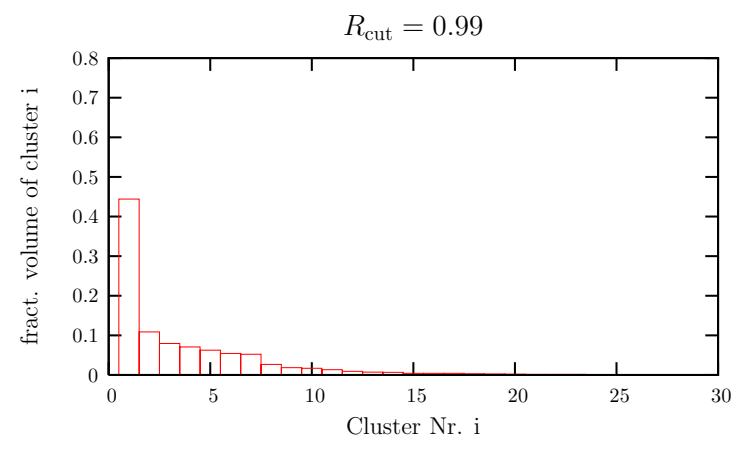

(b)

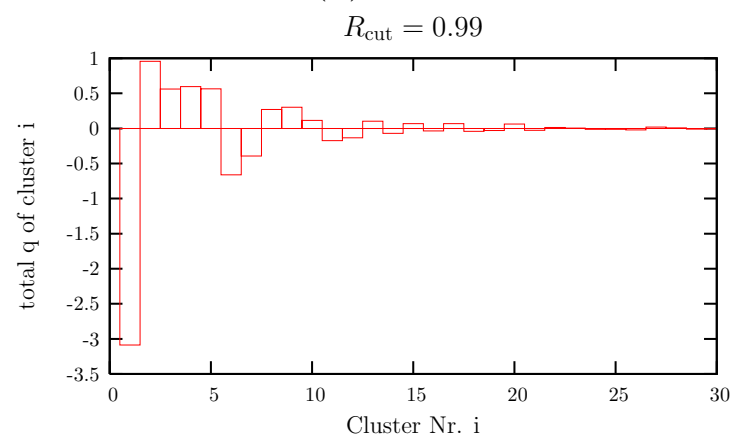

(d)

FIG. 26: Results of the $R$-cluster analysis at $R_{\text {cut }}=0.96$ (left) and $R_{\text {cut }}=0.99$ (right) concerning the relative volume of all emerging clusters ((a) and (b)) and concerning the charge inside the clusters $((\mathrm{c})$ and $(\mathrm{d}))$. The cluster charge is defined by the all-scale topological charge density (18). The distributions describe the same $Q=0$ configuration from the $16^{3} \times 32$ lattice ensemble generated at $\beta=8.45$ already considered in Fig. 6 and 24,

where the infrared field strength tensor is violating selfduality or antiselfduality more than the given tolerance $R_{\text {cut }}$ permits.

It turns out again that one largest cluster exists, which is rather large already before the onset of percolation, occupying $40 \%$ of the total volume of all clusters. This $R$-cluster rapidly becomes the absolutely dominating one with respect to the volume (more than 50 \%) after percolation has set in. This fact (topological "clumping") remains unexplained in all instanton/caloron models which are based on uncorrelated (anti-)selfdual objects of $Q= \pm 1$, eventually taking the attractive correlation of opposite charge cluster into account (that is actually seen at higher resolution $\lambda_{\text {cut }}$ ).

How the whole cluster collection changes between the percolating $\left(R_{\text {cut }}=0.96\right)$ and the 


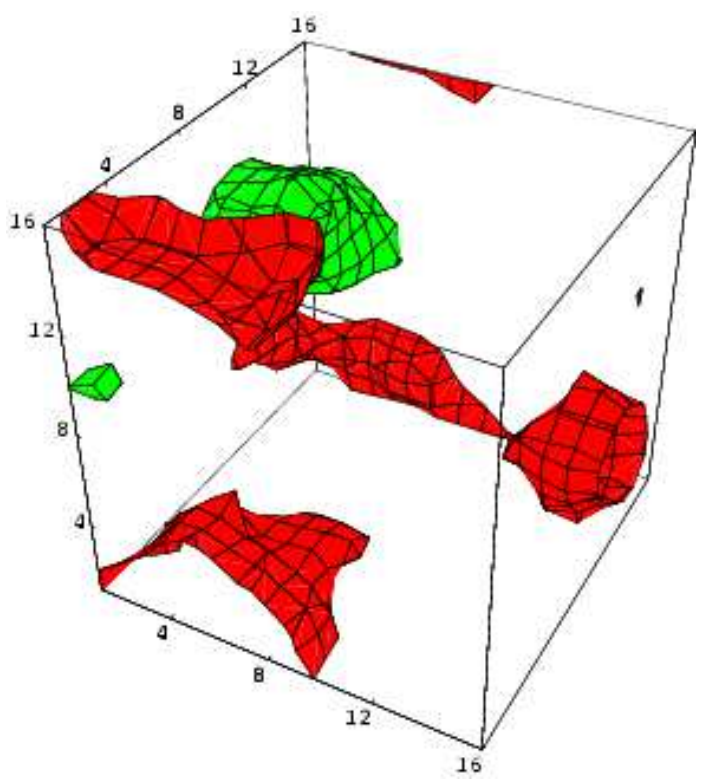

(a)

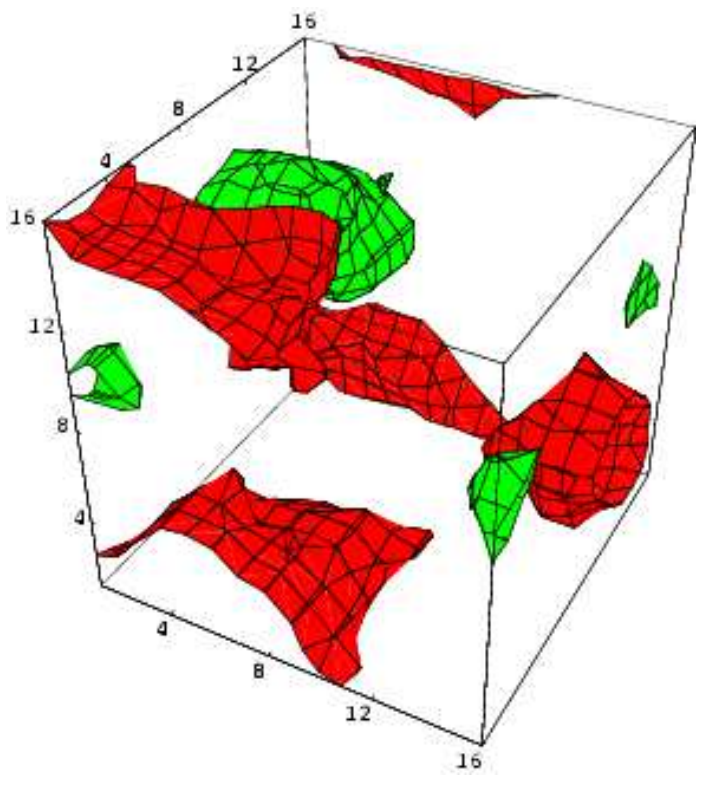

(c)

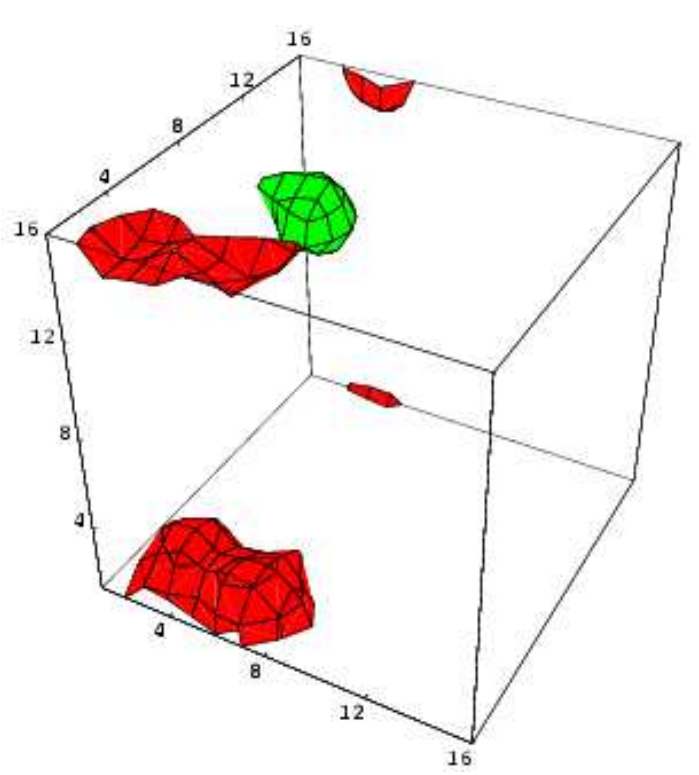

(b)

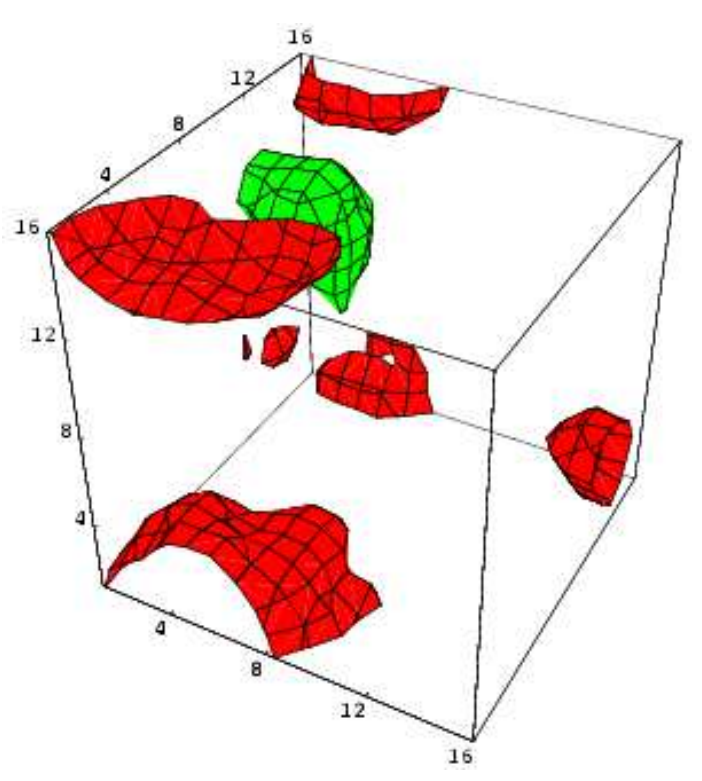

(d)

FIG. 27: Direct comparison between the isosurface plots with respect to $R$ (left: with $R_{\text {cut }}=0.99$ in (a) and $R_{\text {cut }}=0.98$ in (c)) and the isosurface plots with respect to the truncated topological density $q_{\lambda_{\text {cut }}}$ with $\lambda_{\text {cut }}=200 \mathrm{MeV}$ (9 pairs of non-zero modes) (right, with $q_{\text {cut }} / q_{\max }=0.2$ in (b) and $q_{\text {cut }} / q_{\max }=0.1$ in $\left.(\mathrm{d})\right)$. All subfigures show the same time slice $t=6$ of the typical $Q=0$ configuration of the $16^{3} \times 32$ ensemble at $\beta=8.45$. The similarity is found in all timeslices. 


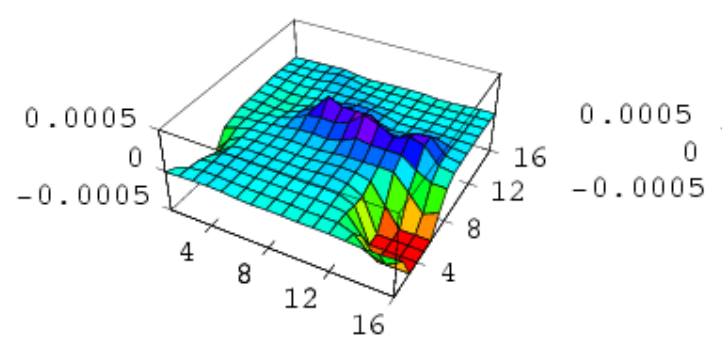

(a)

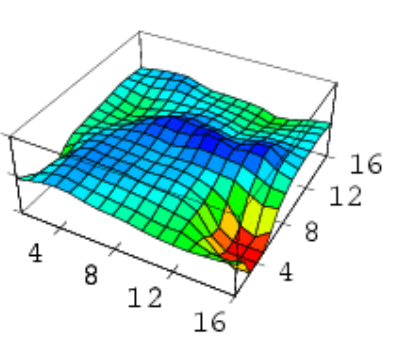

(b)

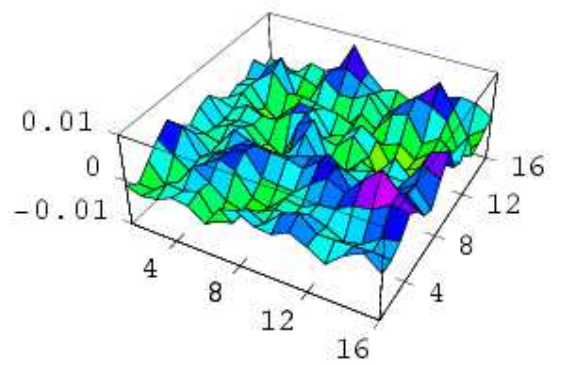

(c)

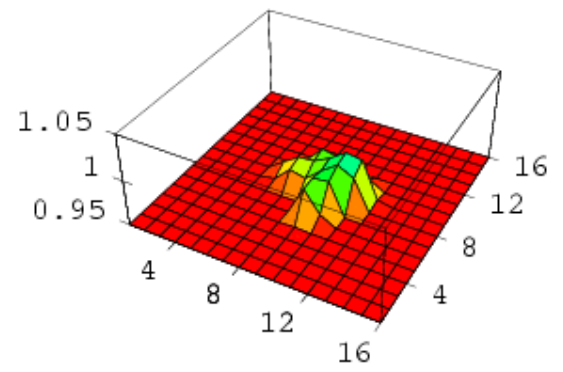

(d)

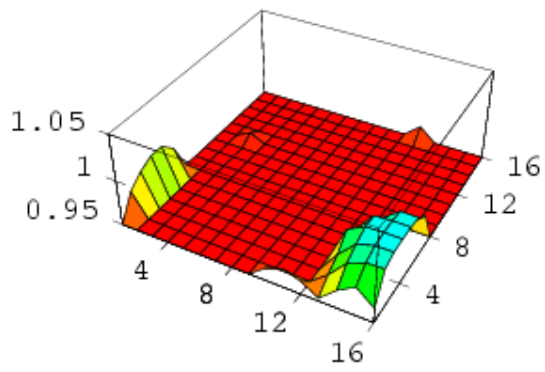

(e)

FIG. 28: Two-dimensional section through the typical $Q=0$ configuration generated at $\beta=8.45$ on a $16^{3} \times 32$ lattice. Direct comparison of $(\mathrm{a}) \tilde{q}_{\mathrm{IR}}(x)$ based on the infrared field strength tensor based on 10 non-zero modes (5 pairs), (b) the mode-truncated density $q_{\lambda_{\text {cut }}}(x)$ with $\lambda_{\text {cut }}=200 \mathrm{MeV}$ (for this configuration it amounts to 9 pairs of non-zero modes), (c) the all-scale charge density $q(x)$. Subpanels (d) and (e) show the positive and negative part of the local (anti-)selfduality variable $R(x)$. Note the 20-fold larger vertical scale in (c) compared to (b). The scale of (a) is freely adapted to that of (b).

non-percolating regime $\left(R_{\text {cut }}=0.99\right)$, with respect to charge volume, is illustrated in Fig. 26 for the $Q=0$ configuration already considered in Figs. 6] and 24, In order to define the cluster charge, the all-scale topological charge density $q(x)$ (18) has been summed over the sites belonging to the respective connected $R$-clusters.

Before percolation sets in (at $R_{\text {cut }}=0.99$ ) one finds in this particular configuration $O(20)$ significant clusters. Among them one cluster has an exceedingly large volume and two have a cluster charge $Q_{\text {cluster }}=1$ or -3 . Deep in the percolation regime (at $R_{\text {cut }}=0.96$ ), there are essentially only two significant $R$-clusters left over which share the total occupied volume 
in the ratio 55/45. Their cluster charges are large and must compensate each other. Even in this state, the distance between the two clusters is still large, $d\left(c_{1}, c_{2}\right) \approx 7 a$.

Near the maximum multiplicity, before the percolation sets in, for a suitable ultraviolet cut-off $\lambda_{\text {cut }}$ the number of $q$-clusters is of the same order as the number of (anti-)selfdual $R$-clusters (domains) defined with a corresponding number of modes. Moreover, it turns out, that the respective isosurface plots are closely correlated. We consider this coincidence as an argument for the importance of locally (anti-)selfdual infrared gauge fields as pert of the vacuum structure at a cut-off scale of $200 \mathrm{MeV}$. The correlation of positions and sizes of both types of clusters is shown in Fig. 27] where one timeslice is visualized by means of $R(x)$ on the left side and of $q_{\lambda_{\text {cut }}}(x)$ with $\lambda_{\text {cut }}=200 \mathrm{MeV}$ (which actually corresponds to 9 pairs of non-zero modes for this configuration) on the right side. The same kind of similarity exists in all timeslices.

Summarizing, in Fig. 28 we show the various ways to present the topological structure in a two-dimensional section. The upper panels present the profiles of various densities, $\tilde{q}_{\mathrm{IR}}(x)$ based on the infrared field strength, the mode-truncated density $q_{\lambda_{\text {cut }}}(x)$ and the all-scale density $q(x)$. The lower panels show how the positive and negative part of $R(x)$ restricted to the interval $0.95<|R(x)|<1$ highlights the regions of selfduality and antiselfduality. The plots illustrate this for the same $Q=0$ example configuration as visualized many times.

\section{DISCUSSION AND CONCLUSIONS}

We have reported on several attempts to elucidate the structure of the (quenched) QCD vacuum using overlap fermions as a probe. In particular, we have looked at the localization properties of the eigenmodes and of the topological charge density which is built up by all modes. Furthermore, we have studied the chiral properties of the eigenmodes of the overlap Dirac operator and tried to relate this to the topological charge density. Finally we have searched for (anti-)selfdual domains of the ultraviolet filtered field strength tensor.

As a side-result of this work, we have obtained the topological susceptibility for the ensembles in use (from the index of each configuration) and the average spectral density. The analysis of the latter has resulted in an effective chiral condensate $\Sigma_{\text {eff }}$ for each lattice size. The final analysis of this in the light of quenched chiral perturbation theory, the comparison 
with random matrix theory, in particular under the aspect of spectral correlations, will be subject of a separate publication. The emphasis of the present paper was put on the chiral and topological structural properties.

While average properties as the dimensionality of eigenmodes (for zero modes and nonzero modes in special intervals of eigenvalues $\lambda$ ) could be obtained by the scaling properties of the average inverse participation ratio (and its generalization based on higher moments of the scalar density) in agreement with critical spectral correlations (not described in this paper), the space-time structure of single modes and of the topological charge density could be studied by employing a cluster algorithm and, subsequently, a random walk algorithm. While the cluster algorithm locates and separates the clusters, depending on a cut ( $q_{\text {cut }}$ or $\left.p_{\text {cut }}\right)$ applied to the quantity that is being considered, the random walkers help to specify the space-time dimension of the cluster by means of their return probability to the cluster center from which they start.

A necessary condition for a pion to be able to propagate through a given lattice vacuum is that the scalar density of the modes forms percolating clusters, such that any two separate points on the lattice must be "connected" (in the sense defined in Section IE with non-zero measure when integrated over all configurations). Similar properties might be established for other quantities, like the topological charge density.

In the specific case of the topological charge density one finds at low enough density only two connected clusters of opposite charge, which are infinitely thin in the co-direction and form a multi-layered compound that covers all space-time. This result can hardly be reconciled with the picture of a dilute gas of semi-classical, finite-sized, in four dimensions coherently extended excitations (instantons or other classical solutions). Since the topological charge density correlator in the vacuum must be negative for all non-zero distances (on the lattice at least beyond a distance of $O(a)$ set by the non-ultralocal density definition in terms of the overlap operator), this requires a local fractal dimension $d^{*}<4$ for the topological density. The simultaneous presence of infrared (long-range) and ultraviolet $(O(a))$ features motivates to call the overlap topological density "all-scale density", as long as no mode-truncation is applied. This picture is known from the work of Horvath et al., but it does not exclude the presence of even lower dimensional structure inside the two clusters. Here we complete the picture by pointing out (see Table V in the Appendix) that percolating 


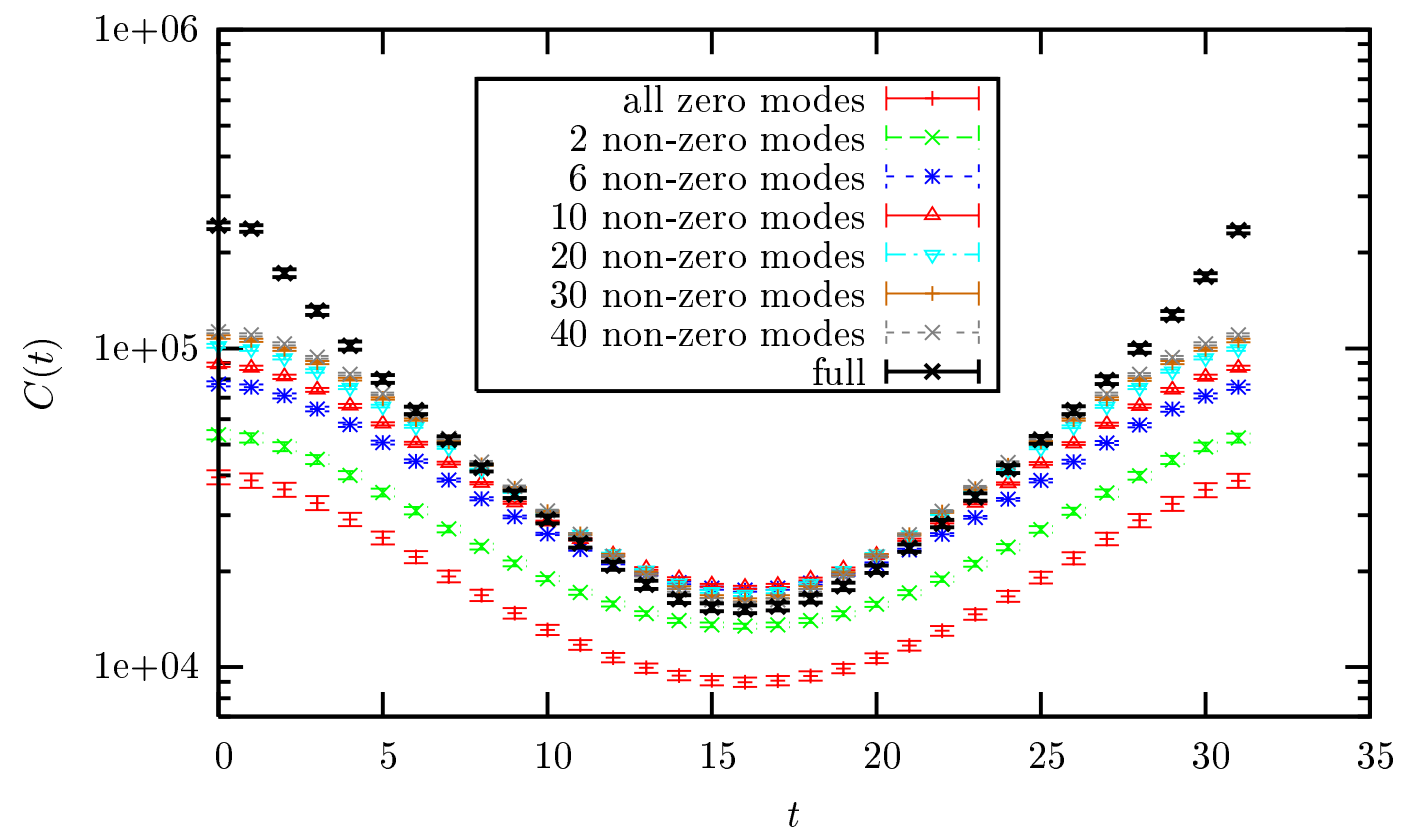

FIG. 29: The full pion propagator compared with the contribution of the zero modes alone and the cumulative approximation by various numbers of non-zero modes (see legend box) is shown in analogy to Fig. 1. For this calculation 250 configurations have been used from the $16^{3} \times 32$ lattice ensemble generated at $\beta=8.45$.

clusters of topological charge already appear with lower dimensions $d^{*}=1-2$ at higher $q_{\text {cut }} \approx 0.25 q_{\max }$.

Similar things are observed for single modes of the overlap operator. Percolation sets in at dimensions $d^{*} \approx 2$ and $p_{\text {cut }} \approx 0.20 p_{\max }$ for zero modes and the first few non-zero modes, whereas higher modes percolate already at higher $p_{\text {cut }} / p_{\max }$.

In the Introduction (in Fig. 1) we have seen that the long-distance part of the pion propagator is well saturated by the lowest 40 eigenmodes of the overlap operator. In more detail, Fig. 29] shows the pion correlator separately for an increasing number of modes, from the zero modes alone up to the additional inclusion of 40 non-zero modes.

In Fig. 30 we show the convergence to the full propagator separately for various time differences $t$ and in Fig. 31 the effective pion mass separately for the full propagator and different mode-truncated approximations to it, also as a function of $t$. We see that the pion propagates with approximately $70 \%$ of its physical mass even when the quark propagator 


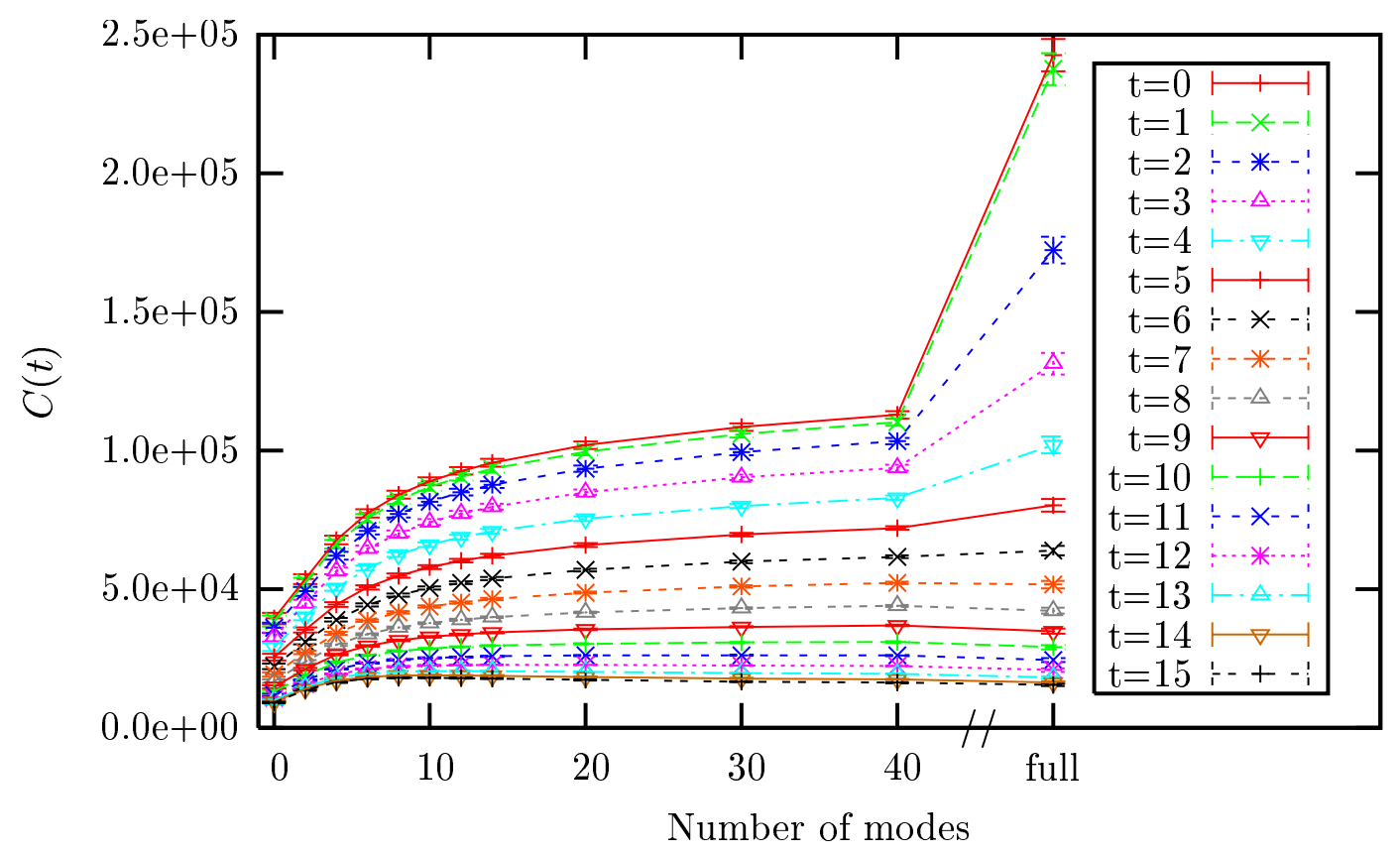

FIG. 30: The dependence of the pion propagator for various $t$ (see legend box) on the number of accumulated non-zero modes is presented for the $16^{3} \times 32$ lattice ensemble generated at $\beta=8.45$.

is stripped down to the zero modes.

If we wanted to define the mobility edge by the lowest mode at which propagation sets in, we would have to conclude that the mobility edge is at $\lambda=0$, and that the (quenched) $\mathrm{QCD}$ vacuum is critical in the whole lower part $(\lambda<200 \mathrm{MeV})$ of the spectrum, very analogous to certain 2D or 3D disordered condensed matter systems [87]. The level compressibility (not described in this paper) supports this analogy.

What changes in the vacuum picture if we would restrict our analysis of the vacuum structure to the lowest part of the spectrum? If the topological density is ultraviolet filtered by truncating its definition to the lowest modes, the emerging picture is completely different from what we have outlined above. The topological charge density correlator would now be resembling the presence of clusters of finite extent being subject to some weak but nontrivial intercluster correlations, increasing with the cut-off $\lambda_{\text {cut }}$. This picture is basically reproduced by the cluster analysis and the random walker algorithm.

In the Appendix we summarize our main results concerning the dimensionality of the all-scale and of the mode-truncated (ultraviolet filtered) topological charge density (Tables 


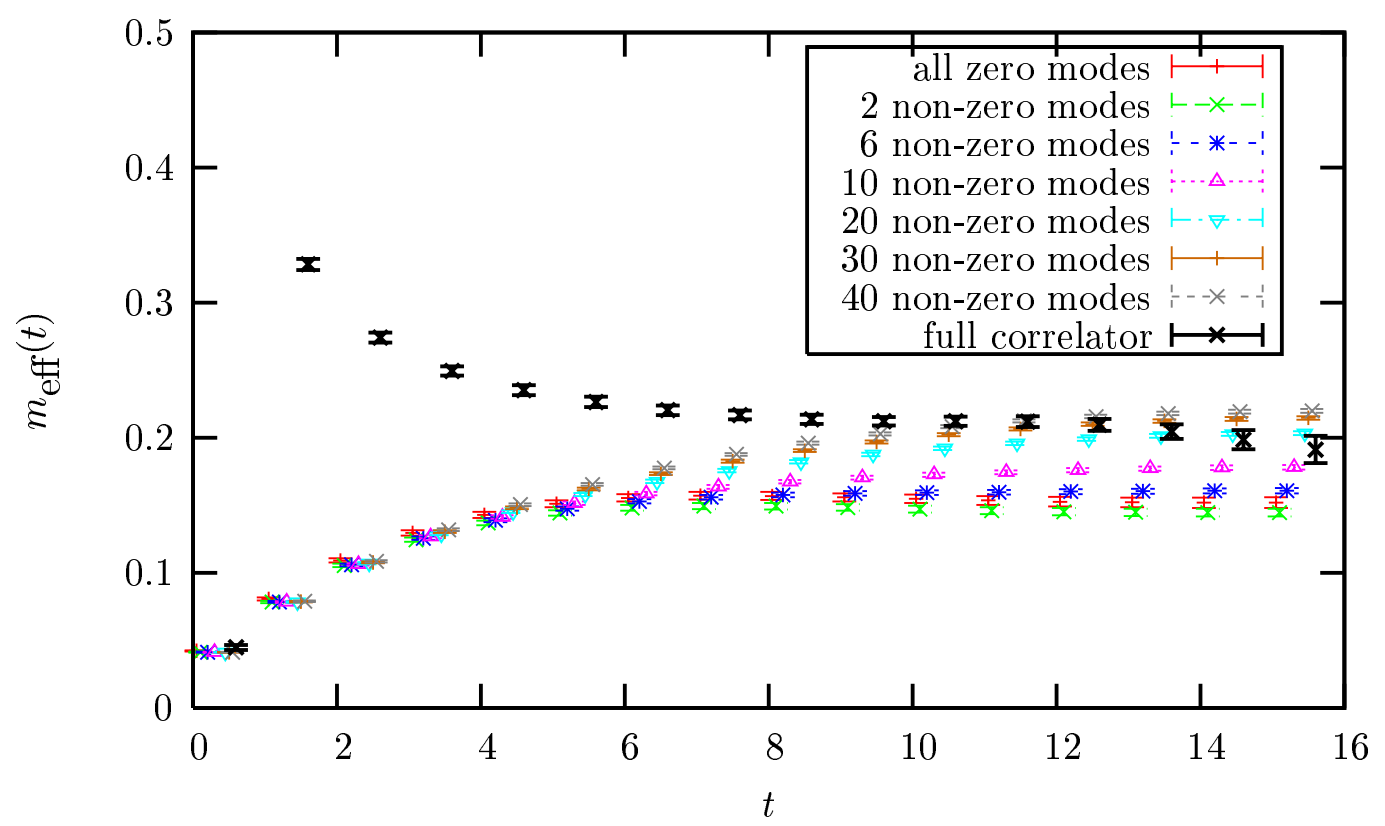

FIG. 31: The effective-mass plot vs. $t$ for the $16^{3} \times 32$ lattice ensemble generated at $\beta=8.45$. This is shown for the full pion propagator and for various numbers (see legend box) of non-zero modes included in the approximation.

$\mathrm{V}$ and VI) and of the scalar density of zero modes and selected non-zero modes (Tables VII and VIII). Here we also test the reliability of the "covering sphere method" introduced in subsection IVD, that attempts to estimate the effective dimensionality by the growth of the integrated quantity under consideration (the topological charge and scalar density) with the radius of a sphere covering a cluster. Although this latter method clearly defines a certain cut-off that separates a linear growth from a nonlinear one in both cases, the estimated dimensionalities do not coincide with the random walker method. This shows that the density inside the clusters is very inhomogeneous.

Concerning the all-scale topological density, it is remarkable that at low values of the density (at the lowest cut-off $q_{\text {cut }}$ defining the clusters) the dimensionality is practically independent of the lattice cut-off $a$ and becomes almost equal three. The covering sphere method finds it somewhat bigger than three. At higher values of the density smaller dimensionalities are found. There the covering sphere method gives an effective dimension equal to one, i.e. the charge inside a cluster grows linearly with the size. For the mode-truncated density at lowest values of the density (at a low cut-off defining the clusters) the clusters' 
dimensionality surprisingly is also close to three. How the infrared clusters (with a fixed $\left.\lambda_{\text {cut }}\right)$ start to percolate we can describe in the following way: at high values of the density they are well-separated and localized to zero dimensional balls or one dimensional rods of finite thickness, before they start to percolate at somewhat lower density with an effective dimension still relatively low, $d^{*}=1.5-2$. Finally, at even lower values of the density, they become extended in essentially three dimensions with an $a$-independent thickness of $O\left(\Lambda_{\mathrm{QCD}}\right)$.

A nonvanishing local chirality $X_{\lambda}(x)$ is assumed by individual lower-lying non-zero modes in regions of their preferential localization (i.e. at high scalar density $p(x)$ ). This effect could be demonstrated to be correlated to the ultraviolet filtered topological density $q_{\lambda}$ over a distance comparable to the correlation length of the density itself. Here we can only say that this ultraviolet filtered density is resulting from the correlated behavior of all lowestlying modes. On the other hand, this type of correlation of $X_{\lambda}$ with the topological charge density is a natural consequence in the instanton model where the topological charge is simply carried by the semiclassical background and the non-zero modes are superpositions of the (anti-)instantons' zero modes.

The all-scale topological density does not follow this pattern. It deserves its name at low cut-off $q_{\text {cut }}$ where it combines long-range coherence on one hand and lower-dimensional, i.e. singular structure on the other. When the cut-off is increased, percolation breaks down and an increasing number of isolated clusters appears with lower and lower dimensions. In this sense the all-scale topological density is multifractal, too. It should be noted that the maximal number of these clusters seems to depend only on the lattice size in lattice units. In a given physical volume it is diverging with the lattice spacing $a \rightarrow 0$.

Applying the same methods to the scalar density of individual modes (from zero modes up to the highest analyzed modes) we also have found that at very low cut-off $p_{\text {cut }}$ they do not extend isotropically but show a lower-dimensional structure. At the lowest cut-off the effective dimension ranges from two-dimensional for zero modes and the very first non-zero modes to three-dimensional for the modes up to the 120th non-zero mode. This means that all the modes that are sufficient to saturate the pion propagator share this property of lowdimensionality. This is in agreement with the critical level statistics (being "non-metallic" following the condensed-matter terminology). 
Summarizing we can state that at sufficiently low density $(q(x)$ or $p(x))$ we find consistently a dimensionality $d^{*} \approx 3$ to characterize most considered densities. The only exception are the very lowest modes which show a two-dimensional structure with respect to the growth of the integrated norm. This special feature is corroborating the average properties that have been concluded from the scaling behavior of the IPR.

It is remarkable that the mode truncated topological charge density percolates already at relatively low (volume) packing fraction, when its domains have a finite extension, forming coherent clusters of finite thickness $O\left(\Lambda_{\mathrm{CD}}\right)$. This is impossible for the all-scale topological density. This kind of clustering of the ultraviolet filtered topological charge is corroborated by the search for (anti-)selfdual domains, employing the ultraviolet filtered field strength tensor. The latter can be defined by means of the overlap operator, too, employing filtering in the form of truncating the spectral representation.

In conclusion: the vacuum picture exhibited by the modes that are sufficient to build up the pion propagator resembles a dilute liquid of separated (anti-)selfdual excitations if looked at it at higher cut-off $q_{\text {cut }}$ of the topological charge density and a dilute, percolating network at lower cut-off. The less-than-three dimensional pinning-down of the very lowest modes at very high scalar density $p(x)$ (and of the topological density at very high values of $\left.q_{\lambda}(x)\right)$ might be related to singular (confining) excitations like monopoles or vortices. This possibility is presently under investigation. In particular, the two-dimensional structure of the zero modes (with pointlike peaks on top of the latter) intriguingly suggests that they are pinned to vortex surfaces and vortex intersections [85]. In this sense we may conjecture that the lowest part of the fermion spectrum is both realizing chiral symmetry breaking and tracing the agents of confinement. This would be close in spirit to recent attempts in the literature to extract the dynamics of the Polyakov loop from the spectral properties of the Dirac operator [86, 88, 89].

It will be our next task to extend these studies to finite temperature and full QCD in order to disclose the changes of topological structure under the influence of dynamical quarks and those accompanying the chiral phase transition. 


\section{APPENDIX: TABLES OF DIMENSIONS}

\begin{tabular}{|c|cccccc|}
\hline$q_{\text {cut }} / q_{\text {max }}$ & 0.00 & 0.10 & 0.20 & 0.30 & 0.40 & 0.50 \\
\hline $12^{3} \times 24$ all-scale & $\mathbf{2 . 7 ( 1 )}$ & $\mathbf{1 . 5 ( 1 )}$ & $\mathbf{1 . 1}(\mathbf{1})$ & $0.7(1)$ & $0.4(1)$ & $0.1(1)$ \\
$12^{3} \times 24 \lambda_{\text {cut }} \leq 200 \mathrm{MeV}$ & $\mathbf{3 . 1}(\mathbf{1})$ & $\mathbf{1 . 8}(\mathbf{1})$ & $\mathbf{1 . 5}(\mathbf{1})$ & $1.0(1)$ & $0.7(1)$ & $0.2(1)$ \\
$12^{3} \times 24 \lambda_{\text {cut }} \leq 800 \mathrm{MeV}$ & $\mathbf{3 . 0 ( 1 )}$ & $\mathbf{1 . 8}(\mathbf{1})$ & $\mathbf{1 . 5}(\mathbf{1})$ & $\mathbf{1 . 0}(\mathbf{1})$ & $0.7(1)$ & $0.2(1)$ \\
\hline $16^{3} \times 32$ all-scale & $\mathbf{2 . 6 ( 2 )}$ & $\mathbf{1 . 5 ( 1 )}$ & $\mathbf{1 . 1}(\mathbf{1})$ & $0.9(1)$ & $0.5(1)$ & $0.1(1)$ \\
$16^{3} \times 32 \lambda_{\text {cut }} \leq 200 \mathrm{MeV}$ & $\mathbf{3 . 0 ( 1 )}$ & $\mathbf{2 . 1}(\mathbf{2})$ & $\mathbf{1 . 8}(\mathbf{1})$ & $1.2(1)$ & $0.8(1)$ & $0.3(1)$ \\
$16^{3} \times 32 \lambda_{\text {cut }} \leq 634 \mathrm{MeV}$ & $\mathbf{3 . 0 ( 1 )}$ & $\mathbf{1 . 9}(\mathbf{1})$ & $\mathbf{1 . 7}(\mathbf{1})$ & $1.2(1)$ & $0.7(1)$ & $0.3(1)$ \\
\hline
\end{tabular}

TABLE V: Effective dimension $d^{*}$ of the leading cluster of the topological charge density based on the random walker approach. Dimensions referring to the percolating regime are printed in bold face. The data is averaged over the $12^{3} \times 24\left(16^{3} \times 32\right)$ lattice ensemble at $\beta=8.10(\beta=8.45)$.

\begin{tabular}{|c|cccccc|}
\hline$q_{\text {cut }} / q \max$ & 0.00 & 0.10 & 0.20 & 0.30 & 0.40 & 0.50 \\
\hline $12^{3} \times 24$ all-scale & $\mathbf{3 . 3 ( 1 )}$ & $\mathbf{3 . 1}(\mathbf{1})$ & 1 & 1 & 1 & 1 \\
$12^{3} \times 24 \lambda_{\text {cut }} \leq 200 \mathrm{MeV}$ & $\mathbf{2 . 5 ( 2 )}$ & 1 & 1 & 1 & 1 & 1 \\
$12^{3} \times 24 \lambda_{\text {cut }} \leq 800 \mathrm{MeV}$ & $\mathbf{2 . 9 ( 1 )}$ & $\mathbf{1 . 8}(\mathbf{1})$ & 1 & 1 & 1 & 1 \\
\hline $16^{3} \times 32$ all-scale & $\mathbf{3 . 3 ( 1 )}$ & $\mathbf{3 . 3}(\mathbf{1})$ & $\mathbf{2 . 5 ( 1 )}$ & 1 & 1 & 1 \\
$16^{3} \times 32 \lambda_{\text {cut }} \leq 200 \mathrm{MeV}$ & $\mathbf{1 . 9 ( 1 )}$ & 1 & 1 & 1 & 1 & 1 \\
$16^{3} \times 32 \lambda_{\text {cut }} \leq 634 \mathrm{MeV}$ & $\mathbf{2 . 3 ( 1 )}$ & 1 & 1 & 1 & 1 & 1 \\
\hline
\end{tabular}

TABLE VI: Effective dimension of the leading cluster of the topological charge density based on the covering sphere method. The data is averaged over the $12^{3} \times 24\left(16^{3} \times 32\right)$ lattice ensemble at $\beta=8.10(\beta=8.45)$. 


\begin{tabular}{|c|c|c|c|c|c|}
\hline$p_{\text {cut }} / p_{\max }$ & 0.00 & 0.10 & 0.20 & 0.30 & 0.40 \\
\hline zeromodes & $3.6(2)$ & $2.8(2)$ & $2.0(2)$ & $1.4(1)$ & $1.1(1)$ \\
\hline 1st non-zero mode & $3.5(1)$ & $2.9(2)$ & $1.9(2)$ & $1.4(1)$ & $1.1(1)$ \\
\hline 10th non-zero mode & $3.6(2)$ & $3.0(2)$ & $2.2(2)$ & $1.6(1)$ & $1.1(1)$ \\
\hline 30 th non-zero mode & $3.5(1)$ & $3.2(2)$ & $2.6(1)$ & $2.0(1)$ & $1.4(1)$ \\
\hline 50th non-zero mode & $3.6(2)$ & $3.3(1)$ & $2.8(1)$ & $2.1(1)$ & $1.5(1)$ \\
\hline 70th non-zero mode & $3.6(2)$ & $3.4(1)$ & $2.9(1)$ & $2.2(1)$ & $1.6(1)$ \\
\hline 90th non-zero mode & $3.5(2)$ & $3.3(2)$ & $3.0(1)$ & $2.4(1)$ & $1.7(1)$ \\
\hline 120th non-zero mode & $3.6(2)$ & $3.4(2)$ & $3.1(1)$ & $2.5(1)$ & $1.8(1)$ \\
\hline
\end{tabular}

TABLE VII: Effective dimension $d^{*}$ of the leading cluster of the scalar density based on the random walker approach. The data is averaged over the $16^{3} \times 32$ lattice ensemble generated at $\beta=8.45$. Dimensions referring to the percolating regime are printed in bold face.

\begin{tabular}{|c|ccccc|}
\hline$p_{\text {cut }} / p_{\text {max }}$ & 0.00 & 0.10 & 0.20 & 0.30 & 0.40 \\
\hline zeromodes & $\mathbf{1 . 9}(\mathbf{1})$ & $\mathbf{1}$ & $\mathbf{1}$ & 1 & 1 \\
1st non-zero mode & $\mathbf{2 . 1}(\mathbf{1})$ & $\mathbf{1}$ & $\mathbf{1}$ & 1 & 1 \\
10th non-zero mode & $\mathbf{2 . 5 ( 1 )}$ & $\mathbf{1}$ & $\mathbf{1}$ & $\mathbf{1}$ & $\mathbf{1}$ \\
30th non-zero mode & $\mathbf{2 . 9 ( 1 )}$ & $\mathbf{2 . 3}(\mathbf{1})$ & $\mathbf{1}$ & $\mathbf{1}$ & $\mathbf{1}$ \\
50th non-zero mode & $\mathbf{2 . 9 ( 1 )}$ & $\mathbf{2 . 4 ( 1 )}$ & $\mathbf{1}$ & $\mathbf{1}$ & 1 \\
70th non-zero mode & $\mathbf{3 . 0 ( 1 )}$ & $\mathbf{2 . 7 ( 1 )}$ & $\mathbf{1 . 9}(\mathbf{1})$ & $\mathbf{1}$ & $\mathbf{1}$ \\
90th non-zero mode & $\mathbf{3 . 1}(\mathbf{1})$ & $\mathbf{2 . 6 ( 1 )}$ & $\mathbf{1 . 9}(\mathbf{1})$ & $\mathbf{1}$ & $\mathbf{1}$ \\
120th non-zero mode & $\mathbf{3 . 1}(\mathbf{1})$ & $\mathbf{2 . 9}(\mathbf{1})$ & $\mathbf{2 . 3 ( 1 )}$ & $\mathbf{1}$ & $\mathbf{1}$ \\
\hline
\end{tabular}

TABLE VIII: Effective dimension of the leading cluster of the scalar density based on the covering sphere method. The data is averaged over the $16^{3} \times 32$ lattice ensemble generated at $\beta=8.45$. 


\section{Acknowledgement}

The numerical calculations have been performed on the IBM p690 at HLRN (Berlin) and NIC (Jülich), as well as the PC farm at DESY (Zeuthen), the LRZ München and the Sektion Physik of LMU München. We thank all these institutions for technical support. This work has been financially supported in part by the EU Integrated Infrastructure Initiative Hadron Physics (I3HP) under contract RII3-CT-2004-506078 and by the DFG under contract FOR 465 (Forschergruppe Gitter-Hadronen-Phänomenologie). E.-M. I. is grateful to P. van Baal, F. Bruckmann, C. Gattringer, M. Müller-Preussker, A. Schäfer and S. Solbrig for inspiring discussions and collaboration on related projects. V. W. thanks the Yukawa Institute for Theoretical Physics at Kyoto University for the opportunity to participate in the YITP workshop on "Actions and symmetries in lattice gauge theory" (YITP-W-05-25) and to present part of the results. The discussions there were useful to complete this work. E.M. I. thanks the organizers of the Program INT-06-1 at the Institute for Nuclear Theory of the University of Washington, in particular E. Shuryak, for the opportunity to discuss these results, and the DoE for support of his participation.

[1] H. Neuberger, Phys. Lett. B417, 141 (1998), hep-lat/9707022.

[2] H. Neuberger, Phys. Lett. B427, 353 (1998), hep-lat/9801031.

[3] M. Lüscher, Phys. Lett. B428, 342 (1998), hep-lat/9802011.

[4] P. Hasenfratz, V. Laliena, and F. Niedermayer, Phys. Lett. B427, 125 (1998), heplat/9801021.

[5] F. Niedermayer, Nucl. Phys. Proc. Suppl. 73, 105 (1999), hep-lat/9810026.

[6] Y. Koma et al., PoS LAT2005, 300 (2006), hep-lat/0509164.

[7] E. M. Ilgenfritz et al., Nucl. Phys. Proc. Suppl. 153, 328 (2006), hep-lat/0512005.

[8] V. Weinberg et al., PoS LAT2006, 078 (2006), hep-lat/0610087.

[9] E. M. Ilgenfritz et al. (2006), hep-lat/0611007.

[10] V. Weinberg et al., PoS LAT2005, 171 (2006), hep-lat/0510056.

[11] T. A. DeGrand and A. Hasenfratz, Phys. Rev. D64, 034512 (2001), hep-lat/0012021.

[12] T. A. DeGrand, Phys. Rev. D64, 094508 (2001), hep-lat/0106001. 
[13] T. L. Ivanenko and J. W. Negele, Nucl. Phys. Proc. Suppl. 63, 504 (1998), hep-lat/9709130.

[14] H. Neff, N. Eicker, T. Lippert, J. W. Negele, and K. Schilling, Phys. Rev. D64, 114509 (2001), hep-lat/0106016.

[15] R. W. Haymaker, Phys. Rept. 315, 153 (1999), hep-lat/9809094.

[16] J. Greensite, Prog. Part. Nucl. Phys. 51, 1 (2003), hep-lat/0301023.

[17] A. Di Giacomo, Acta Phys. Polon. B36, 3723 (2005), hep-lat/0510065.

[18] C. Wetterich, AIP Conf. Proc. 739, 123 (2005), hep-ph/0410057.

[19] T. Schäfer and E. V. Shuryak, Rev. Mod. Phys. 70, 323 (1998), hep-ph/9610451.

[20] P. Gerhold, E. M. Ilgenfritz, and M. Müller-Preussker, Nucl. Phys. B760, 1 (2007), hep$\mathrm{ph} / 0607315$.

[21] E.-M. Ilgenfritz, M. L. Laursen, G. Schierholz, M. Müller-Preussker, and H. Schiller, Nucl. Phys. B268, 693 (1986).

[22] M. Teper, Phys. Lett. B171, 86 (1986).

[23] D. A. Smith and M. J. Teper (UKQCD), Phys. Rev. D58, 014505 (1998), hep-lat/9801008.

[24] M. Garcia Perez, O. Philipsen, and I.-O. Stamatescu, Nucl. Phys. B551, 293 (1999), heplat/9812006.

[25] T. A. DeGrand, A. Hasenfratz, and D.-c. Zhu, Nucl. Phys. B475, 321 (1996), hep-lat/9603015.

[26] T. A. DeGrand, A. Hasenfratz, and D.-c. Zhu, Nucl. Phys. B478, 349 (1996), hep-lat/9604018.

[27] M. Feurstein, E.-M. Ilgenfritz, M. Müller-Preussker, and S. Thurner, Nucl. Phys. B511, 421 (1998), hep-lat/9611024.

[28] T. A. DeGrand, A. Hasenfratz, and T. G. Kovacs, Nucl. Phys. B505, 417 (1997), heplat/9705009.

[29] T. A. DeGrand, A. Hasenfratz, and T. G. Kovacs, Nucl. Phys. B520, 301 (1998), heplat/9711032.

[30] J. W. Negele, Nucl. Phys. Proc. Suppl. 73, 92 (1999), hep-lat/9810053.

[31] I. Horvath et al., Phys. Rev. D67, 011501 (2003), hep-lat/0203027.

[32] S. O. Bilson-Thompson, D. B. Leinweber, and A. G. Williams, Ann. Phys. 304, 1 (2003), hep-lat/0203008.

[33] I. Horvath et al., Phys. Rev. D68, 114505 (2003), hep-lat/0302009.

[34] I. Horvath et al., Phys. Lett. B612, 21 (2005), hep-lat/0501025.

[35] E. Witten, Nucl. Phys. B149, 285 (1979). 
[36] I. Horvath, N. Isgur, J. McCune, and H. B. Thacker, Phys. Rev. D65, 014502 (2002), heplat/0102003.

[37] I. Horvath et al., Phys. Rev. D66, 034501 (2002), hep-lat/0201008.

[38] C. Gattringer, Phys. Rev. Lett. 88, 221601 (2002), hep-lat/0202002.

[39] K. F. Liu (2006), hep-lat/0609033.

[40] K. F. Liu, A. Alexandru, and I. Horvath (2007), hep-lat/0703010.

[41] T. A. DeGrand and S. Schaefer, Comput. Phys. Commun. 159, 185 (2004), hep-lat/0401011.

[42] L. Giusti and S. Necco, PoS LAT2005, 132 (2006), hep-lat/0510011.

[43] D. Galletly et al. (2006), hep-lat/0607024.

[44] C. Aubin et al. (MILC), Nucl. Phys. Proc. Suppl. 140, 626 (2005), hep-lat/0410024.

[45] C. Bernard et al., PoS LAT2005, 299 (2006), hep-lat/0510025.

[46] F. V. Gubarev, S. M. Morozov, M. I. Polikarpov, and V. I. Zakharov, JETP Lett. 82, 343 (2005), hep-lat/0505016.

[47] M. I. Polikarpov, F. V. Gubarev, S. M. Morozov, and V. I. Zakharov, PoS LAT2005, 143 (2006), hep-lat/0510098.

[48] M. I. Polikarpov, F. V. Gubarev, S. M. Morozov, and S. N. Syritsyn, Nucl. Phys. Proc. Suppl. 153, $221(2006)$.

[49] T. A. DeGrand and A. Hasenfratz, Phys. Rev. D65, 014503 (2002), hep-lat/0103002.

[50] R. G. Edwards and U. M. Heller, Phys. Rev. D65, 014505 (2002), hep-lat/0105004.

[51] P. de Forcrand (2006), hep-lat/0611034.

[52] D. J. R. Pugh and M. Teper, Phys. Lett. B224, 159 (1989).

[53] V. I. Zakharov, Phys. Atom. Nucl. 68, 573 (2005), hep-ph/0410034.

[54] V. I. Zakharov (2006), hep-ph/0602141.

[55] V. I. Zakharov (2006), hep-ph/0612341.

[56] V. I. Zakharov (2006), hep-ph/0612342.

[57] A. M. Garcia-Garcia and J. C. Osborn, PoS LAT2005, 265 (2006), hep-lat/0509118.

[58] A. M. Garcia-Garcia and J. C. Osborn, Nucl. Phys. A770, 141 (2006), hep-lat/0512025.

[59] A. M. Garcia-Garcia and J. C. Osborn (2006), hep-lat/0611019.

[60] P. H. Damgaard, Nucl. Phys. B608, 162 (2001), hep-lat/0105010.

[61] I. Horvath et al., Phys. Lett. B617, 49 (2005), hep-lat/0504005.

[62] M. Göckeler, A. S. Kronfeld, M. L. Laursen, G. Schierholz, and U. J. Wiese, Phys. Lett. B233, 
$192(1989)$.

[63] M. Lüscher and P. Weisz, Commun. Math. Phys. 97, 59 (1985).

[64] M. Lüscher and P. Weisz, Phys. Lett. B158, 250 (1985).

[65] G. P. Lepage and P. B. Mackenzie, Phys. Rev. D48, 2250 (1993), hep-lat/9209022.

[66] M. G. Alford, W. Dimm, G. P. Lepage, G. Hockney, and P. B. Mackenzie, Phys. Lett. B361, 87 (1995), hep-lat/9507010.

[67] J. Snippe, Nucl. Phys. B498, 347 (1997), hep-lat/9701002.

[68] C. Gattringer, R. Hoffmann, and S. Schaefer, Phys. Rev. D65, 094503 (2002), heplat/0112024.

[69] S. Capitani, M. Göckeler, R. Horsley, P. E. L. Rakow, and G. Schierholz, Phys. Lett. B468, 150 (1999), hep-lat/9908029.

[70] P. Hernandez, K. Jansen, and M. Lüscher, Nucl. Phys. B552, 363 (1999), hep-lat/9808010.

[71] L. Giusti, C. Hoelbling, M. Lüscher, and H. Wittig, Comput. Phys. Commun. 153, 31 (2003), hep-lat/0212012.

[72] T.-W. Chiu, C.-W. Wang, and S. V. Zenkin, Phys. Lett. B438, 321 (1998), hep-lat/9806031.

[73] T.-W. Chiu and S. V. Zenkin, Phys. Rev. D59, 074501 (1999), hep-lat/9806019.

[74] L. Del Debbio, L. Giusti, and C. Pica, Phys. Rev. Lett. 94, 032003 (2005), hep-th/0407052.

[75] T. Banks and A. Casher, Nucl. Phys. B169, 103 (1980).

[76] T. Wilke, T. Guhr, and T. Wettig, Phys. Rev. D57, 6486 (1998), hep-th/9711057.

[77] V. E. Kravtsov and K. A. Muttalib, Phys. Rev. Lett. 79, 1913 (1997), cond-mat/9703167.

[78] V. E. Kravtsov, cond-mat/9603166.

[79] D. Diakonov and V. Y. Petrov, Nucl. Phys. B272, 457 (1986).

[80] J. Greensite, S. Olejnik, M. Polikarpov, S. Syritsyn, and V. Zakharov, Phys. Rev. D71, 114507 (2005), hep-lat/0504008.

[81] E. Seiler, Phys. Lett. B525, 355 (2002), hep-th/0111125.

[82] A. Hasenfratz, Phys. Lett. B476, 188 (2000), hep-lat/9912053.

[83] E. M. Ilgenfritz and P. de Forcrand (unpublished).

[84] C. Gattringer, I. Hip, and C. B. Lang, Nucl. Phys. B597, 451 (2001), hep-lat/0007042.

[85] J. Gattnar et al., Nucl. Phys. B716, 105 (2005), hep-lat/0412032.

[86] C. Gattringer, Phys. Rev. Lett. 97, 032003 (2006), hep-lat/0605018.

[87] P. Markos, acta phys. slovaca 56, 561 (2006), cond-mat/0609580. 
[88] F. Bruckmann, C. Gattringer, and C. Hagen, Phys. Lett. B647, 56 (2007), hep-lat/0612020.

[89] F. Synatschke, A. Wipf, and C. Wozar (2007), hep-lat/0703018. 